ESAIM: M2AN 50 (2016) 43-75

DOI: $10.1051 / \mathrm{m} 2 \mathrm{an} / 2015030$
ESAIM: Mathematical Modelling and Numerical Analysis

www.esaim-m2an.org

\title{
EFFECTIVE TRANSMISSION CONDITIONS FOR THIN-LAYER TRANSMISSION PROBLEMS IN ELASTODYNAMICS. THE CASE OF A PLANAR LAYER MODEL
}

\author{
Marc Bonnet $^{1}$, Aliénor Burel ${ }^{1,2}$, Marc Duruflé $^{3}$ and Patrick Joly ${ }^{1}$
}

\begin{abstract}
This article is concerned with the design, analysis, numerical approximation and implementation of effective transmission conditions (ETCs) for the propagation of elastic waves through a thin planar elastic layer with small uniform thickness $\eta$ which is embedded in a reference elastic medium, under transient conditions, with both materials assumed to have isotropic properties. A family of ETCs of order $k$ (i.e. whose approximation error is of expected order $O\left(\eta^{k+1}\right)$ ) is formulated by deriving and exploiting a formal asymptotic expansion in powers of $\eta$ of the transmission solution inside the layer. The second-order ETCs are then retained as the main focus for the remainder of the article, and given a full justification in terms of both the stability of the resulting transient elastodynamic problem and the error analysis. The latter is performed by establishing and justifying asymptotic expansions for the solutions of both the exact transmission problem and its approximation based on the second-order ETCs. As a result, the error (in energy norm) between those two solutions is shown to be, as expected, of order $O\left(\eta^{3}\right)$. Finally, the numerical approximation of the proposed second-order ETC within the framework of spectral element methods is studied, with special attention devoted to the selection of a robust time-stepping scheme that is mostly explicit (and conditionally stable). Among these, a scheme that is implicit only for the interfacial degrees of freedom, termed semi-implicit, is shown to be stable under the same stability condition as for the layer-less configuration. The main theoretical results of this work are illustrated and validated by $2 \mathrm{D}$ and $3 \mathrm{D}$ numerical experiments under transient elastodynamic conditions.
\end{abstract}

Mathematics Subject Classification. 35L05, 35C20, 74B05, 65N12, 65N30.

Received May 27, 2014. Revised February 10, 2015.

Published online November 9, 2015.

\section{INTRODUCTION}

This article is concerned with the design, analysis, numerical approximation and implementation of effective transmission conditions for the propagation of elastic waves through a thin planar elastic layer with uniform thickness $\eta$. As we consider situations where $\eta$ may be much smaller than a characteristic wavelength associated

\footnotetext{
Keywords and phrases. Thin layer approximations, elastodynamics, transmission, asymptotic expansion.

1 POEMS (ENSTA ParisTech, CNRS, INRIA, Université Paris-Saclay), Palaiseau, France.

mbonnet@ensta.fr; patrick.joly@inria.fr

2 Laboratoire de Mathématiques d'Orsay, Université Paris-Sud, Orsay, France. alienor.burel@gmail.com

3 MAGIQUE 3D team, INRIA Bordeaux Sud-Ouest, Talence, France. marc.durufle@inria.fr
} 
with the source or initial conditions, numerical methods applied to this problem are penalized by the computational constraints (in terms of the mesh size, and consequently of the critical time step $\Delta t_{c}$ for conditionallystable time stepping schemes) induced by the need to geometrically resolve the thin layer. An idea for avoiding these constraints is to replace the exact transmission conditions by (approximate) effective transmission conditions (ETCs) that connect the solution on the two sides of the layer boundary, thereby avoiding the need to mesh the layer and solve the exact transmission problem. The main expected computational benefit lies in preventing $\Delta t_{c}$ from becoming very small in the thin-layer limit. Our results will show that this objective is achieved in practice, with $\Delta t_{c}$ essentially independent of $\eta$.

The problem of formulating approximate models for configurations involving thin layers is far from new, especially when considered in connection with wave propagation. In mathematical terms, such situations involve the asymptotic analysis of a family of partial differential equations that depend on the small parameter $\eta$. Beginning in the early nineties, several authors considered (acoustic or electromagnetic) wave propagation problems involving media coated with a thin layer made of a different material, their goal being to approximate the exact transmission problem by means of equivalent boundary conditions in order to simplify the modeling and numerical solution. Early studies addressed the case of homogeneous thin layers under time-harmonic conditions [3,10]; later, periodic thin layers involving a spatial period proportional to $\eta$ (known as rough surfaces) have also been considered [1,8]. Finally, the case of an acoustic layer surrounding an elastic body has recently been addressed [20]. The natural generalization to transient problems involving thin layers then presents additional difficulties related to stability in time, which have been addressed in e.g. [14], where nonlinear materials are also considered. For the case of homogeneous layers, the de facto reference methodology of analysis exploits a rescaling of the through-thickness coordinate and an ansatz for the solution expressed as an expansion in powers of $\eta$ (with the exception of [10]). The case of periodic layers is a bit more difficult, and requires combining the foregoing approaches for thin layers with homogenization methods; this entails using more sophisticated asymptotic techniques such as multiscale expansions [7] or matched asymptotic expansions [18]. These studies lead in all cases to the formulation of generalized impedance boundary conditions (GIBCs), which are widely used in the engineering community [22,23]. Similar conditions also arise in connection with the scattering of waves by highly conducting obstacles $[2,16]$, with the thin-layer effect replaced by a skin effect.

When the thin layer is embedded within the reference medium, asymptotic methods lead to effective transmission conditions (ETCs) instead of GIBCs. Fewer investigations have so far been devoted to such situations. Transient electromagnetic problems involving homogeneous layers are considered in [5], while the transmission of time-harmonic acoustic or electromagnetic waves through rough heterogeneous interfaces is treated in [8]. ETCs for a thin conducting sheet is considered in connection with the eddy current model in [21]. Transmission conditions for linear elastic media are investigated in [12] under equilibrium (i.e. time-independent) conditions. An asymptotic model based on integral equations is proposed in [21] for the case of a thin conducting sheet embedded in a non-conducting space. Generally speaking, the consideration of embedded layers turns out to be technically more difficult than that of superficial layers, in particular regarding the error analysis in the limit $\eta \rightarrow 0$. It also leads to more complicated equivalent conditions, and raise stability issues.

To our best knowledge, asymptotic models for either time-harmonic or transient problems involving elastic thin layers embedded in elastic media have not been studied so far. This work, which draws inspiration from [5], purports to bridge this gap. Unlike in [5], we restrict ourselves to the case of a planar layer. We however propose a complete study within this setting, by establishing theoretical results on error analysis and stability, studying the stability conditions for several time-stepping schemes of a mostly-explicit nature, and performing several numerical experiments on 2D and 3D configurations under transient conditions.

The article is organized as follows. Section 2 sets the stage for the development of approximate layer models based on ETCs and states the main results of this work, namely the formulation of the second-order ETC and its justification in terms of stability in time (Thm. 2.3) and approximation order relative to the exact transmission problem (Thm. 2.5). The developments underlying these results are given next in Sections 3 and 4. ETCs of arbitrary order are first formulated in Section 3, on the basis of a formal asymptotic expansion of the displacement inside the layer in powers of $\eta$ and the analytic derivation of the relationships between 


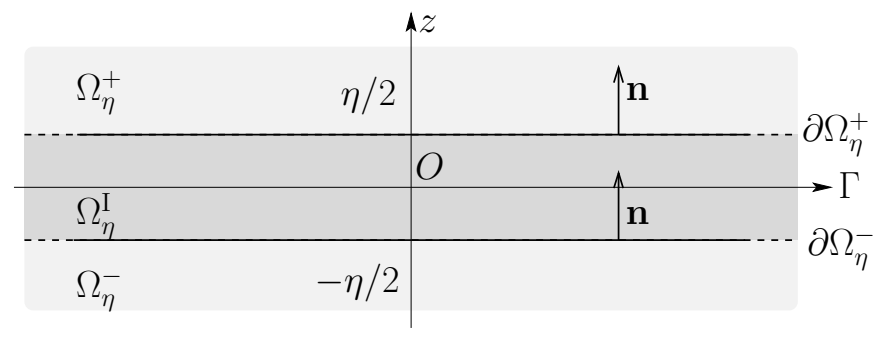

Figure 1. Problem setting.

corresponding expansions of displacements and tractions on the layer boundary. The second-order ETC is then retained as the main focus for the remainder of the article. Asymptotic expansions of both the exact transmission solution and its ETC-based approximation are defined and justified in Section 4, allowing to prove Theorem 2.5. Finally, Section 5 addresses the numerical approximation of the proposed second-order ETC, the selection of a robust time-stepping scheme and the validation of our main results through numerical experiments.

\section{Problem Setting AND StATEMENt of MAin Results}

\subsection{The elastodynamic transmission problem}

Let $d=2,3$. We consider a thin layer of thickness $\eta>0$ of an elastic material occupying the strip

$$
\left.\Omega_{\eta}^{\mathrm{I}}:=\mathbb{R}^{d-1} \times\right]-\eta / 2, \eta / 2\left[\subset \mathbb{R}^{d} .\right.
$$

The remaining portion of the propagation domain, assumed for simplicity to be unbounded, is thus

$$
\left.\Omega_{\eta}^{\mathrm{E}}:=\mathbb{R}^{d} \backslash \overline{\Omega_{\eta}^{\mathrm{I}}}=\Omega_{\eta}^{+} \cup \Omega_{\eta}^{-} \quad \text { with } \Omega_{\eta}^{+}:=\mathbb{R}^{d-1} \times\right] \eta / 2 ;+\infty\left[\text { and } \Omega_{\eta}^{-}:=\mathbb{R}^{d-1} \times\right]-\infty ;-\eta / 2,
$$

i.e. $\Omega_{\eta}^{+}$and $\Omega_{\eta}^{-}$are the regions situated above and below the thin layer, respectively. In addition,

$$
\partial \Omega_{\eta}^{+}=\mathbb{R}^{d-1} \times\{\eta / 2\} \text { and } \partial \Omega_{\eta}^{-}=\mathbb{R}^{d-1} \times\{-\eta / 2\}
$$

are the upper and lower boundaries of the layer, respectively connected to $\Omega_{\eta}^{+}$and $\Omega_{\eta}^{-}$, while $\Gamma:=\frac{1}{2}\left(\partial \Omega_{\eta}^{+}+\partial \Omega_{\eta}^{-}\right)$ is the parallel interface located halfway between the two boundaries (Fig. 1). Of course, $\partial \Omega_{\eta}^{+}$and $\partial \Omega_{\eta}^{-}$can be, in an obvious way, identified to $\Gamma$, which will be done implicitly several times in the sequel.

The material occupying the unbounded region $\Omega_{\eta}^{\mathrm{E}}$ is characterized by its (possibly heterogeneous) density $\rho$ and Lamé coefficients $\lambda, \mu$ in $\Omega_{\eta}^{\mathrm{E}}$.

The material inside the layer $\Omega_{\eta}^{\mathrm{I}}$ is assumed to be isotropic and homogeneous, i.e. to be characterized by constant material parameters $\rho^{\mathrm{I}}, \lambda^{\mathrm{I}}$ and $\mu^{\mathrm{I}}$. Possible extensions of this setting, which are not addressed in this article, include layers that are either non-planar, anisotropic, heterogeneous or of non-constant thickness.

To give meaning to asymptotic analyses with respect to $\eta$, we have to consider a family of problems. For these problems be well defined, we shall assume that $\rho, \lambda, \mu$ are given functions in $\mathbb{R}^{d}$ satisfying the usual requirements

$$
0<\rho_{-} \leq \rho(x) \leq \rho_{+}<+\infty, \quad 0<\lambda_{-} \leq \lambda(x) \leq \lambda_{+}<+\infty, \quad 0<\mu_{-} \leq \mu(x) \leq \mu_{+}<+\infty, \text { a.e. in } \mathbb{R}^{d},
$$

and that the mass density $\rho$ and Lamé coefficients $\lambda, \mu$ in the exterior domain $\Omega_{\eta}^{\mathrm{E}}$ are the restrictions to $\Omega_{\eta}^{\mathrm{E}}$ of these functions in $\mathbb{R}^{d}$ still denoted $\rho, \lambda, \mu$. In other words, the propagation media we consider are $\eta$-dependent perturbations of a fixed (i.e. independent of $\eta$ ) reference medium with $\rho, \lambda, \mu$ defined in the whole space $\mathbb{R}^{d}$. 
We assume for the sake of definiteness that the only excitation undergone by the system results from a given initial disturbance (i.e. known nonzero initial displacement $\mathbf{u}^{0}$ and velocity $\mathbf{u}^{1}$ ). Moreover, the support $D$ of the disturbance $\mathbf{u}^{0}, \mathbf{u}^{1}$ is compact and does not intersect the interface $\Gamma$, namely there exists $\eta_{0}$ such that $D \Subset \Omega_{\eta}^{\mathrm{E}}$ for any $\eta \leq \eta_{0}$. Other useful assumptions on $\mathbf{u}^{0}, \mathbf{u}^{1}$ and $\rho, \lambda, \mu$ will be made precise in Section 2.3.

Remark 2.1. The developments to follow could easily be adapted to other types of excitations (e.g. body forces) provided their support $D$ still meets the condition $D \Subset \Omega_{\eta}^{\mathrm{E}}$ for any $\eta \leq \eta_{0}$.

As a consequence of the foregoing assumptions, the elastic displacement fields $\mathbf{u}_{\eta}^{\mathrm{I}}(\mathbf{x}, t) \in \mathbb{R}^{d}$ in the layer $\left(\mathbf{x} \in \Omega_{\eta}^{\mathrm{I}}\right)$ and $\mathbf{u}_{\eta}^{\mathrm{E}}(\mathbf{x}, t) \in \mathbb{R}^{d}$ outside the layer $\left(\mathbf{x} \in \Omega_{\eta}^{\mathrm{E}}\right)$ satisfy the following elastodynamic equations and initial conditions:

$$
\begin{gathered}
\begin{cases}\rho^{\mathrm{I}} \partial_{t}^{2} \mathbf{u}_{\eta}^{\mathrm{I}}-\operatorname{div} \boldsymbol{\sigma}^{\mathrm{I}}\left(\mathbf{u}_{\eta}^{\mathrm{I}}\right)=0, & \text { in } \Omega_{\eta}^{\mathrm{I}} \times \mathbb{R}^{+}, \\
\mathbf{u}_{\eta}^{\mathrm{I}}(\cdot, 0)=0 \text { and } \partial_{t} \mathbf{u}_{\eta}^{\mathrm{I}}(\cdot, 0)=0, & \text { in } \Omega_{\eta}^{\mathrm{I}},\end{cases} \\
\begin{cases}\rho \partial_{t}^{2} \mathbf{u}_{\eta}^{\mathrm{E}}-\operatorname{div} \boldsymbol{\sigma}\left(\mathbf{u}_{\eta}^{\mathrm{E}}\right)=0, & \text { in } \Omega_{\eta}^{\mathrm{E}} \times \mathbb{R}^{+}, \\
\mathbf{u}_{\eta}^{\mathrm{E}}(\cdot, 0)=\mathbf{u}^{0} \text { and } \partial_{t} \mathbf{u}_{\eta}^{\mathrm{E}}(\cdot, 0)=\mathbf{u}^{1}, & \text { in } \Omega_{\eta}^{\mathrm{E}},\end{cases}
\end{gathered}
$$

where $\boldsymbol{\sigma}(\mathbf{u})$ and $\boldsymbol{\sigma}^{\mathrm{I}}(\mathbf{u})$ denote the stress tensor in the surrounding and layer material, respectively, given by Hooke's law applied to a given displacement $\mathbf{u}$ :

$$
\text { (a) } \boldsymbol{\sigma}^{\mathrm{I}}(\mathbf{u})=\lambda^{\mathrm{I}} \operatorname{Tr} \boldsymbol{\varepsilon}(\mathbf{u}) \mathrm{I}_{d}+2 \mu^{\mathrm{I}} \boldsymbol{\varepsilon}(\mathbf{u}), \quad \text { (b) } \boldsymbol{\sigma}(\mathbf{u})=\lambda \operatorname{Tr} \boldsymbol{\varepsilon}(\mathbf{u}) \mathrm{I}_{d}+2 \mu \boldsymbol{\varepsilon}(\mathbf{u}) .
$$

Here $\varepsilon(\mathbf{u})=\left(\nabla \mathbf{u}+\nabla^{T} \mathbf{u}\right) / 2$ is the linearized strain tensor, where $\nabla$ denotes the (matrix) gradient operator of vector fields. To any $\mathbf{x}$ in the layer $\Omega_{\eta}^{\mathrm{I}}$, we associate the local parametric coordinates $\left.(\mathbf{y}, \nu) \in \Gamma \times\right]-\eta / 2, \eta / 2[$ through

$$
\mathbf{x}=\mathbf{y}+\nu \mathbf{n}, \quad \mathbf{x} \in \Omega_{\eta}^{\mathrm{I}},
$$

where $\mathbf{n}$ is the unit normal vector to $\Gamma$ directed towards $\Omega_{\eta}^{+}$(see Fig. 1). Assuming the layer and exterior media to be perfectly bonded, equations (2.2) and (2.3) are coupled with transmission conditions on the interfaces $\partial \Omega_{\eta}^{\mathrm{E}}$ : for every $\mathbf{y}$ in $\Gamma$, we have

$$
\mathbf{u}_{\eta}^{\mathrm{E}}(\mathbf{y}, \pm \eta / 2, t)=\mathbf{u}_{\eta}^{\mathrm{I}}(\mathbf{y}, \pm \eta / 2, t), \quad \mathbf{t}\left(\mathbf{u}_{\eta}^{\mathrm{E}}\right)(\mathbf{y}, \pm \eta / 2, t)=\mathbf{t}^{\mathrm{I}}\left(\mathbf{u}_{\eta}^{\mathrm{I}}\right)(\mathbf{y}, \pm \eta / 2, t), \quad(\mathbf{y}, t) \in \Gamma \times \mathbb{R}^{+},
$$

where $\mathbf{t}(\mathbf{u}):=\boldsymbol{\sigma}(\mathbf{u}) \mathbf{n}$ and $\mathbf{t}^{\mathrm{I}}(\mathbf{u}):=\boldsymbol{\sigma}^{\mathrm{I}}(\mathbf{u}) \mathbf{n}$ are the traction vectors relative to $\Omega_{\eta}^{\mathrm{E}}$ and $\Omega_{\eta}^{\mathrm{I}}$.

The problem constituted by (2.2)-(2.4) and (2.6) is the exact transmission problem.

\subsection{Approximation via effective transmission conditions}

As stated in the introduction, the main objective of this work is to replace the exact transmission conditions (2.6) by (approximate) effective transmission conditions (ETCs) that connect the displacement $\mathbf{u}_{\eta}^{+}$on $\partial \Omega_{\eta}^{+}$with the displacement $\mathbf{u}_{\eta}^{-}$on $\partial \Omega_{\eta}^{-}$, without requiring the computation of the exact solution of $(2.2)-(2.4)$ and (2.6), but in such a way that the exact transmission conditions (2.6) are approximated within an error that vanishes in the limit $\eta \rightarrow 0$. To formulate and analyse ETCs, it is convenient to define, for any scalar or vector function $f$ admitting traces on the layer boundary $\partial \Omega_{\eta}^{ \pm}$, its generalized jump $\{f\}_{\eta}$ by setting

$$
\begin{aligned}
& \{f\}_{\eta}(\mathbf{y}, t)=\left([f]_{\eta}(\mathbf{y}, t),\langle f\rangle_{\eta}(\mathbf{y}, t)\right), \quad(\mathbf{y}, t) \in \Gamma \times \mathbb{R}^{+} \\
& \quad \text { with } \quad[f]_{\eta}(\mathbf{y}, t):=f(\mathbf{y}, \eta / 2, t)-f(\mathbf{y},-\eta / 2, t), \quad\langle f\rangle_{\eta}(\mathbf{y}, t):=\frac{1}{2}[f(\mathbf{y}, \eta / 2, t)+f(\mathbf{y},-\eta / 2, t)] .
\end{aligned}
$$

The transmission condition (2.6) can then be written, from the perspective of the region $\Omega_{\eta}^{\mathrm{E}}$ exterior to the layer, in the form

$$
\left\{\mathbf{t}\left(\mathbf{u}_{\eta}\right)\right\}_{\eta}=\mathbf{T}_{\eta}\left\{\mathbf{u}_{\eta}\right\}_{\eta},
$$


where $\mathbf{T}_{\eta}$ is a (nonlocal) DtN transmission operator that can be defined implicitly from the solution of the interior Dirichlet problem in the layer $\Omega_{\eta}^{\mathrm{I}}$.

The next idea is that, as $\eta \rightarrow 0, \mathbf{T}_{\eta}$ becomes local and explicit analytical approximations of $\mathbf{T}_{\eta}$ can be formulated. In other words, we seek local operators $\mathbf{T}_{\eta}^{k}(k \in \mathbb{N})$ that approximate the exact non-local transmission operator $\mathbf{T}_{\eta}$ in the sense that the elastodynamic displacement field $\widetilde{\mathbf{u}}_{\eta}$ in $\Omega_{\eta}^{\mathrm{E}}$ satisfying (2.3), (2.4b) and

$$
\left\{\mathbf{t}\left(\widetilde{\mathbf{u}}_{\eta}\right)\right\}_{\eta}=\mathbf{T}_{\eta}^{k}\left\{\widetilde{\mathbf{u}}_{\eta}\right\}_{\eta} \quad \text { on } \partial \Omega_{\eta}^{\mathrm{E}} \times \mathbb{R}^{+}
$$

approximates the solution $\mathbf{u}_{\eta}^{\mathrm{E}}$ of the exact transmission problem (2.2)-(2.4) and (2.6) within a $O\left(\eta^{k+1}\right)$ error.

As it will be seen later, the operators $\mathbf{T}_{\eta}^{k}$ will involve tangential differential operators on the layer boundary, whose derivation will result from analytically solving differential equations in the rescaled normal coordinate $s:=\nu / \eta$. Tangential and normal derivatives thus need to be distinguished. We accordingly recall definitions of usual tangential differential operators on $\Gamma$ for the three-dimensional case. The tangential gradient $\nabla_{\Gamma}$ and tangential divergence $\operatorname{div}_{\Gamma}$ on $\Gamma$ are defined by

$$
\nabla u(\mathbf{x})=\nabla_{\Gamma} u(\mathbf{x})+(\nabla u(\mathbf{x}) \cdot \mathbf{n}) \mathbf{n}, \quad \operatorname{div} \mathbf{v}(\mathbf{x})=\operatorname{div}_{\Gamma} \mathbf{v}(\mathbf{x})+\nabla(\mathbf{v} \cdot \mathbf{n})(\mathbf{x}) \cdot \mathbf{n}, \quad \mathbf{x}=(\mathbf{y}, 0) \in \Gamma
$$

for any sufficiently regular fields $u$ and $\mathbf{v}$ defined in a neighborhood of $\Gamma$ ( $u$ is scalar, vector- or tensor-valued; $\mathbf{v}$ is vector- or tensor-valued). Letting $\left(\mathbf{e}_{1}, \mathbf{e}_{2}\right)$ denote an orthonormal basis on $\Gamma$ and $\mathbf{y}=y_{1} \mathbf{e}_{1}+y_{2} \mathbf{e}_{2}$, one has

$$
\nabla_{\Gamma} u(\mathbf{x})=\partial_{1} u(\mathbf{x}) \mathbf{e}_{1}+\partial_{2} u(\mathbf{x}) \mathbf{e}_{2}, \quad \operatorname{div}_{\Gamma} \mathbf{v}(\mathbf{x})=\partial_{1} \mathbf{v}(\mathbf{x}) \cdot \mathbf{e}_{1}+\partial_{2} \mathbf{v}(\mathbf{x}) \cdot \mathbf{e}_{2}, \quad \mathbf{x}=(\mathbf{y}, 0) \in \Gamma
$$

Remark 2.2. The $2 \mathrm{D}$ plane strain case (in the $\left(x_{1}, x_{3}\right)$ plane) can be recovered from the $3 \mathrm{D}$ case by (i) setting $\mathbf{u}_{\eta}^{\mathrm{I}} \cdot \mathbf{e}_{2}=\mathbf{u}_{\eta}^{\mathrm{E}} \cdot \mathbf{e}_{2}=0$ and (ii) reducing the spatial dependence of all field variables to coordinates $\left(x_{1}, x_{3}\right)$. Hence, all partial derivatives $\partial_{2}$ are suppressed from the tangential differential operators, whose definition becomes $\nabla_{\Gamma} u=\left(\partial_{1} u\right) \mathbf{e}_{1}$ and $\operatorname{div}_{\Gamma} \mathbf{u}=\left(\partial_{1} \mathbf{u}\right) \cdot \mathbf{e}_{1}$.

A formal derivation (Sect. 3) shows that the generalized jumps of the traction $\mathbf{t}\left(\mathbf{u}_{\eta}^{\mathrm{E}}\right)$ and displacement $\mathbf{u}_{\eta}^{\mathrm{E}}$ on the layer boundary are linked, for any integer $k \geq 1$, by relationships of the form

$$
\begin{aligned}
\eta\left\langle\mathbf{t}^{\mathrm{I}}\left(\mathbf{u}_{\eta}^{\mathrm{I}}\right)\right\rangle_{\eta} & =\sum_{0 \leq 2 j \leq k-1}\left\{\eta^{2 j+1} \mathcal{C}_{2 j+1}\left\langle\mathbf{u}_{\eta}^{\mathrm{E}}\right\rangle_{\eta}+\eta^{2 j} \mathcal{D}_{2 j}\left[\mathbf{u}_{\eta}^{\mathrm{E}}\right]_{\eta}\right\}+O\left(\eta^{k+1}\right) \\
\eta\left[\mathbf{t}^{\mathrm{I}}\left(\mathbf{u}_{\eta}^{\mathrm{I}}\right)\right]_{\eta} & =2 \sum_{1 \leq 2 j+1 \leq k-1}\left\{\eta^{2 j+2} \mathcal{C}_{2 j+2}\left\langle\mathbf{u}_{\eta}^{\mathrm{E}}\right\rangle_{\eta}+\eta^{2 j+1} \mathcal{D}_{2 j+1}\left[\mathbf{u}_{\eta}^{\mathrm{E}}\right]_{\eta}\right\}+O\left(\eta^{k+1}\right) .
\end{aligned}
$$

where $\mathcal{C}_{m} \equiv \mathcal{C}_{m}\left(\nabla_{\Gamma}, \partial_{t}\right)$ and $\mathcal{D}_{m} \equiv \mathcal{D}_{m}\left(\nabla_{\Gamma}, \partial_{t}\right)$ are differential operators of order $m$ whose precise (recursive) definition is given in Lemma 3.5. Neglecting the $O\left(\eta^{k+1}\right)$ remainder in the above formulas yields the definition of the local transmission operator $\mathbf{T}_{\eta}^{k}$. In particular, the case $k=2$ yields the following second-order ETC, on which the remainder of this article will focus:

$$
\left\{\begin{array}{l}
\eta\left\langle\mathbf{t}\left(\widetilde{\mathbf{u}}_{\eta}\right)\right\rangle_{\eta}=A\left[\widetilde{\mathbf{u}}_{\eta}\right]_{\eta}+\eta \mathcal{B}\left(\nabla_{\Gamma}\right)\left\langle\widetilde{\mathbf{u}}_{\eta}\right\rangle_{\eta} \\
\eta\left[\mathbf{t}\left(\widetilde{\mathbf{u}}_{\eta}\right)\right]_{\eta}=\eta \mathcal{B}^{\star}\left(\nabla_{\Gamma}\right)\left[\widetilde{\mathbf{u}}_{\eta}\right]_{\eta}+\eta^{2}\left(\rho^{\mathrm{I}} \partial_{t}^{2}-\mathcal{P}_{2}\left(\nabla_{\Gamma}\right)\right)\left\langle\widetilde{\mathbf{u}}_{\eta}\right\rangle_{\eta}
\end{array} \quad \text { (b) } \quad \text { in } \Gamma \times \mathbb{R}^{+}\right.
$$

where $A \in \mathbb{R}^{3,3}$ is the diagonal matrix

$$
A=\operatorname{Diag}\left(\mu^{\mathrm{I}}, \mu^{\mathrm{I}}, \lambda^{\mathrm{I}}+2 \mu^{\mathrm{I}}\right),
$$

$\mathcal{B}$ and $\mathcal{B}^{\star}$ are first order tangential differential operators, mutually adjoint in $\left(\mathcal{D}(\Gamma)^{3}, \mathcal{D}^{\prime}(\Gamma)^{3}\right)$, given for the 3 -D case by

$$
\begin{aligned}
\mathcal{B}\left(\nabla_{\Gamma}\right) \mathbf{u} & =\mu^{\mathrm{I}} \nabla_{\Gamma}(\mathbf{u} \cdot \mathbf{n})+\lambda^{\mathrm{I}}\left(\operatorname{div}_{\Gamma} \mathbf{u}\right) \mathbf{n}, \\
\mathcal{B}^{\star}\left(\nabla_{\Gamma}\right) \mathbf{u} & =-\lambda^{I} \nabla_{\Gamma}(\mathbf{u} \cdot \mathbf{n})-\mu^{I}\left(\operatorname{div}_{\Gamma} \mathbf{u}\right) \mathbf{n},
\end{aligned}
$$


and $\mathcal{P}_{2}$ is the second order symmetric tangential differential operator given for the 3 -D case by

$$
\mathcal{P}_{2}\left(\nabla_{\Gamma}\right) \mathbf{u}=\left(\lambda^{\mathrm{I}}+\mu^{\mathrm{I}}\right) \nabla_{\Gamma}\left(\operatorname{div}_{\Gamma} \mathbf{u}\right)+\mu^{\mathrm{I}} \Delta_{\Gamma} \mathbf{u}
$$

(in the sequel, the argument $\nabla_{\Gamma}$ of tangential differential operators will often be omitted for brevity, e.g. writing $\mathcal{B}^{\star} \mathbf{u}$ instead of $\left.\mathcal{B}^{\star}\left(\nabla_{\Gamma}\right) \mathbf{u}\right)$. The displacement $\widetilde{\mathbf{u}}_{\eta}$ corresponding to the second-order ETC therefore solves the effective transmission problem (2.3)-(2.4a) and (2.10). The following results provide the theoretical justification of this ETC model by establishing its stability in time and the approximation order achieved by $\widetilde{\mathbf{u}}_{\eta}$.

\subsection{Stability and accuracy of the second-order effective transmission solution}

The rigorous asymptotic analysis of the family of problems (2.2)-(2.4) and (2.6) clearly requires appropriate regularity assumptions on the initial data $\left(\mathbf{u}^{0}, \mathbf{u}^{1}\right)$ and on the coefficients $(\rho, \lambda, \mu)$. Our purpose not being to find the minimal regularity assumptions, we shall restrict ourselves to sufficient conditions. Their formulation requires some concepts and formalism from the regularity theory of linear hyperbolic PDEs ([11] Sect. 7.2, [17]).

\subsubsection{Preliminary notation and recapitulation}

In what follows, we define for each $\eta>0$, the functions $\left(\rho_{\eta}, \lambda_{\eta}, \mu_{\eta}\right)$ as

$$
\left(\rho_{\eta}, \lambda_{\eta}, \mu_{\eta}\right) \equiv(\rho, \lambda, \mu) \text { in } \Omega_{\eta}^{\mathrm{E}}, \quad\left(\rho_{\eta}, \lambda_{\eta}, \mu_{\eta}\right) \equiv\left(\rho^{\mathrm{I}}, \lambda^{\mathrm{I}}, \mu^{\mathrm{I}}\right) \text { in } \Omega_{\eta}^{\mathrm{I}} .
$$

Accordingly, we define, for any $\mathbf{v} \in H^{1}\left(\mathbb{R}^{d}\right)^{d}$

$$
\sigma_{\eta}(\mathbf{v})=\lambda_{\eta} \operatorname{Tr} \varepsilon(\mathbf{v}) \mathrm{I}_{d}+2 \mu_{\eta} \varepsilon(\mathbf{v}) .
$$

Let $D_{\eta}$ be the space (a dense subspace of $L^{2}\left(\mathbb{R}^{d}\right)^{d}$ ) defined by $D_{\eta}=\left\{\mathbf{v} \in H^{1}\left(\mathbb{R}^{d}\right)^{d} / \operatorname{div} \boldsymbol{\sigma}_{\eta}(\mathbf{v}) \in L^{2}\left(\mathbb{R}^{d}\right)^{d}\right\}$, and $\mathcal{A}_{\eta} \in \mathcal{L}\left(D_{\eta}, L^{2}\left(\mathbb{R}^{d}\right)^{d}\right)$ be the differential operator associated to the elasticity system for $\left(\rho_{\eta}, \lambda_{\eta}, \mu_{\eta}\right)$ :

$$
\mathcal{A}_{\eta} \mathbf{v}=\rho_{\eta}^{-1} \operatorname{div} \boldsymbol{\sigma}_{\eta}(\mathbf{v}), \quad \forall \mathbf{v} \in D_{\eta}
$$

Let $D\left(\mathcal{A}_{\eta}\right)=D_{\eta}$. By induction on $k \geq 1$, we then define

$$
\begin{aligned}
D\left(\mathcal{A}_{\eta}^{k+1}\right) & =\left\{\mathbf{v} \in D\left(\mathcal{A}_{\eta}^{k}\right) / \mathcal{A}_{\eta} \mathbf{v} \in D\left(\mathcal{A}_{\eta}^{k}\right)\right\}, \\
D\left(\mathcal{A}_{\eta}^{k+1 / 2}\right) & =\left\{\mathbf{v} \in D\left(\mathcal{A}_{\eta}^{k}\right) / \mathcal{A}_{\eta}^{k} \mathbf{v} \in H^{1}\left(\mathbb{R}^{d}\right)^{d}\right\} .
\end{aligned}
$$

Finally we define the space $D\left(\mathcal{A}_{\eta}^{\infty}\right)$ (also a dense subspace of $\left.L^{2}\left(\mathbb{R}^{d}\right)^{d}\right)$ by

$$
D\left(\mathcal{A}_{\eta}^{\infty}\right)=\bigcap_{k=1}^{\infty} D\left(\mathcal{A}_{\eta}^{k}\right)
$$

In the same way, we define $\mathcal{A}, D(\mathcal{A}), D\left(\mathcal{A}^{k}\right)$ and $D\left(\mathcal{A}^{\infty}\right)$ by simply replacing $\left(\rho_{\eta}, \lambda_{\eta}, \mu_{\eta}\right)$ by $(\rho, \lambda, \mu)$.

From the classical theory of strong solutions of linear second order hyperbolic equations, we know that if the initial data satisfy

$$
\left(\mathbf{u}^{0}, \mathbf{u}^{1}\right) \in D\left(\mathcal{A}_{\eta}^{k}\right) \times D\left(\mathcal{A}_{\eta}^{k-1 / 2}\right), \quad k \geq 1, \quad 2 k \in \mathbb{N}
$$

then the solution $\mathbf{u}_{\eta}$ of $(2.2)-(2.4)$ and (2.6) satisfies

$$
\mathbf{u}_{\eta} \in \bigcap_{m=1}^{2 k} C^{2 k-m}\left(\mathbb{R}^{+} ; D\left(\mathcal{A}_{\eta}^{m / 2}\right)\right) .
$$

Note that a way to ensure (2.19) for any $2 k \in \mathbb{N}$ and any $\eta$ small enough $\left(\eta \leq \eta_{0}\right)$ is to impose that

$$
\left(\mathbf{u}^{0}, \mathbf{u}^{1}\right) \in \bigcap_{k=1}^{+\infty} D\left(\mathcal{A}^{k}\right) \times D\left(\mathcal{A}^{k-1 / 2}\right) \text {, and } \eta_{0}:=\operatorname{dist}\left(\operatorname{supp} \mathbf{u}^{0} \cup \operatorname{supp} \mathbf{u}^{1}, \Gamma\right)>0 .
$$


Next, we denote for any $0 \leq \eta<\eta^{\star}$,

$$
\Omega_{\eta, \eta^{\star}}=\left\{(\mathbf{y}, \nu) \in \mathbb{R}^{d}|\eta / 2<| \nu \mid<\eta^{\star} / 2\right\} .
$$

By Korn's inequality and interior elliptic regularity (e.g. [19], Thms. 10.2 and 4.16), we know that:

$$
(\rho, \lambda, \mu) \in W^{k-1, \infty}\left(\Omega_{0, \eta^{\star}}\right) \quad \Longrightarrow \quad D\left(\mathcal{A}_{\eta}^{k}\right) \subset H^{2 k}\left(\Omega_{\eta, \eta^{\star}} \cup \Omega_{\eta}^{\mathrm{I}}\right)^{d}, \forall \eta<\eta^{\star} .
$$

As a consequence, we deduce that if the coefficients verify

$$
(\rho, \lambda, \mu) \in \bigcap_{k=0}^{+\infty} W^{k, \infty}\left(\Omega_{0, \eta^{\star}}\right)
$$

(implying their infinite smoothness from both sides of $\Gamma$ by virtue of Sobolev embedding e.g. [13], Thm. 7.10) and if (2.21) is satisfied, the field $\mathbf{u}_{\eta}$ has the (local) $C^{\infty}$ regularity property

$$
\mathbf{u}_{\eta} \in C^{m}\left(\mathbb{R}^{+} ; H^{r}\left(\Omega_{\eta, \eta^{\star}} \cup \Omega_{\eta}^{\mathrm{I}}\right)\right), \quad \forall m>0, r>0, \quad \forall \eta<\min \left(\eta_{0}, \eta^{\star}\right) .
$$

Moreover, from energy estimates ([11], Sect. 7.2, Thm. 5), for any $m \geq 1$, one has uniform estimates of the type

$$
\sup _{0 \leq t \leq T}\left\{\left\|\partial_{t}^{m} \mathbf{u}_{\eta}(\cdot, t)\right\|_{H^{r}\left(\Omega_{\eta, \eta^{\star}}\right)}+\left\|\partial_{t}^{m} \mathbf{u}_{\eta}(\cdot, t)\right\|_{H^{r}\left(\Omega_{\eta}^{\mathrm{I}}\right)}\right\} \leq C_{r, m} .
$$

\subsubsection{Main results}

The well-posedness and uniform (in $\eta$ ) stability in time of problems (2.3)-(2.4a) and (2.10) is a preliminary step for the error analysis of the ETCs. It is a consequence (using standard theory of linear evolution equations) of an energy conservation result. In what follows $\mathbf{u} \cdot \mathbf{v}$ denotes the usual scalar product in $\mathbb{R}^{3}$ while $\sigma: \varepsilon$ denotes the scalar product of two $3 \times 3$ matrices: $\boldsymbol{\sigma}: \boldsymbol{\varepsilon}:=\sum_{i, j=1}^{3} \boldsymbol{\sigma}_{i j} \varepsilon_{i j}$. Moreover, it is useful to introduce a somewhat unusual notation: to any vector field $\mathbf{u}$ defined in $\Omega_{\eta}^{\mathrm{E}}$, we associate its "interface discrete gradient" and "interface discrete strain tensor" as the tensor fields on $\Gamma$ defined by:

$$
\nabla_{\eta, \Gamma} \mathbf{u}:=\left(\begin{array}{ccc}
\partial_{1}\left\langle\mathbf{u}_{1}\right\rangle_{\eta} & \partial_{1}\left\langle\mathbf{u}_{2}\right\rangle_{\eta} & \partial_{1}\left\langle\mathbf{u}_{3}\right\rangle_{\eta} \\
\partial_{2}\left\langle\mathbf{u}_{1}\right\rangle_{\eta} & \partial_{2}\left\langle\mathbf{u}_{2}\right\rangle_{\eta} & \partial_{2}\left\langle\mathbf{u}_{3}\right\rangle_{\eta} \\
\eta^{-1}\left[\mathbf{u}_{1}\right]_{\eta} & \eta^{-1}\left[\mathbf{u}_{2}\right]_{\eta} & \eta^{-1}\left[\mathbf{u}_{3}\right]_{\eta}
\end{array}\right), \quad \varepsilon_{\eta, \Gamma}(\mathbf{u}):=\frac{1}{2}\left(\nabla_{\eta, \Gamma} \mathbf{u}+\left(\nabla_{\eta, \Gamma} \mathbf{u}\right)^{T}\right)
$$

In the same way, we shall denote $\nabla_{\Gamma}\langle\mathbf{u}\rangle$ the $2 \times 3$ matrix made of the first two lines of $\nabla_{\eta, \Gamma} \mathbf{u}$.

We can now state (and then prove) our energy conservation result:

Theorem 2.3. Sufficiently regular solutions $\widetilde{\mathbf{u}}_{\eta}$ of (2.3) and (2.4b), and (2.10) satisfy the a priori energy estimate:

$$
\frac{\mathrm{d}}{\mathrm{d} t}\left(\mathcal{E}_{\eta}^{\mathrm{E}}\left(\widetilde{\mathbf{u}}_{\eta}, t\right)+\eta \mathcal{E}_{\eta}^{\mathrm{I}}\left(\widetilde{\mathbf{u}}_{\eta}, t\right)\right)=0
$$

where $\mathcal{E}_{\eta}^{\mathrm{E}}$ is the elastodynamic energy of $\widetilde{\mathbf{u}}_{\eta}$ in the exterior domain $\Omega_{\eta}^{\mathrm{E}}$ :

$$
\mathcal{E}_{\eta}^{\mathrm{E}}\left(\widetilde{\mathbf{u}}_{\eta}, t\right):=\frac{1}{2} \int_{\Omega_{\eta}^{\mathrm{E}}} \rho\left|\partial_{t} \widetilde{\mathbf{u}}_{\eta}\right|^{2} \mathrm{~d} \mathbf{x}+\frac{1}{2} \int_{\Omega_{\eta}^{\mathrm{E}}} \boldsymbol{\sigma}\left(\widetilde{\mathbf{u}}_{\eta}\right): \varepsilon\left(\widetilde{\mathbf{u}}_{\eta}\right) \mathrm{d} \mathbf{x}
$$

and with $\mathcal{E}_{\eta}^{\mathrm{I}}$ defined by $\left(\operatorname{tr} \boldsymbol{\sigma}:=\sum_{i=1}^{3} \boldsymbol{\sigma}_{i i}\right.$ denotes the trace of any $3 \times 3$ matrix $\left.\boldsymbol{\sigma}\right)$

$$
\mathcal{E}_{\eta}^{\mathrm{I}}\left(\widetilde{\mathbf{u}}_{\eta}, t\right):=\frac{1}{2} \int_{\Gamma} \rho^{\mathrm{I}}\left|\left\langle\partial_{t} \widetilde{\mathbf{u}}_{\eta}\right\rangle_{\eta}\right|^{2} \mathrm{~d} \mathbf{y}+\frac{1}{2} \int_{\Gamma}\left(\lambda^{\mathrm{I}}\left(\operatorname{tr} \varepsilon_{\eta, \Gamma}\left(\widetilde{\mathbf{u}}_{\eta}\right)\right)^{2}+2 \mu^{\mathrm{I}} \varepsilon_{\eta, \Gamma}\left(\widetilde{\mathbf{u}}_{\eta}\right): \varepsilon_{\eta, \Gamma}\left(\widetilde{\mathbf{u}}_{\eta}\right)\right) \mathrm{d} \mathbf{y} .
$$

In (2.29) and (2.30), the integrands are evaluated at time $t$.

The proof of this result relies on the following lemma. 
Lemma 2.4. Let $\mathbf{w}$ denote any sufficiently smooth solution of $(2.3)$ in $\Omega_{\eta}^{\mathrm{E}}$. Its energy $\mathcal{E}_{\eta}^{\mathrm{E}}$, defined by $(2.29)$, is such that

$$
\frac{1}{2} \frac{\mathrm{d}}{\mathrm{d} t} \mathcal{E}_{\eta}^{\mathrm{E}}(\mathbf{w}, t)+\int_{\Gamma}\left(\langle\mathbf{t}(\mathbf{w})\rangle_{\eta} \cdot\left[\partial_{t} \mathbf{w}\right]_{\eta}+[\mathbf{t}(\mathbf{w})]_{\eta} \cdot\left\langle\partial_{t} \mathbf{w}\right\rangle_{\eta}\right) \mathrm{d} \mathbf{y}=0 .
$$

Proof. Taking the inner product of the balance equation $\rho \partial_{t}^{2} \mathbf{w}-\operatorname{div} \boldsymbol{\sigma}(\mathbf{w})=0$ with $\partial_{t} \mathbf{w}$, integrating the result over $\Omega_{\eta}^{\mathrm{E}}$ and applying Green's theorem yields (with the unit normal orientation as in Fig. 1)

$$
\rho \int_{\Omega_{\eta}^{\mathrm{E}}} \partial_{t}^{2} \mathbf{w} \cdot \partial_{t} \mathbf{w} \mathrm{d} \mathbf{x}+\int_{\Omega_{\eta}^{\mathrm{E}}} \boldsymbol{\sigma}(\mathbf{w}): \varepsilon\left(\partial_{t} \mathbf{w}\right) \mathrm{d} \mathbf{x}+\int_{\partial \Omega_{\eta}^{+}} \mathbf{t}(\mathbf{w}) \cdot \partial_{t} \mathbf{w} \mathrm{d} \mathbf{y}-\int_{\partial \Omega_{\eta}^{-}} \mathbf{t}(\mathbf{w}) \cdot \partial_{t} \mathbf{w} \mathrm{d} \mathbf{y}=0
$$

Then, identity (2.31) follows from using

$$
\left.\left(\mathbf{t}(\mathbf{w}) \cdot \partial_{t} \mathbf{w}\right)\right|_{\partial \Omega_{\eta}^{+}}-\left.\left(\mathbf{t}(\mathbf{w}) \cdot \partial_{t} \mathbf{w}\right)\right|_{\partial \Omega_{\eta}^{-}}=\langle\mathbf{t}(\mathbf{w})\rangle_{\eta} \cdot\left[\partial_{t} \mathbf{w}\right]_{\eta}+[\mathbf{t}(\mathbf{w})]_{\eta} \cdot\left\langle\partial_{t} \mathbf{w}\right\rangle_{\eta} .
$$

We now prove Theorem 2.3, with the help of Lemma 2.4.

Proof of Theorem 2.3. We start by setting $\mathbf{w}=\widetilde{\mathbf{u}}_{\eta}$ in identity (2.31) of Lemma 2.4 and using the ETCs (2.10) in the integral, to obtain

$$
\begin{aligned}
\frac{1}{2} \frac{\mathrm{d}}{\mathrm{d} t} \mathcal{E}_{\eta}^{\mathrm{E}}\left(\widetilde{\mathbf{u}}_{\eta}, t\right)+\eta \int_{\Gamma}\left(\left(\rho^{\mathrm{I}} \partial_{t}^{2}-\mathcal{P}_{2}\right)\left\langle\widetilde{\mathbf{u}}_{\eta}\right\rangle_{\eta}+\eta^{-1} \mathcal{B}^{\star}\left[\widetilde{\mathbf{u}}_{\eta}\right]_{\eta}\right) \cdot\left\langle\partial_{t} \widetilde{\mathbf{u}}_{\eta}\right\rangle_{\eta} \mathrm{d} \mathbf{y} & \\
& +\int_{\Gamma}\left(\eta^{-1} A\left[\widetilde{\mathbf{u}}_{\eta}\right]_{\eta}+\mathcal{B}\left\langle\widetilde{\mathbf{u}}_{\eta}\right\rangle_{\eta}\right) \cdot\left[\partial_{t} \widetilde{\mathbf{u}}_{\eta}\right]_{\eta} \mathrm{d} \mathbf{y}=0 .
\end{aligned}
$$

Observing, using (2.13) and an integration by parts along $\Gamma$, that

$$
-\int_{\Gamma} \mathcal{P}_{2}\left\langle\widetilde{\mathbf{u}}_{\eta}\right\rangle_{\eta} \cdot\left\langle\partial_{t} \widetilde{\mathbf{u}}_{\eta}\right\rangle_{\eta} \mathrm{d} \mathbf{y}=\frac{1}{2} \frac{\mathrm{d}}{\mathrm{d} t} \int_{\Gamma}\left(\left(\lambda^{\mathrm{I}}+\mu^{\mathrm{I}}\right)\left|\operatorname{div}_{\Gamma}\left\langle\widetilde{\mathbf{u}}_{\eta}\right\rangle_{\eta}\right|^{2}+\mu^{\mathrm{I}}\left|\nabla_{\Gamma}\left\langle\widetilde{\mathbf{u}}_{\eta}\right\rangle_{\eta}\right|^{2}\right) \mathrm{d} \mathbf{y}
$$

and that, since $\mathcal{B}^{\star}$ is the $L^{2}(\Gamma)$-adjoint of $\mathcal{B}$,

$$
\int_{\Gamma}\left(\mathcal{B}^{\star}\left[\widetilde{\mathbf{u}}_{\eta}\right]_{\eta} \cdot\left\langle\partial_{t} \widetilde{\mathbf{u}}_{\eta}\right\rangle_{\eta}+\mathcal{B}\left\langle\widetilde{\mathbf{u}}_{\eta}\right\rangle_{\eta} \cdot\left[\partial_{t} \widetilde{\mathbf{u}}_{\eta}\right]_{\eta}\right) \mathrm{d} \mathbf{y}=\frac{\mathrm{d}}{\mathrm{d} t} \int_{\Gamma} \mathcal{B}\left\langle\widetilde{\mathbf{u}}_{\eta}\right\rangle_{\eta} \cdot\left[\widetilde{\mathbf{u}}_{\eta}\right]_{\eta} \mathrm{d} \mathbf{y}
$$

we easily obtain the identity $(2.28)$ with

$$
\begin{aligned}
\mathcal{E}_{\eta}^{\mathrm{I}}\left(\widetilde{\mathbf{u}}_{\eta}, t\right)= & \frac{1}{2} \int_{\Gamma} \rho^{\mathrm{I}}\left|\left\langle\partial_{t} \widetilde{\mathbf{u}}_{\eta}\right\rangle_{\eta}\right|^{2} \mathrm{~d} \mathbf{y}+\frac{1}{2} \int_{\Gamma}\left(\left(\lambda^{\mathrm{I}}+\mu^{\mathrm{I}}\right)\left|\operatorname{div}_{\Gamma}\left\langle\widetilde{\mathbf{u}}_{\eta}\right\rangle_{\eta}\right|^{2}+\mu^{\mathrm{I}}\left|\nabla_{\Gamma}\left\langle\widetilde{\mathbf{u}}_{\eta}\right\rangle_{\eta}\right|^{2}\right) \mathrm{d} \mathbf{y} \\
& +\int_{\Gamma}\left(\frac{1}{2} \eta^{-2} A\left[\widetilde{\mathbf{u}}_{\eta}\right]_{\eta} \cdot\left[\widetilde{\mathbf{u}}_{\eta}\right]_{\eta}+\eta^{-1} \mathcal{B}\left\langle\widetilde{\mathbf{u}}_{\eta}\right\rangle_{\eta} \cdot\left[\widetilde{\mathbf{u}}_{\eta}\right]_{\eta}\right) \mathrm{d} \mathbf{y} .
\end{aligned}
$$

We conclude by making the nice observation that, using expressions (2.11) of the matrix $A$ and (2.12) of the operator $\mathcal{B}, \mathcal{E}_{\eta}^{\mathrm{I}}\left(\widetilde{\mathbf{u}}_{\eta}, t\right)$ can be recast in the form (2.30); the details of this derivation are left to the reader.

In Section 4, we prove the following error estimates for $\mathbf{u}_{\eta}^{\mathrm{E}}-\widetilde{\mathbf{u}}_{\eta}$ in the energy norm, where $\mathbf{u}_{\eta}^{\mathrm{E}}$ is the solution of the exact problem (2.2)-(2.4) and (2.6) whereas $\widetilde{\mathbf{u}}_{\eta}$ is the solution of the approximate model with conditions (2.10). For this, we are led to make some regularity assumptions on the initial data $\left(\mathbf{u}_{0}, \mathbf{u}_{1}\right)$.

Theorem 2.5. Assume that the initial data $\left(\mathbf{u}^{0}, \mathbf{u}^{1}\right)$ satisfy the regularity assumption (2.21) and the coefficients in the reference medium satisfy the regularity assumption (2.24). Let $\widetilde{\mathbf{u}}_{\eta}$ be the solution of the approximate problem (2.3)-(2.4a) and (2.10) and let $\mathbf{u}_{\eta}^{\mathrm{E}}$ the solution of the exact problem (2.2)-(2.4) and (2.6). There exists, for any duration $T \in\left[0,+\infty\left[\right.\right.$, a constant $C(T)>0$ depending on $\left(\mathbf{u}^{0}, \mathbf{u}^{1}\right),(\rho, \lambda, \mu)$ and $\left(\rho^{\mathrm{I}}, \lambda^{\mathrm{I}}, \mu^{\mathrm{I}}\right)$ and independent of $\eta$, such that:

$$
\sup _{0 \leqslant t \leqslant T}\left\{\left\|\partial_{t}\left(\mathbf{u}_{\eta}^{\mathrm{E}}-\widetilde{\mathbf{u}}_{\eta}\right)(\cdot, t)\right\|_{L^{2}\left(\Omega_{\eta}^{\mathrm{E}}\right)}+\left\|\left(\mathbf{u}_{\eta}^{\mathrm{E}}-\widetilde{\mathbf{u}}_{\eta}\right)(\cdot, t)\right\|_{H^{1}\left(\Omega_{\eta}^{\mathrm{E}}\right)}\right\} \leqslant C(T) \eta^{3} .
$$


Remark 2.6. It is clear that the very strong regularity assumptions (2.21) and (2.24) are not necessary for Theorem 2.5 to hold. However, they appear more or less necessary to justify at any order the formal asymptotic expansion of Section 3.1. A possible, albeit tedious, study might consist in determining by how much such assumptions can be weakened. Solving this issue is of marginal usefulness at best for the expected applications of this work.

\section{Derivation of the EFFECTIVE transmission CONDitions}

In this section, a formal asymptotic expansion for the displacement $\mathbf{u}_{\eta}^{\mathrm{I}}$ is derived and used for defining equivalent transmission conditions. These results will then be justified rigorously in Section 4 .

\subsection{Scaled asymptotic expansion}

We start by considering an asymptotic expansion in powers of the layer thickness $\eta$ for the interior solution $\mathbf{u}_{\eta}^{\mathrm{I}}$, making the following ansatz:

$$
\mathbf{u}_{\eta}^{\mathrm{I}}(\mathbf{x}, t)=\mathbf{U}^{0}\left(\mathbf{y}, \eta^{-1} \nu, t\right)+\eta \mathbf{U}^{1}\left(\mathbf{y}, \eta^{-1} \nu, t\right)+\eta^{2} \mathbf{U}^{2}\left(\mathbf{y}, \eta^{-1} \nu, t\right)+\ldots \quad \mathbf{x}=(\mathbf{y}, \nu) \in \Omega_{\eta}^{\mathrm{I}} .
$$

where the $\mathbf{U}^{k}$ are defined on $\Gamma \times\left[-\frac{1}{2}, \frac{1}{2}\right] \times \mathbb{R}^{+}$. The ansatz (3.1) implies in particular analogous expansions for the traces of the displacement and the traction vectors on the layer boundary. We therefore have

$$
\mathbf{u}_{\eta}^{\mathrm{I}}(\mathbf{y}, \pm \eta / 2, t)=\mathbf{u}_{ \pm}^{0}(\mathbf{y}, t)+\eta \mathbf{u}_{ \pm}^{1}(\mathbf{y}, t)+\eta^{2} \mathbf{u}_{ \pm}^{2}(\mathbf{y}, t)+\ldots
$$

where the $\mathbf{u}_{ \pm}^{\ell}$ are defined on $\partial \Omega_{\eta}^{ \pm} \times \mathbb{R}^{+}$as the traces of the $\mathbf{U}^{\ell}: \mathbf{u}_{ \pm}^{\ell}(\mathbf{y}, t)=\mathbf{U}^{\ell}\left(\mathbf{y}, \pm \frac{1}{2}, t\right)$, and

$$
\mathbf{t}^{\mathrm{I}}\left(\mathbf{u}_{\eta}^{\mathrm{I}}\right)(\mathbf{y}, \pm \eta / 2, t)=\mathbf{t}_{ \pm}^{0}(\mathbf{y}, t)+\eta \mathbf{t}_{ \pm}^{1}(\mathbf{y}, t)+\eta^{2} \mathbf{t}_{ \pm}^{2}(\mathbf{y}, t)+\ldots
$$

Remark 3.1. We consider for this section an expansion of the interior solution $\mathbf{u}_{\eta}^{\mathrm{I}}$ only. An asymptotic expansion of the exterior solution $\mathbf{u}_{\eta}^{\mathrm{E}}$ will be needed later and established in Section 4.

Remark 3.2. By analogy with the generalized jumps and averages defined in (2.7), we shall denote (all quantities depend on $(\mathbf{y}, t)$ but this dependence is omitted for simplicity):

$$
\left\{\mathbf{u}^{k}\right\}=\left(\left\langle\mathbf{u}^{k}\right\rangle,\left[\mathbf{u}^{k}\right]\right), \quad \text { with }\left\langle\mathbf{u}^{k}\right\rangle=\frac{1}{2}\left(\mathbf{u}_{+}^{k}+\mathbf{u}_{-}^{k}\right), \quad\left[\mathbf{u}^{k}\right]=\mathbf{u}_{+}^{k}-\mathbf{u}_{-}^{k} .
$$

and the same for $\left\{\mathbf{t}^{k}\right\}:=\left(\left\langle\mathbf{t}^{k}\right\rangle,\left[\mathbf{t}^{k}\right]\right)$ in such a way that, as a consequence of (3.2) and (3.3)

$$
\left\{\mathbf{u}_{\eta}^{\mathrm{I}}\right\}_{\eta}=\left\{\mathbf{u}^{0}\right\}+\eta\left\{\mathbf{u}^{1}\right\}+\eta^{2}\left\{\mathbf{u}^{2}\right\}+\ldots, \quad\left\{\mathbf{t}^{\mathrm{I}}\left(\mathbf{u}_{\eta}^{\mathrm{I}}\right)\right\}_{\eta}=\left\{\mathbf{t}^{0}\right\}+\eta\left\{\mathbf{t}^{1}\right\}+\eta^{2}\left\{\mathbf{t}^{2}\right\}+\ldots
$$

The scaling $s=\nu / \eta$ is now introduced in the representation (2.5), in order to express the ansatz (3.1) in terms of coordinates $(\mathbf{y}, s)$ in the fixed normalized strip $\Omega_{1}^{\mathrm{I}}$ :

$$
(\mathbf{y}, \nu) \in \Omega_{\eta}^{\mathrm{I}}=\Gamma \times[-\eta / 2, \eta / 2] \mapsto(\mathbf{y}, s) \in \Omega_{1}^{\mathrm{I}}=\Gamma \times\left[-\frac{1}{2}, \frac{1}{2}\right] .
$$

This scaling implies the differentiation rule $\partial_{\nu} \leftrightarrow \eta^{-1} \partial_{s}$. To obtain equations for the $\mathbf{U}^{k}$ involved in the ansatz (3.1), the governing equations inside the layer must be rescaled accordingly. To this purpose, the tangential and normal derivatives in the interior balance equation (2.2) and the constitutive equation (2.4) are first separated using definitions (2.9). In particular, one computes that

$$
\operatorname{div} \boldsymbol{\sigma}\left(\mathbf{u}_{\eta}^{\mathrm{I}}\right)=\mathcal{P}_{2} \mathbf{u}_{\eta}^{\mathrm{I}}+\mathcal{P}_{1} \partial_{\nu} \mathbf{u}_{\eta}^{\mathrm{I}}+A \partial_{\nu}^{2} \mathbf{u}_{\eta}^{\mathrm{I}},
$$


with $A, \mathcal{P}_{2}$ as given by (2.11) and (2.13) and where $\mathcal{P}_{1}$ is the skew-symmetric first-order tangential differential operator

$$
\mathcal{P}_{1}\left(\nabla_{\Gamma}\right) \mathbf{u}=\mathcal{B}\left(\nabla_{\Gamma}\right)-\mathcal{B}^{\star}\left(\nabla_{\Gamma}\right) \equiv\left(\lambda^{\mathrm{I}}+\mu^{\mathrm{I}}\right)\left(\nabla_{\Gamma}(\mathbf{u} \cdot \mathbf{n})+\left(\operatorname{div}_{\Gamma} \mathbf{u}\right) \mathbf{n}\right)
$$

(with $\mathcal{B}$ as defined in (2.12)), while the traction vector on $\partial \Omega_{\eta}^{\mathrm{E}}$ is given by

$$
\mathbf{t}^{\mathrm{I}}\left(\mathbf{u}_{\eta}^{\mathrm{I}}\right)=\mathcal{B} \mathbf{u}_{\eta}^{\mathrm{I}}+A \partial_{\nu} \mathbf{u}_{\eta}^{\mathrm{I}} .
$$

Finally, equation $(2.2)$ is found to take the form

$$
\rho^{\mathrm{I}} \partial_{t}^{2} \mathbf{u}_{\eta}^{\mathrm{I}}-\mathcal{P}_{2} \mathbf{u}_{\eta}^{\mathrm{I}}-\mathcal{P}_{1} \partial_{\nu} \mathbf{u}_{\eta}^{\mathrm{I}}-A \partial_{\nu}^{2} \mathbf{u}_{\eta}^{\mathrm{I}}=0
$$

Now, we substitute the ansatz (3.1) in equations (3.9), (3.2) and (3.3) (using (3.8) and rescaling the normal derivatives according to (3.5)). The equations below (where the label (a) refers to equations inside the layers, (b) to the continuity of displacements and (c) to the continuity of tractions) satisfied by each $\mathbf{U}^{k}$ in the rescaled layer $\left.\Omega_{1}^{\mathrm{I}}:=\mathbb{R}^{d-1} \times\right]-1 / 2,1 / 2\left[\right.$ (i.e. $\Omega_{\eta}^{\mathrm{I}}$ with $\eta=1$ ) and on its boundary $\partial \Omega_{1}^{ \pm}:=\mathbb{R}^{d-1} \times\{ \pm 1 / 2\}$ are then found by a term-by-term identification of the resulting expansions in powers on $\eta$ obtained from (3.9) (for equations (a)), (3.2) (for equations (b)) and (3.3) (for equations (c)):

$$
\begin{aligned}
& \text { (a) } \quad A \partial_{s}^{2} \mathbf{U}^{0}=0 \\
& \text { (b) } \quad\left\{\mathbf{U}^{0}\right\}_{1}=\left\{\mathbf{u}^{0}\right\} \\
& \text { (c) } \quad A \partial_{s} \mathbf{U}^{0}=0 \\
& \text { (a) } \quad A \partial_{s}^{2} \mathbf{U}^{1}=-\mathcal{P}_{1} \partial_{s} \mathbf{U}^{0} \\
& \text { (b) } \quad\left\{\mathbf{U}^{1}\right\}_{1}=\left\{\mathbf{u}^{1}\right\} \\
& \mathbf{t}_{ \pm}^{0}=A \partial_{s} \mathbf{U}^{1}+\mathcal{B} \mathbf{U}^{0} \\
& \text { in } \Omega_{1}^{\mathrm{I}} \\
& \text { on } \partial \Omega_{1}^{ \pm} \quad(k=0) \\
& \text { on } \partial \Omega_{1}^{ \pm} \\
& A \partial_{s}^{2} \mathbf{U}^{k}=\left(\rho^{\mathrm{I}} \partial_{t}^{2}-\mathcal{P}_{2}\right) \mathbf{U}^{k-2}-\mathcal{P}_{1} \partial_{s} \mathbf{U}^{k-1} \\
& \text { in } \Omega_{1}^{\mathrm{I}} \\
& \text { on } \partial \Omega_{1}^{ \pm} \quad(k=1) \\
& \text { on } \partial \Omega_{1}^{ \pm} \\
& \text {(b) } \quad\left\{\mathbf{U}^{k}\right\}_{1}=\left\{\mathbf{u}^{k}\right\} \\
& \text { (c) } \quad \mathbf{t}_{ \pm}^{k-1}=A \partial_{s} \mathbf{U}^{k}+\mathcal{B} \mathbf{U}^{k-1} \\
& \text { in } \Omega_{1}^{\mathrm{I}} \\
& \text { on } \partial \Omega_{1}^{ \pm} \quad(k \geqslant 2) \\
& \text { on } \partial \Omega_{1}^{ \pm}
\end{aligned}
$$

Systems (3.10)), ((3.11)) and (3.12) are exploited analytically, by induction on $k$. This can be done in any of several ways; the one we adopt here is in the spirit of constructing DtN operators. In each system (3.11) and (3.12), the subsystem $(\mathrm{a}, \mathrm{b})$ is then seen as a Dirichlet problem inside the layer for the differential operator $\partial_{s}^{2}$ (the variables $(\mathbf{y}, t)$ being treated as parameters). Solving these systems for the $\mathbf{U}^{k}$ yields them in function of the exterior Dirichlet traces; more precisely, $\mathbf{U}^{k}$ is found as a $k$ th degree polynomial in $s$ whose coefficients depend on the exterior traces $\left\{\mathbf{u}^{\ell}\right\}(0 \leq \ell \leq k)$. Then, equation (c) allows us to compute the traction coefficients $\mathbf{t}_{ \pm}^{k-1}$ (there is an natural index shift due to normal differentiation) as functions of the same $\left\{\mathbf{u}^{\ell}\right\}(0 \leq \ell \leq k)$.

System $(3.10)(k=0)$. The case $k=0$ has a particular status in the sense that it simply allows to check that, since the limit problem when $\eta$ tends to 0 consists in simply ignoring the presence of the layer, the zeroth-order contribution to the displacement field is continuous. Indeed, solving (3.10a) and (3.10b) leads to

$$
\mathbf{U}^{0}(\mathbf{y}, s, t)=\left\langle\mathbf{u}^{0}\right\rangle+\left[\mathbf{u}^{0}\right] s .
$$

(the dependence on $(\mathbf{y}, t)$ of jumps and averages, e.g. $\left[\mathbf{u}^{0}\right]$ or $\left\langle\mathbf{t}^{1}\right\rangle$, being suppressed here and thereafter for notational convenience). Then equation (3.10c) yields

$$
\left[\mathbf{u}^{0}\right]=0, \quad \mathbf{U}^{0}(\mathbf{y}, s, t)=\left\langle\mathbf{u}^{0}\right\rangle, \quad(\mathbf{y}, s) \in \Omega_{1}^{\mathrm{I}}, t \in \mathbb{R}_{+} .
$$

System (3.11) $(k=1)$. Solving equations (3.11a) and (3.11b), with $\mathbf{U}^{0}$ given by (3.13), yields

$$
\forall(\mathbf{y}, s, t) \in \mathbb{R}^{d-1} \times\left[-\frac{1}{2}, \frac{1}{2}\right] \times \mathbb{R}_{+}, \quad \mathbf{U}^{1}(\mathbf{y}, s, t)=\left\langle\mathbf{u}^{1}\right\rangle+\left[\mathbf{u}^{1}\right] s .
$$


Then $\partial_{s} \mathbf{U}^{1}=\left[\mathbf{u}^{1}\right]$, so that, using (3.13), equation (3.11c) provides the relationships

$$
\left[\mathbf{t}^{0}\right]=0, \quad\left\langle\mathbf{t}^{0}\right\rangle=A\left[\mathbf{u}^{1}\right]+\mathcal{B}\left\langle\mathbf{u}^{0}\right\rangle .
$$

System (3.12) ( $k \geq 2$ ). Equations (3.12a) and (3.12b) can be solved recursively for any $k \geq 2$, as summarized in the following lemma whose proof is deferred to Appendix 6. In order to state this lemma in a concise way, it is useful to introduce the two tangential differential operators

$$
\mathcal{Q}_{1} \equiv \mathcal{Q}_{1}\left(\nabla_{\Gamma}\right):=-A^{-1} \mathcal{P}_{1}, \quad \mathcal{Q}_{2} \equiv \mathcal{Q}_{2}\left(\nabla_{\Gamma}, \partial_{t}\right):=A^{-1}\left(\rho^{\mathrm{I}} \partial_{t}^{2}-\mathcal{P}_{2}\right)
$$

Lemma 3.3. The solution to the Dirichlet problem (3.12a) and (3.12b) is given by

$$
\mathbf{U}^{\ell}(\mathbf{y}, s, t)=\sum_{j=0}^{\ell}\left\{\mathcal{X}_{j}\left(s, \nabla_{\Gamma}, \partial_{t}\right)\left\langle\mathbf{u}^{\ell-j}\right\rangle+\mathcal{Y}_{j-1}\left(s, \nabla_{\Gamma}, \partial_{t}\right)\left[\mathbf{u}^{\ell-j+1}\right]\right\}
$$

where the differential operators $\mathcal{X}_{j}(s) \equiv \mathcal{X}_{j}\left(s, \nabla_{\Gamma}, \partial_{t}\right)$ and $\mathcal{Y}_{j-1}(s) \equiv \mathcal{Y}_{j-1}\left(s, \nabla_{\Gamma}, \partial_{t}\right)(j \geq 0)$ are defined recursively by the relations

$$
\begin{gathered}
\mathcal{X}_{0}(s)=1, \quad \mathcal{X}_{1}(s)=0, \quad \mathcal{Y}_{-1}(s)=0, \quad \mathcal{Y}_{0}(s)=s, \\
\mathcal{X}_{j}(s)=S\left(\mathcal{Q}_{1} \partial_{s} \mathcal{X}_{j-1}(s)+\mathcal{Q}_{2} \mathcal{X}_{j-2}(s)\right), \quad \mathcal{Y}_{j-1}(s)=S\left(\mathcal{Q}_{1} \partial_{s} \mathcal{Y}_{j-2}(s)+\mathcal{Q}_{2} \mathcal{Y}_{j-3}(s)\right) \quad(j \geq 2),
\end{gathered}
$$

with the linear operator $S$ defined for any operator-valued function $s \mapsto \mathcal{F}(s)$ by

$$
[S \mathcal{F}](s)=\int_{-1 / 2}^{s}\left(s-\frac{1}{2}\right)\left(s^{\prime}+\frac{1}{2}\right) \mathcal{F}\left(s^{\prime}\right) \mathrm{d} s^{\prime} .+\int_{s}^{1 / 2}\left(s+\frac{1}{2}\right)\left(s^{\prime}-\frac{1}{2}\right) \mathcal{F}\left(s^{\prime}\right) \mathrm{d} s^{\prime} .
$$

More precisely, $\mathcal{X}_{j}(s)$ and $\mathcal{Y}_{j}(s)$ are (operator-valued) polynomials in $s$ of degree $j$ and $j+1$, respectively, having boundary values $\mathcal{X}_{j}\left( \pm \frac{1}{2}\right)=\mathcal{Y}_{j-1}\left( \pm \frac{1}{2}\right)=0(j \geq 2)$ and verifying the parity properties

$$
\mathcal{X}_{j}(-s)=(-1)^{j} \mathcal{X}_{j}(s), \quad \mathcal{Y}_{j}(-s)=(-1)^{j+1} \mathcal{Y}_{j}(s)
$$

Moreover for each $s, \mathcal{X}_{j}(s)$ and $\mathcal{Y}_{j}(s)$ are homogeneous linear differential operators in $(\mathbf{y}, t)$ of order $j$.

Remark 3.4. With the definitions of Lemma 3.3, equation (3.17) for $k=0,1$ agrees with (3.13) and (3.14), respectively. Moreover, it is shown in Appendix A that:

$$
\mathcal{X}_{2}(s)=\frac{1}{2}\left(s^{2}-\frac{1}{4}\right) \mathcal{Q}_{2}, \quad \mathcal{Y}_{1}(s)=\frac{1}{2}\left(s^{2}-\frac{1}{4}\right) \mathcal{Q}_{1} .
$$

\subsection{Derivation of equivalent transmission conditions}

Let us introduce the differential operators $\mathcal{C}_{j} \equiv \mathcal{C}_{j}\left(\nabla_{\Gamma}, \partial_{t}\right)$ and $\mathcal{D}_{j} \equiv \mathcal{D}_{j}\left(\nabla_{\Gamma}, \partial_{t}\right)$ of order $j$ given by

$$
\mathcal{C}_{j}\left(\nabla_{\Gamma}, \partial_{t}\right)=A \partial_{s} \mathcal{X}_{j}\left(\frac{1}{2}, \nabla_{\Gamma}, \partial_{t}\right)+\delta_{j, 1} \mathcal{B}\left(\nabla_{\Gamma}\right) . \quad \mathcal{D}_{j}\left(\nabla_{\Gamma}, \partial_{t}\right)=A \partial_{s} \mathcal{Y}_{j}\left(\frac{1}{2}, \nabla_{\Gamma}, \partial_{t}\right)+\frac{1}{2} \delta_{j, 1} \mathcal{B}\left(\nabla_{\Gamma}\right)
$$

In particular, using (3.18a), (3.19) and (3.7), we compute that:

$$
\mathcal{C}_{1}=\mathcal{B}, \quad \mathcal{C}_{2}=\frac{1}{2}\left(\rho^{\mathrm{I}} \partial_{t}^{2}-\mathcal{P}_{2}\right), \quad \mathcal{D}_{0}=A, \quad \mathcal{D}_{1}=\frac{1}{2} \mathcal{B}^{\star} .
$$


Lemma 3.5. The coefficients $\mathbf{t}_{ \pm}^{\ell}$ in expansion (3.3) are such that their averages and jumps, as defined in Remark 3.2, are given by

$$
\begin{aligned}
\left\langle\mathbf{t}^{\ell}\right\rangle & =\sum_{0 \leq 2 j \leq \ell}\left\{\mathcal{C}_{2 j+1}\left\langle\mathbf{u}^{\ell-2 j}\right\rangle+\mathcal{D}_{2 j}\left[\mathbf{u}^{\ell-2 j+1}\right]\right\}, \\
{\left[\mathbf{t}^{\ell}\right] } & =\sum_{1 \leq 2 j+1 \leq \ell} 2\left\{\mathcal{C}_{2 j+2}\left\langle\mathbf{u}^{\ell-2 j-1}\right\rangle+\mathcal{D}_{2 j+1}\left[\mathbf{u}^{\ell-2 j}\right]\right\} .
\end{aligned}
$$

Proof. The proof requires computing $\mathbf{t}_{ \pm}^{\ell}$ as given by (3.12c), which in turn needs formulas for $\partial_{s} \mathbf{U}^{\ell}\left( \pm \frac{1}{2}, \mathbf{y}, t\right)$. Differentiating (3.17) with respect to $s$, evaluating the result for $s= \pm \frac{1}{2}$ and noting that $\partial_{s} \mathcal{X}_{0}=\partial_{s} \mathcal{Y}_{-1}=0$ by virtue of definitions (3.18a) and (3.20) yields

$$
A \partial_{s} \mathbf{U}^{\ell}\left(\mathbf{y}, \pm \frac{1}{2}, t\right)=A \sum_{j=1}^{\ell}\left\{\partial_{s} \mathcal{X}_{j}\left( \pm \frac{1}{2}\right)\left\langle\mathbf{u}^{\ell-j}\right\rangle+\partial_{s} \mathcal{Y}_{j-1}\left( \pm \frac{1}{2}\right)\left[\mathbf{u}^{\ell-j+1}\right]\right\} .
$$

Then, we rewrite the above sum by (i) exploiting for $s=-\frac{1}{2}$ the parity properties

$$
\partial_{s} \mathcal{X}_{j}\left(-\frac{1}{2}\right)=(-1)^{j+1} \partial_{s} \mathcal{X}_{j}\left(\frac{1}{2}\right), \quad \partial_{s} \mathcal{Y}_{j}\left(-\frac{1}{2}\right)=(-1)^{j} \partial_{s} \mathcal{Y}_{j}\left(\frac{1}{2}\right)
$$

that follow directly from (3.18c), (ii) expressing $\mathcal{X}_{j}\left(\frac{1}{2}\right), \mathcal{Y}_{j}\left(\frac{1}{2}\right)$ in terms of $\mathcal{C}_{j}, \mathcal{D}_{j}$ by invoking (3.20), and (iii) noting that $\left\langle\mathbf{u}^{\ell-1}\right\rangle \pm \frac{1}{2}\left[\mathbf{u}^{\ell-1}\right]=\mathbf{U}^{\ell-1}\left( \pm \frac{1}{2}\right)$, to obtain

$$
A \partial_{s} \mathbf{U}^{\ell}\left(\mathbf{y}, \pm \frac{1}{2}, t\right)=\sum_{j=1}^{\ell}( \pm 1)^{j+1}\left\{\mathcal{C}_{j}\left\langle\mathbf{u}^{\ell-j}\right\rangle+\mathcal{D}_{j-1}\left[\mathbf{u}^{\ell-j+1}\right]\right\}-\mathcal{B} \mathbf{U}^{\ell-1}\left(\mathbf{y}, \pm \frac{1}{2}, t\right)
$$

Now, we evaluate $\mathbf{t}_{ \pm}^{\ell}$ by applying (3.12c) with $k=\ell+1$, with the help of (3.22) with $\ell$ replaced by $\ell+1$, to obtain

$$
\mathbf{t}_{ \pm}^{\ell}=\sum_{j=1}^{\ell+1}( \pm 1)^{j+1}\left\{\mathcal{C}_{j}\left\langle\mathbf{u}^{\ell+1-j}\right\rangle+\mathcal{D}_{j-1}\left[\mathbf{u}^{\ell+2-j}\right]\right\}
$$

The sought results (3.21a) and (3.21b) finally follow straightforwardly from computing $\left\langle\mathbf{t}^{\ell}\right\rangle=\left(\mathbf{t}_{+}^{\ell}+\mathbf{t}_{-}^{\ell}\right) / 2$ and $\left[\mathbf{t}^{\ell}\right]=\mathbf{t}_{+}^{\ell}-\mathbf{t}_{-}^{\ell}$ by means of the above expression of $\mathbf{t}_{ \pm}^{\ell}$.

Now, let expansion (3.3) be evaluated with the help of Lemma 3.5. Multiplying (3.21a) and (3.21b) by $\eta^{\ell}$, summing the resulting identities over $0 \leq \ell \leq k$, using where relevant that $\left[\mathbf{u}_{ \pm}^{0}\right]=0$ and inverting the sums over $j$ and $\ell$, one obtains after some manipulations

$$
\begin{aligned}
\left\langle\mathbf{t}_{\eta}^{(k)}\right\rangle:=\sum_{\ell=0}^{k} \eta^{\ell}\left\langle\mathbf{t}^{\ell}\right\rangle & =\sum_{0 \leq 2 j \leq k} \sum_{\ell=2 j}^{k} \eta^{\ell}\left\{\mathcal{C}_{2 j+1}\left\langle\mathbf{u}^{\ell-2 j}\right\rangle+\mathcal{D}_{2 j}\left[\mathbf{u}^{\ell-2 j+1}\right]\right\} \\
& =\sum_{0 \leq 2 j \leq k}\left\{\eta^{2 j} \mathcal{C}_{2 j+1}\left\langle\mathbf{u}_{\eta}^{(k-2 j)}\right\rangle+\eta^{2 j-1} \mathcal{D}_{2 j}\left[\mathbf{u}_{\eta}^{(k-2 j+1)}\right]\right\} \\
{\left[\mathbf{t}_{\eta}^{(k)}\right]:=\sum_{\ell=0}^{k} \eta^{\ell}\left[\mathbf{t}^{\ell}\right] } & =\sum_{1 \leq 2 j+1 \leq k} \sum_{\ell=2 j+1}^{k} 2 \eta^{\ell}\left\{\mathcal{C}_{2 j+2}\left\langle\mathbf{u}^{\ell-2 j-1}\right\rangle+\mathcal{D}_{2 j+1}\left[\mathbf{u}^{\ell-2 j}\right]\right\} \\
& =\sum_{1 \leq 2 j+1 \leq k} 2\left\{\eta^{2 j+1} \mathcal{C}_{2 j+2}\left\langle\mathbf{u}_{\eta}^{k-2 j-1}\right\rangle+\eta^{2 j} \mathcal{D}_{2 j+1}\left[\mathbf{u}_{\eta}^{k-2 j}\right]\right\},
\end{aligned}
$$


where $\left\langle\mathbf{u}_{\eta}^{(k)}\right\rangle:=\sum_{\ell=0}^{k} \eta^{\ell}\left\langle\mathbf{u}^{\ell}\right\rangle$ and $\left[\mathbf{u}_{\eta}^{(k)}\right]:=\sum_{\ell=0}^{k} \eta^{\ell}\left[\mathbf{u}^{\ell}\right]$. Now, assuming that, according to what is expected,

$$
\begin{array}{r}
\mathbf{u}_{\eta}^{\mathrm{I}}(\mathbf{y}, \pm \eta / 2, t)-\left(\mathbf{u}_{ \pm}^{0}(\mathbf{y}, t)+\eta \mathbf{u}_{ \pm}^{1}(\mathbf{y}, t) \ldots+\eta^{m} \mathbf{u}_{ \pm}^{m}(\mathbf{y}, t)\right)=O\left(\eta^{m+1}\right) \\
\mathbf{t}^{\mathrm{I}}\left(\mathbf{u}_{\eta}^{\mathrm{I}}\right)(\mathbf{y}, \pm \eta / 2, t)-\left(\mathbf{t}_{ \pm}^{0}(\mathbf{y}, t)+\eta \mathbf{t}_{ \pm}^{1}(\mathbf{y}, t) \ldots+\eta^{m} \mathbf{t}_{ \pm}^{m}(\mathbf{y}, t)\right)=O\left(\eta^{m+1}\right)
\end{array}
$$

hold for any integer $m$, equations (3.23a) and (3.23b) can be recast in the form

$$
\begin{aligned}
\eta\left\langle\mathbf{t}^{\mathrm{I}}\left(\mathbf{u}_{\eta}^{\mathrm{I}}\right)\right\rangle_{\eta} & =\sum_{0 \leq 2 j \leq k-1}\left\{\eta^{2 j+1} \mathcal{C}_{2 j+1}\left\langle\mathbf{u}_{\eta}^{\mathrm{I}}\right\rangle+\eta^{2 j} \mathcal{D}_{2 j}\left[\mathbf{u}_{\eta}^{\mathrm{I}}\right]\right\}+O\left(\eta^{k+1}\right) \\
\eta\left[\mathbf{t}^{\mathrm{I}}\left(\mathbf{u}_{\eta}^{\mathrm{I}}\right)\right]_{\eta} & =2 \sum_{1 \leq 2 j+1 \leq k-1}\left\{\eta^{2 j+2} \mathcal{C}_{2 j+2}\left\langle\mathbf{u}_{\eta}^{\mathrm{I}}\right\rangle+\eta^{2 j+1} \mathcal{D}_{2 j+1}\left[\mathbf{u}_{\eta}^{\mathrm{I}}\right]\right\}+O\left(\eta^{k+1}\right) .
\end{aligned}
$$

For given $k$, these identities therefore define, upon dropping the $O\left(\eta^{k+1}\right)$ term, an approximate local transmission operator $\mathbf{T}_{\eta}^{k}$ which is formally of order $k+1$ in accuracy and involves differential operators in $(\mathbf{y}, t)$ of order $k$, while the limiting case $k \rightarrow+\infty$ formally defines the exact nonlocal transmission operator $\mathbf{T}_{\eta}$ involved in (2.8).

In particular, setting $k=2$ in the above relations yields the equivalent transmission conditions (2.10), on which we will focus for the remainder of this article.

We will prove that the system (2.3), (2.4a), and (2.10) is well-posed, uniformly stable (when $\eta$ varies) and that its solution $\widetilde{\mathbf{u}}_{\eta}$ approximates, under suitable assumptions, the exact transmission solution $\mathbf{u}_{\eta}$ within an $O\left(\eta^{3}\right)$ error.

Remark 3.6. Using the approximate operator $\mathbf{T}_{\eta}^{k}$ a priori defines approximate transmission conditions of arbitrary accuracy. However, it is not clear at all that such transmission conditions lead to well-posed and uniformly stable evolution problems when $k>2$ : the example of boundary value problems for the wave equation [24] even suggests that it should not be the case. Transforming the transmission conditions issued from (3.24a) and (3.24b), such as replacing Taylor expansions by Padé-like approximations (as in the case of absorbing boundary conditions [9] or generalized impedance conditions for strongly absorbing obstacles [16]) might remedy this problem. This is however out of the scope of this paper.

\section{Asymptotic Analysis of the ETC of order 2}

The aim of this section is to prove Theorem 2.5, i.e. justify the order of approximation between the solution of the exact transmission solution $\mathbf{u}_{\eta}^{\mathrm{E}}$ and the effective transmission solution $\widetilde{\mathbf{u}}_{\eta}$ based on the second-order ETC (2.10), using stability results (Thm. 2.3). Instead of working directly on the difference $\mathbf{u}_{\eta}^{\mathrm{E}}-\widetilde{\mathbf{u}}_{\eta}$, we apply the well-known technique of using separate asymptotic expansions of $\mathbf{u}_{\eta}^{\mathrm{E}}$ and $\widetilde{\mathbf{u}}_{\eta}$ (see for instance [20], or $[15,16]$ for the electromagnetic case), which are introduced and justified in Sections 4.1 and 4.2 , respectively. Theorem 2.5 is then easily proven, as shown in Section 4.3.

\subsection{Asymptotic expansion of the exact transmission solution}

For a given integer $k \geq 0$, an asymptotic $k$ th order expansion $\mathbf{u}_{\eta}^{(k)}$ of the exact transmission solution $\mathbf{u}_{\eta}$, of the form

$$
\mathbf{u}_{\eta}^{(k)}(\mathbf{x}, t)=\mathbf{u}_{\eta}^{0}(\mathbf{x}, t)+\eta \mathbf{u}_{\eta}^{1}(\mathbf{x}, t)+\ldots+\eta^{k} \mathbf{u}_{\eta}^{k}(\mathbf{x}, t), \quad \text { in } \mathbb{R}^{d} \times \mathbb{R}^{+}
$$

is now introduced. Let us emphasize that that the terms $\mathbf{u}_{\eta}^{k}(\mathbf{x}, t)$ in the expansion (4.1) depend on $\eta$ mainly because their restrictions to $\Omega_{\eta}^{\mathrm{E}}$ and $\Omega_{\eta}^{\mathrm{I}}$, namely $\mathbf{u}_{\eta}^{\ell, \mathrm{E}}$ and $\mathbf{u}_{\eta}^{\ell, \mathrm{I}}$, are defined separately (as explained below) in domains which depend on $\eta$. In some sense they may be treated as independent of $\eta$. This will be formalized with Lemma 4.1. 


\subsubsection{Definition of the restrictions $\mathbf{u}_{\eta}^{\ell, \mathrm{E}}$ of $\mathbf{u}_{\eta}^{\ell}$ in the exterior domain $\Omega_{\eta}^{\mathrm{E}}$}

The $\mathbf{u}_{\eta}^{\ell, E}$ are defined, inductively on $\ell$, as the solution of particular evolution problems. First, each $\mathbf{u}_{\eta}^{\ell, \mathrm{E}}$ satisfies the field equation

$$
\rho \partial_{t}^{2} \mathbf{u}_{\eta}^{\ell, \mathrm{E}}-\operatorname{div} \boldsymbol{\sigma}\left(\mathbf{u}_{\eta}^{\ell, \mathrm{E}}\right)=0, \quad \text { in } \Omega_{\eta}^{\mathrm{E}} \times \mathbb{R}^{+},
$$

and the initial conditions

$$
\begin{array}{llll}
\mathbf{u}_{\eta}^{0, \mathrm{E}}(\cdot, 0)=\mathbf{u}^{0} & \text { and } & \partial_{t} \mathbf{u}_{\eta}^{0, \mathrm{E}}(\cdot, 0)=\mathbf{u}^{1}, \\
\mathbf{u}_{\eta}^{\ell, \mathrm{E}}(\cdot, 0)=0 & \text { and } \quad \partial_{t} \mathbf{u}_{\eta}^{\ell, \mathrm{E}}(\cdot, 0)=0 & (\ell>0),
\end{array}
$$

Then, the transmission conditions for $\mathbf{u}_{\eta}^{\ell, \mathrm{E}}$ are suggested by the relations given in Lemma 3.5, used in such a way that the conditions for $\mathbf{u}_{\eta}^{\ell, \mathrm{E}}$ only involve the $\mathbf{u}_{\eta}^{m \text {, E }}$ for $m \leq \ell$. Hence, for $\ell=0$, the transmission conditions for $\mathbf{u}_{\eta}^{0, \mathrm{E}}$ are simply chosen as

$$
\left[\mathbf{u}_{\eta}^{0, \mathrm{E}}\right]_{\eta}=0, \quad\left[\mathbf{t}\left(\mathbf{u}_{\eta}^{0, \mathrm{E}}\right)\right]_{\eta}=0 \quad \text { in } \Gamma \times \mathbb{R}^{+}
$$

while those for all $\mathbf{u}_{\eta}^{\ell, \mathrm{E}}(\ell \geq 1)$ are chosen as suggested by relations (3.23a) and (3.23b) found in Section 3.2, i.e.:

$$
\begin{aligned}
\left\langle\mathbf{t}\left(\mathbf{u}_{\eta}^{\ell-1, \mathrm{E}}\right)\right\rangle_{\eta} & =\sum_{0 \leq 2 j \leq \ell-1}\left\{\mathcal{C}_{2 j+1}\left\langle\mathbf{u}_{\eta}^{\ell-2 j-1, \mathrm{E}}\right\rangle_{\eta}+\mathcal{D}_{2 j}\left[\mathbf{u}_{\eta}^{\ell-2 j, \mathrm{E}}\right]_{\eta}\right\}, \\
{\left[\mathbf{t}\left(\mathbf{u}_{\eta}^{\ell, \mathrm{E}}\right)\right]_{\eta} } & =\sum_{1 \leq 2 j+1 \leq \ell} 2\left\{\mathcal{C}_{2 j+2}\left\langle\mathbf{u}_{\eta}^{\ell-2 j-1, \mathrm{E}}\right\rangle_{\eta}+\mathcal{D}_{2 j+1}\left[\mathbf{u}_{\eta}^{\ell-2 j, \mathrm{E}}\right]_{\eta}\right\} .
\end{aligned}
$$

The reader will realize that imposing $(4.2 \mathrm{~d})$ and (4.2e) amounts to assigning known values (from the previous steps of the recursion) to the jumps $\left[\mathbf{t}\left(\mathbf{u}_{\eta}^{\ell, \mathrm{E}}\right)\right]_{\eta}$ and $\left[\mathbf{u}_{\eta}^{\ell, \mathrm{E}}\right]_{\eta}$. Indeed, since $\mathcal{D}_{0}=A,(4.2 \mathrm{~d})$ yields

$$
A\left[\mathbf{u}_{\eta}^{\ell, \mathrm{E}}\right]_{\eta}=\left\langle\mathbf{t}\left(\mathbf{u}_{\eta}^{\ell-1, \mathrm{E}}\right)\right\rangle_{\eta}-\sum_{1 \leq 2 j+1 \leq \ell} \mathcal{C}_{2 j+1}\left\langle\mathbf{u}_{\eta}^{\ell-(2 j+1), \mathrm{E}}\right\rangle_{\eta}-\sum_{2 \leq 2 j \leq \ell-1} \mathcal{D}_{2 j}\left[\mathbf{u}_{\eta}^{\ell-2 j, \mathrm{E}}\right]_{\eta} .
$$

where the right hand side involves all $\mathbf{u}_{\eta}^{m, \mathrm{E}}$ for $m<\ell$ while $(4.2 \mathrm{e})$ can be rewritten (since $\mathcal{D}_{1}=\frac{1}{2} \mathcal{B}^{\star}$ ) as

$$
\left[\mathbf{t}\left(\mathbf{u}_{\eta}^{\ell, \mathrm{E}}\right)\right]_{\eta}=\sum_{1 \leq 2 j+1 \leq \ell} 2 \mathcal{C}_{2 j+2}\left\langle\mathbf{u}_{\eta}^{\ell-(2 j+1), \mathrm{E}}\right\rangle_{\eta}+\sum_{2 \leq 2 j \leq \ell-1} 2 \mathcal{D}_{2 j+1}\left[\mathbf{u}_{\eta}^{\ell-2 j, \mathrm{E}}\right]_{\eta}+\mathcal{B}^{\star}\left[\mathbf{u}_{\eta}^{\ell, \mathrm{E}}\right]_{\eta} .
$$

Equations $(4.2 \mathrm{a}),(4.2 \mathrm{~b}),(4.2 \mathrm{f})$ and $(4.2 \mathrm{~g})$ correspond to a standard elastodynamic transmission problem. They define each $\mathbf{u}_{\eta}^{\ell, \mathrm{E}}(\ell>0)$ in $\Omega_{\eta}^{\mathrm{E}}$, recursively in $\ell$, while $\mathbf{u}_{\eta}^{0, \mathrm{E}}$ is defined by (4.2a)-(4.2c) (see also the proof of Lem. 4.1).

Is not difficult, albeit rather tedious, to establish uniform estimates in $\eta$ of the coefficients. In particular, we shall use in the sequel the following estimates:

Lemma 4.1. Assume that (2.21) and (2.24) are satisfied. Then, each function $\mathbf{u}_{\eta}^{\ell, \mathrm{E}}$ is of class $C^{\infty}$ (in space and time) in $\Omega_{\eta, \eta^{\star}} \times \mathbb{R}^{+}$. Moreover, for any $m \in \mathbb{N}$, any multi-index $\alpha \in \mathbb{N}^{d}$ and any $T>0$, there exists a constant $C_{\ell, m, \alpha}^{\mathrm{E}}(T)$, independent of $\eta$ (but depending on the initial data) such that

$$
\forall t \in[0, T], \quad\left\|\partial_{t}^{m} \partial^{\alpha} \mathbf{u}_{\eta}^{\ell, \mathrm{E}}(\cdot, t)\right\|_{L^{2}\left(\Omega_{\eta}^{\mathrm{I}}\right)} \leqslant C_{\ell, m, \alpha}^{\mathrm{E}}(T) .
$$

Proof. We only sketch the proof, whose details are tedious. For $\ell=0$, the regularity result follows from standard regularity theory for second order linear hyperbolic equation (similarly to what is said in Section 2.3, and based 
on the references given therein) and the estimate follows from

- the conservation of energy for both $\mathbf{u}_{\eta}^{\ell, \mathrm{E}}$ itself and its successive time derivatives,

- embeddings similar to those of (2.23), again following from elliptic regularity theory.

For $\ell \geq 1$, we simply note that, up to a trivial translational change of variable $\left(\nu \rightarrow \nu^{\prime}:=\nu \mp \eta\right.$ for $\left.\pm \nu>\eta\right)$ that transforms $\Omega_{\eta}^{\mathrm{E}}$ into $\Omega_{0}, \mathbf{v}_{\eta}\left(\nu^{\prime}, t\right):=\mathbf{u}_{\eta}^{\ell, \mathrm{E}}(\nu, t)$ is the solution on a standard transmission of the form

$$
\left\{\begin{aligned}
\rho \partial_{t}^{2} \mathbf{v}_{\eta}-\operatorname{div} \boldsymbol{\sigma}\left(\mathbf{v}_{\eta}\right)=0, & \text { in } \Omega_{0} \times \mathbb{R}^{+}, \\
{\left[\mathbf{v}_{\eta}\right]_{0}=\mathbf{f}_{\eta}, \quad\left[\mathbf{t}\left(\mathbf{v}_{\eta}\right)\right]_{0}=\mathbf{g}_{\eta} } & \operatorname{across} \Gamma,
\end{aligned}\right.
$$

where $\mathbf{f}_{\eta}$ and $\mathbf{g}_{\eta}$ are given smooth vector fields on $\Gamma \times \mathbb{R}^{+}$. More precisely, according to (4.2f) and (58g), $\mathbf{f}_{\eta}=\mathbf{f}_{\eta}^{\ell}$ and $\mathbf{g}_{\eta}=\mathbf{g}_{\eta}^{\ell}$ where $\mathbf{f}_{\eta}^{\ell}$ and $\mathbf{g}_{\eta}^{\ell}$ are linear combinations of traces of derivatives (up to order $2 \ell$ ) of the $\mathbf{u}_{\eta}^{j, \mathrm{E}}$ for $j \leq \ell-1$. Thus using (4.3) with $\ell$ replaced by $j \leq \ell-1$, together with trace theorems, we easily get, for any $m \geq 0$ and any tangential differential operator $\partial^{\beta}$ along $\Gamma$,:

$$
\left\|\partial_{t}^{m} \partial^{\beta} \mathbf{f}_{\eta}^{\ell}(\cdot, t)\right\|_{L^{2}\left(\Omega_{\eta}^{\mathrm{I}}\right)}+\left\|\partial_{t}^{m} \partial^{\beta} \mathbf{g}_{\eta}^{\ell}(\cdot, t)\right\|_{L^{2}\left(\Omega_{\eta}^{\mathrm{I}}\right)} \leqslant K_{\ell, m, \beta}^{\mathrm{E}}(T) .
$$

The conclusion then follows from standard properties (energy estimates + regularity results) of the transmission problem and invariance properties of Sobolev norms under translation. The details are left to the reader.

\subsubsection{Definition of the restrictions $\mathbf{u}_{\eta}^{\ell, \mathrm{I}}$ of $\mathbf{u}_{\eta}^{\ell}$ in the interior domain $\Omega_{\eta}^{\mathrm{I}}$}

Each restriction $\mathbf{u}_{\eta}^{\ell, \mathrm{I}}$ of $\mathbf{u}_{\eta}^{\ell}$ to $\Omega_{\eta}^{\mathrm{I}}$ is defined as

$$
\mathbf{u}_{\eta}^{\ell, \mathrm{I}}(\mathbf{x}, t)=\mathbf{U}_{\eta}^{\ell}\left(\mathbf{y}, \eta^{-1} \nu, t\right)
$$

where the functions $\mathbf{U}_{\eta}^{\ell}$ are defined by problems (3.10a,b), (3.11a,b) and (3.12a,b) with $\left\{\mathbf{u}^{\ell}\right\}=\left\{\mathbf{u}_{\eta}^{\ell, \mathrm{E}}\right\}_{\eta}$. Hence, $\mathbf{U}_{\eta}^{0}$ and $\mathbf{U}_{\eta}^{1}$ are given by

$$
\mathbf{U}_{\eta}^{0}(\mathbf{y}, s, t)=\left\langle\mathbf{u}_{\eta}^{0, \mathrm{E}}\right\rangle_{\eta}, \quad \mathbf{U}_{\eta}^{1}(\mathbf{y}, s, t)=\left\langle\mathbf{u}_{\eta}^{1, \mathrm{E}}\right\rangle_{\eta}+\left[\mathbf{u}_{\eta}^{1, \mathrm{E}}\right]_{\eta} s
$$

and each $\mathbf{U}_{\eta}^{\ell}(\ell \geq 2)$ then solves system (3.12a) and (3.12b) with boundary data $\left\{\mathbf{u}^{\ell}\right\}=\left\{\mathbf{u}_{\eta}^{\ell, E}\right\}_{\eta}$.

The coefficients $\mathbf{u}_{\eta}^{\ell, \mathrm{I}}$ strongly depend on $\eta$ because of the scaling (4.5). By contrast, the coefficients $\mathbf{U}_{\eta}^{\ell}$ are defined in a fixed domain and depend on $\eta$ only weakly (through the Dirichlet data $\left\{\mathbf{u}_{\eta}^{\ell, E}\right\}_{\eta}$ ). Let us emphasize this by the following lemma:

Lemma 4.2. Assume that (2.21) and (2.24) are satisfied. Then, each function $\mathbf{U}_{\eta}^{\ell}$ is of class $C^{\infty}$ (in space and time) in $\Omega_{1}^{\mathrm{I}} \times \mathbb{R}^{+}$. Moreover, for any $m \in \mathbb{N}$, any multi-index $\alpha \in \mathbb{N}^{d}$ and any $T>0$, there exists a constant $C_{\ell, m, \alpha}^{\mathrm{I}}(T)$, independent of $\eta$ (but depending on the initial data) such that

$$
\forall t \in[0, T], \quad\left\|\partial_{t}^{m} \partial^{\alpha} \mathbf{U}_{\eta}^{\ell}(\cdot, t)\right\|_{L^{2}\left(\Omega_{1}^{\mathrm{I}}\right)} \leqslant C_{\ell, m, \alpha}^{\mathrm{I}}(T) .
$$

Proof. In its principle, the proof of this lemma is similar to that of Lemma 4.1: it is achieved by induction on $\ell$. In short, $t$ being a parameter, each $\mathbf{U}_{\eta}^{\ell,}(\cdot, t)$ is solution of a Dirichlet problem in $\Omega_{1}^{\mathrm{I}}$ for the operator $\partial_{s}^{2}$ whose Dirichlet data are the traces on $\partial \Omega_{\eta}^{\mathrm{I}}$ of $\mathbf{u}_{\eta}^{\ell, \mathrm{E}}(\cdot, t)$ and whose right hand side is a linear combination of derivatives (up to order $2 \ell$ ) of the $\mathbf{U}_{\eta}^{j}$, for $j \leq \ell-1$. One concludes then using the bounds (4.3), trace theorems and simple properties of this Dirichlet problem (use Lem. 3.3 for instance). Once again, we omit the details.

\subsubsection{Equations satisfied by the approximate field $\mathbf{u}_{\eta}^{(k)}$}

By virtue of the above construction of $\mathbf{u}_{\eta}^{(k) \mathrm{E}}$ and $\mathbf{u}_{\eta}^{(k) \mathrm{I}}, \mathbf{u}_{\eta}^{(k)}$ is continuous across the interfaces $\partial \Omega_{\eta}^{ \pm}$, i.e. obeys the kinematic transmission condition

$$
\mathbf{u}_{\eta}^{(k) \mathrm{I}}=\mathbf{u}_{\eta}^{(k) \mathrm{E}} \quad \text { on } \partial \Omega_{\eta}^{\mathrm{E}} .
$$


On the other hand, the corresponding tractions $\mathbf{t}\left(\mathbf{u}_{\eta}^{(k) \mathrm{E}}\right)$ and $\mathbf{t}^{\mathrm{I}}\left(\mathbf{u}_{\eta}^{(k) \mathrm{I}}\right)$ do not match exactly across the interfaces $\partial \Omega_{\eta}^{ \pm}$. More precisely, the following result holds:

Lemma 4.3. The tractions $\mathbf{t}\left(\mathbf{u}_{\eta}^{(k) \mathrm{E}}\right)$ and $\mathbf{t}^{\mathrm{I}}\left(\mathbf{u}_{\eta}^{(k) \mathrm{I}}\right)$ associated to the asymptotic expansion of the transmission solution in the exterior and interior domains satisfy non homogeneous transmission conditions given by

$$
\mathbf{t}\left(\mathbf{u}_{\eta}^{(k) \mathrm{E}}\right)-\mathbf{t}^{\mathrm{I}}\left(\mathbf{u}_{\eta}^{(k) \mathrm{I}}\right)=\eta^{k+1} A \partial_{\nu} \mathbf{u}_{\eta}^{k+1, \mathrm{I}}, \quad \text { on } \partial \Omega_{\eta}^{ \pm} .
$$

Proof. The proof proceeds by establishing separate expressions for $\mathbf{t}\left(\mathbf{u}_{\eta}^{(k) \mathrm{E}}\right)$ and $\mathbf{t}^{\mathrm{I}}\left(\mathbf{u}_{\eta}^{(k) \mathrm{I}}\right)$, and then evaluating the "transmission gap" $\mathbf{t}\left(\mathbf{u}_{\eta}^{(k) \mathrm{E}}\right)-\mathbf{t}^{\mathrm{I}}\left(\mathbf{u}_{\eta}^{(k) \mathrm{I}}\right)$.

(i) Evaluation of $\mathbf{t}\left(\mathbf{u}_{\eta}^{(k) \mathrm{E}}\right)$. First, the tractions $\mathbf{t}\left(\mathbf{u}_{\eta}^{\ell \mathrm{E}}\right)$ on both sides of the layer boundary are computed as $\mathbf{t}\left(\mathbf{u}_{\eta}^{\ell \mathrm{E}}\right)(\mathbf{y}, \pm \eta / 2, t)=\left\langle\mathbf{t}\left(\mathbf{u}_{\eta}^{\ell \mathrm{E}}\right)\right\rangle_{\eta} \pm \frac{1}{2}\left[\mathbf{t}\left(\mathbf{u}_{\eta}^{\ell \mathrm{E}}\right)\right]_{\eta}$, with $\left\langle\mathbf{t}\left(\mathbf{u}_{\eta}^{\ell \mathrm{E}}\right)\right\rangle_{\eta}$ and $\left[\mathbf{t}\left(\mathbf{u}_{\eta}^{\ell \mathrm{E}}\right)\right]_{\eta}$ given by $(4.2 \mathrm{~d})$ and $(4.2 \mathrm{e})$. Performing this calculation (which entails replacing $\ell$ by $\ell+1$ in (4.2d)) and rearranging the resulting combination of sums over even and odd indices into a single sum (by setting $2 j+1=m$ in (4.2d) and $2 j+2=m$ in $(4.2 \mathrm{e}))$, one obtains

$$
\begin{aligned}
\mathbf{t}\left(\mathbf{u}_{\eta}^{\ell, \mathrm{E}}\right)(\mathbf{y}, \pm \eta / 2, t) & =\sum_{1 \leq m \leq \ell+1}( \pm 1)^{m+1}\left\{\mathcal{C}_{m}\left\langle\mathbf{u}_{\eta}^{\ell+1-m, \mathrm{E}}\right\rangle_{\eta}+\mathcal{D}_{m-1}\left[\mathbf{u}_{\eta}^{\ell+2-m, \mathrm{E}}\right]_{\eta}\right\}, \\
& =\sum_{1 \leq m \leq \ell+1}( \pm 1)^{m+1}\left\{\mathcal{C}_{m}\left\langle\mathbf{u}_{\eta}^{\ell+1-m, \mathrm{I}}\right\rangle_{\eta},+\mathcal{D}_{m-1}\left[\mathbf{u}_{\eta}^{\ell+2-m, \mathrm{I}}\right]_{\eta}\right\}
\end{aligned}
$$

where the second equality results from the adopted construction of $\mathbf{u}_{\eta}^{\ell, \mathrm{E}}$ and $\mathbf{u}_{\eta}^{\ell, \mathrm{I}}$. Invoking (4.5) for $\nu= \pm \eta / 2$ and comparing the sum in the last equality above with $\partial_{s} \mathbf{U}^{\ell+1}$ as given by (3.22), one finds

$$
\mathbf{t}_{ \pm}\left(\mathbf{u}_{\eta}^{\ell, \mathrm{E}}\right)\left(\mathbf{y}, \pm \frac{1}{2}, t\right)=A \partial_{s} \mathbf{U}^{\ell+1}\left(\mathbf{y}, \pm \frac{1}{2}, t\right)+\mathcal{B} \mathbf{U}^{\ell}\left(\mathbf{y}, \pm \frac{1}{2}, t\right)=A \partial_{s} \mathbf{u}_{\eta}^{\ell+1, \mathrm{I}}(\mathbf{y}, \pm \eta / 2, t)+\mathcal{B} \mathbf{u}_{\eta}^{\ell, \mathrm{I}}(\mathbf{y}, \pm \eta / 2, t)
$$

(with the last equality stemming from $\mathbf{U}^{\ell}\left(\cdot, \pm \frac{1}{2}, \cdot\right)=\mathbf{u}_{\eta}^{\ell, \mathrm{I}}(\cdot, \pm \eta / 2, \cdot)=\mathbf{u}_{\eta}^{\ell, \mathrm{E}}(\cdot, \pm \eta / 2, \cdot)$ for any $\ell$ by virtue of (4.5) and the assumed kinematic continuity of $\mathbf{u}_{\eta}^{\ell}$ ). On multiplying the above equality by $\eta^{\ell}$, summing the resulting equations over $0 \leq \ell \leq k$ and using that $\partial_{s} \mathbf{U}^{0}=0$ and $\partial_{s} \equiv \eta \partial_{\nu}$, one finally obtains

$$
\mathbf{t}_{ \pm}\left(\mathbf{u}_{\eta}^{(k) \mathrm{E}}\right)\left(\mathbf{y}, \pm \frac{1}{2}, t\right)=A \partial_{\nu} \mathbf{u}_{\eta}^{(k+1) \mathrm{I}}\left(\mathbf{y}, \pm \frac{1}{2}, t\right)+\mathcal{B} \mathbf{u}_{\eta}^{(k) \mathrm{I}}\left(\mathbf{y}, \pm \frac{1}{2}, t\right)
$$

(ii) Evaluation of $\mathbf{t}^{\mathrm{I}}\left(\mathbf{u}_{\eta}^{(k) \mathrm{I}}\right)$. The tractions $\mathbf{t}^{\mathrm{I}}\left(\mathbf{u}_{\eta}^{\ell, \mathrm{I}}\right)$ are obtained by applying (3.8) with $\mathbf{u}=\mathbf{u}_{\eta}^{\ell, \mathrm{I}}=\mathbf{U}^{\ell}$, i.e.:

$$
\mathbf{t}_{ \pm}^{\mathrm{I}}\left(\mathbf{u}_{\eta}^{\ell, \mathrm{I}}\right)\left(\mathbf{y}, \pm \frac{1}{2}, t\right)=\eta^{-1} A \partial_{s} \mathbf{U}^{\ell}\left(\mathbf{y}, \pm \frac{1}{2}, t\right)+\mathcal{B} \mathbf{U}^{\ell}\left(\mathbf{y}, \pm \frac{1}{2}, t\right)
$$

On multiplying the above equality by $\eta^{\ell}$, summing the resulting equations over $0 \leq \ell \leq k$ and invoking ansatz (4.1), one finds

$$
\mathbf{t}_{ \pm}^{\mathrm{I}}\left(\mathbf{u}_{\eta}^{(k) \mathrm{I}}\right)\left(\mathbf{y}, \pm \frac{1}{2}, t\right)=A \partial_{\nu} \mathbf{u}_{\eta}^{(k) \mathrm{I}}\left(\mathbf{y}, \pm \frac{1}{2}, t\right)+\mathcal{B} \mathbf{u}_{\eta}^{(k) \mathrm{I}}\left(\mathbf{y}, \pm \frac{1}{2}, t\right)
$$

(iii) Evaluation of the transmission gap $\mathbf{t}\left(\mathbf{u}_{\eta}^{(k) \mathrm{E}}\right)-\mathbf{t}^{\mathrm{I}}\left(\mathbf{u}_{\eta}^{(k) \mathrm{I}}\right)$. The desired result (4.8) finally follows directly by subtracting (4.9b) from (4.9a) and recalling ansatz (4.1).

Lemma 4.4. Assume that (2.21) and (2.24) are satisfied. Then, for any $k \in \mathbb{N}$ and $T>0$, there exists a constant $C_{k}(T)$, independent of $\eta$ (but depending on the material properties and the initial data) such that

$$
\forall t \in[0, T], \quad\left\|\left(\partial_{t} \mathbf{u}_{\eta}-\partial_{t} \mathbf{u}_{\eta}^{(k)}\right)(\cdot, t)\right\|_{L^{2}\left(\Omega_{\eta}^{\mathrm{E}}\right)}+\left\|\left(\mathbf{u}_{\eta}-\mathbf{u}_{\eta}^{(k)}\right)(\cdot, t)\right\|_{H^{1}\left(\Omega_{\eta}^{\mathrm{E}}\right)} \leqslant C_{k}(T) \eta^{k+1} .
$$

Proof. The proof consists of computing (Step 2) and estimating (Step 3) the energy associated to the error field

$$
\mathbf{e}_{\eta}^{(k)}:=\mathbf{u}_{\eta}-\mathbf{u}_{\eta}^{(k)},
$$


to obtain a control over $\left\|\partial_{t} \mathbf{e}_{\eta}^{(k)}\right\|_{L^{2}\left(\Omega_{\eta}^{\mathrm{E}}\right)}$ and $\left\|\mathbf{e}_{\eta}^{(k)}\right\|_{H^{1}\left(\Omega_{\eta}^{\mathrm{E}}\right)}$. It is first necessary, as a preparatory move, to determine the governing equations satisfied by the error field (Step 1).

Step 1. Governing equations for $\mathbf{e}_{\eta}^{(k)}$. In $\Omega_{\eta}^{\mathrm{E}}$, we have by construction

$$
\rho \partial_{t}^{2} \mathbf{u}_{\eta}^{(k) \mathrm{E}}-\operatorname{div} \boldsymbol{\sigma}\left(\mathbf{u}_{\eta}^{(k) \mathrm{E}}\right)=0 .
$$

Besides, the equation satisfied by $\mathbf{u}_{\eta}^{(k) \mathrm{I}}$ inside $\Omega_{\eta}^{\mathrm{I}}$ is found, by summing equation (3.10a), equation (3.11a) weighted by $\eta$, and equations $(3.12 \mathrm{a})_{m}$ weighted by $\eta^{m}(2 \leq m \leq k+1)$ to be

$$
A \partial_{s}^{2}\left(\sum_{m=0}^{k+1} \eta^{m} \mathbf{U}^{m}\right)=\left(\rho^{\mathrm{I}} \partial_{t}^{2}-\mathcal{P}_{2}\right)\left(\sum_{m=2}^{k+1} \eta^{m} \mathbf{U}^{m-2}\right)-\partial_{s} \mathcal{P}_{1}\left(\sum_{m=1}^{k+1} \eta^{m} \mathbf{U}^{m-1}\right)
$$

After reverting to the original space coordinates $(\mathbf{y}, \nu)$, this yields (using $\partial_{s} \equiv \eta \partial_{\nu}$ )

$$
\eta^{2} A \partial_{\nu}^{2}\left(\sum_{m=0}^{k+1} \eta^{m} \mathbf{u}_{\eta}^{m, \mathrm{I}}\right)=\left(\rho^{\mathrm{I}} \partial_{t}^{2}-\mathcal{P}_{2}\right)\left(\sum_{m=2}^{k+1} \eta^{m} \mathbf{u}_{\eta}^{m-2, \mathrm{I}}\right)-\eta \partial_{\nu} \mathcal{P}_{1}\left(\sum_{m=1}^{k+1} \eta^{m} \mathbf{u}_{\eta}^{m-1, \mathrm{I}}\right)
$$

which is rewritten, after a change of summation index in the last two sums, as

$$
\eta^{2} A \partial_{\nu}^{2}\left(\sum_{m=0}^{k+1} \eta^{m} \mathbf{u}_{\eta}^{m, \mathrm{I}}\right)=\eta^{2}\left(\rho^{\mathrm{I}} \partial_{t}^{2}-\mathcal{P}_{2}\right)\left(\sum_{m=0}^{k-1} \eta^{m} \mathbf{u}_{\eta}^{m, \mathrm{I}}\right)-\eta^{2} \partial_{\nu} \mathcal{P}_{1}\left(\sum_{m=0}^{k} \eta^{m} \mathbf{u}_{\eta}^{m, \mathrm{I}}\right)
$$

Dropping the $\eta^{2}$ factor and making $\mathbf{u}_{\eta}^{(k) \mathrm{I}}$ appear, we obtain

$$
A \partial_{\nu}^{2}\left(\mathbf{u}_{\eta}^{(k) \mathrm{I}}+\eta^{k+1} \mathbf{u}_{\eta}^{k+1, \mathrm{I}}\right)=\left(\rho^{\mathrm{I}} \partial_{t}^{2}-\mathcal{P}_{2}\right)\left(\mathbf{u}_{\eta}^{(k) \mathrm{I}}-\eta^{k} \mathbf{u}_{\eta}^{k, \mathrm{I}}\right)-\partial_{\nu} \mathcal{P}_{1} \mathbf{u}_{\eta}^{(k) \mathrm{I}}
$$

which can be rewritten, using (3.6) (with $\mathbf{u}_{\eta}^{(k) \mathrm{I}}$ instead of $\mathbf{u}_{\eta}^{\mathrm{I}}$ ) as

$$
\rho^{\mathrm{I}} \partial_{t}^{2} \mathbf{u}_{\eta}^{(k) \mathrm{I}}-\operatorname{div} \boldsymbol{\sigma}^{\mathrm{I}}\left(\mathbf{u}_{\eta}^{(k) \mathrm{I}}\right)=\eta^{k+1} A \partial_{\nu}^{2} \mathbf{u}_{\eta}^{k+1, \mathrm{I}}+\eta^{k}\left(\rho^{\mathrm{I}} \partial_{t}^{2}-\mathcal{P}_{2}\right) \mathbf{u}_{\eta}^{k, \mathrm{I}} \quad \text { in } \Omega_{\eta}^{\mathrm{I}} .
$$

Recalling that $\mathbf{u}_{\eta}$ solves the homogeneous elastodynamic field equation (2.3) in $\mathbb{R}^{d}$, and in view of the initial conditions (4.2b) and transmission conditions (4.7) and (4.8), one concludes that the error field satisfies

$$
\begin{aligned}
& \rho \partial_{t}^{2} \mathbf{e}_{\eta}^{(k) \mathrm{E}}-\operatorname{div} \boldsymbol{\sigma}^{\mathrm{E}}\left(\mathbf{e}_{\eta}^{(k) \mathrm{E}}\right)=0 \quad \text { in } \Omega_{\eta}^{\mathrm{E}} \times \mathbb{R}^{+} \\
& \rho^{\mathrm{I}} \partial_{t}^{2} \mathbf{e}_{\eta}^{(k) \mathrm{I}}-\operatorname{div} \boldsymbol{\sigma}^{\mathrm{I}}\left(\mathbf{e}_{\eta}^{(k) \mathrm{I}}\right)=\eta^{k+1} A \partial_{\nu}^{2} \mathbf{u}_{\eta}^{k+1, \mathrm{I}}+\eta^{k}\left(\rho \partial_{t}^{2}-\mathcal{P}_{2}\right) \mathbf{u}_{\eta}^{k, \mathrm{I}} \quad \text { in } \Omega_{\eta}^{\mathrm{I}} \times \mathbb{R}^{+} \\
& \mathbf{e}_{\eta}^{(k) \mathrm{E}}-\mathbf{e}_{\eta}^{(k) \mathrm{I}}=0, \quad \mathbf{t}\left(\mathbf{u}_{\eta}^{(k) \mathrm{E}}\right)-\mathbf{t}^{\mathrm{I}}\left(\mathbf{u}_{\eta}^{(k) \mathrm{I}}\right)=\eta^{k+1} A \partial_{\nu} \mathbf{u}_{\eta}^{k+1, \mathrm{I}} \quad \text { on } \partial \Omega_{\eta}^{ \pm} \times \mathbb{R}^{+} \\
& \begin{array}{ll}
\mathbf{e}_{\eta}^{(k)}(\cdot, 0)=\partial_{t} \mathbf{e}_{\eta}^{(k)}(\cdot, 0)=0 & \text { in } \mathbb{R}^{d}
\end{array}
\end{aligned}
$$

At first glance, one expects that $\mathbf{e}_{\eta}^{(k) \mathrm{E}}$ is $O\left(\eta^{k}\right)$ since the volume source term has a $O\left(\eta^{k}\right)$ contribution (whose coefficient is only weakly dependent on $\eta$ ). This too-simple reasoning disregards the fact that the support $\Omega_{\eta}^{\mathrm{I}}$ of the source term is of small thickness, which will result in an $O\left(\eta^{k+1}\right)$ energy estimate.

Step 2. Energy of $\mathbf{e}_{\eta}^{(k)}$. From now on, to any smooth enough time dependent vector field $\mathbf{v}$, we associate the elastodynamic energy $\mathcal{E}_{\eta}(\mathbf{v}, t)$ given (recalling Defs. $(2.14,2.15)$ of $\rho_{\eta}$ and $\left.\boldsymbol{\sigma}_{\eta}(\cdot)\right)$ by:

$$
\mathcal{E}_{\eta}(\mathbf{v}, t)=\int_{\mathbb{R}^{d}}\left(\frac{\rho_{\eta}}{2}\left|\partial_{t} \mathbf{v}\right|^{2}+\frac{1}{2} \sigma_{\eta}(\mathbf{v}): \varepsilon(\mathbf{v})\right) \mathrm{d} \mathbf{x}
$$


Accordingly, the elastodynamic energy $\mathcal{E}_{\eta}^{k}(t)$ of the error field is given by

$$
\mathcal{E}_{\eta}^{k}(t):=\mathcal{E}_{\eta}\left(\mathbf{e}_{\eta}^{(k)}, t\right)=\left\{\int_{\Omega_{\eta}^{+}}+\int_{\Omega_{\eta}^{-}}+\int_{\Omega_{\eta}^{\mathrm{I}}}\right\}\left(\frac{\rho_{\eta}}{2}\left|\partial_{t} \mathbf{e}_{\eta}^{(k)}\right|^{2}+\frac{1}{2} \boldsymbol{\sigma}_{\eta}\left(\mathbf{e}_{\eta}^{(k)}\right): \varepsilon\left(\mathbf{e}_{\eta}^{(k)}\right)\right) \mathrm{d} \mathbf{x} .
$$

Therefore, differentiating $\mathcal{E}(t)$ and using Green's formula, we obtain

$$
\begin{aligned}
\frac{\mathrm{d} \mathcal{E}_{\eta}^{k}}{\mathrm{~d} t}= & \int_{\mathbb{R}^{d}} \\
& \left(\rho_{\eta} \partial_{t}^{2} \mathbf{e}_{\eta}^{(k)}-\operatorname{div} \boldsymbol{\sigma}_{\eta}\left(\mathbf{e}_{\eta}^{(k)}\right)\right) \cdot \partial_{t} \mathbf{e}_{\eta}^{(k)} \mathrm{d} \mathbf{x} \\
& \quad \int_{\partial \Omega_{\eta}^{+}}\left(\mathbf{t}^{\mathrm{I}}\left(\mathbf{e}_{\eta}^{(k) \mathrm{I}}\right)-\mathbf{t}\left(\mathbf{e}_{\eta}^{(k) \mathrm{E}}\right) \cdot \partial_{t} \mathbf{e}_{\eta}^{(k)} \mathrm{d} \mathbf{y}-\int_{\partial \Omega_{\eta}^{-}}\left(\mathbf{t}^{\mathrm{I}}\left(\mathbf{e}_{\eta}^{(k) \mathrm{I}}\right)-\mathbf{t}\left(\mathbf{e}_{\eta}^{(k) \mathrm{E}}\right) \cdot \partial_{t} \mathbf{e}_{\eta}^{(k)} \mathrm{d} \mathbf{y}\right.\right. \\
= & \int_{\Omega_{\eta}^{\mathrm{I}}}\left(\eta^{k+1} A \partial_{\nu}^{2} \mathbf{u}_{\eta}^{k+1, \mathrm{I}}+\eta^{k}\left(\rho^{\mathrm{I}} \partial_{t}^{2}-\mathcal{P}_{2}\right) \mathbf{u}_{\eta}^{k, \mathrm{I}}\right) \cdot \partial_{t} \mathbf{e}_{\eta}^{(k)} \mathrm{d} \mathbf{x} \\
& \quad-\int_{\partial \Omega_{\eta}^{+}} \eta^{k+1} A \partial_{\nu} \mathbf{u}_{\eta}^{k+1, \mathrm{I}} \cdot \partial_{t} \mathbf{e}_{\eta}^{(k)} \mathrm{d} \mathbf{y}+\int_{\partial \Omega_{\eta}^{-}} \eta^{k+1} A \partial_{\nu} \mathbf{u}_{\eta}^{k+1, \mathrm{I}} \cdot \partial_{t} \mathbf{e}_{\eta}^{(k)} \mathrm{d} \mathbf{y}
\end{aligned}
$$

where the last equality uses equations (4.10). The idea now is to estimate the right hand side for the above equality in terms of the energy $\mathcal{E}_{\eta}^{k}(t)$ itself, in order to conclude using Gronwall-type inequalities. A difficulty lies in the fact that the traces on $\partial \Omega_{\eta}^{ \pm}$of $\partial_{t} \mathbf{e}_{\eta}^{(k)}$ can not be estimated, in $L^{2}(\Gamma)$-norms for instance, in terms of $\mathcal{E}_{\eta}^{k}(t)$. However, these traces can be eliminated using the equality

$$
\int_{\Omega_{\eta}^{\mathrm{I}}} A \partial_{\nu}^{2} \mathbf{u}_{\eta}^{k+1, \mathrm{I}} \cdot \partial_{t} \mathbf{e}_{\eta}^{(k)} \mathrm{d} \mathbf{x}-\int_{\partial \Omega_{\eta}^{+}} A \partial_{\nu} \mathbf{u}_{\eta}^{k+1, \mathrm{I}} \cdot \partial_{t} \mathbf{e}_{\eta}^{(k)} \mathrm{d} \mathbf{y}+\int_{\partial \Omega_{\eta}^{-}} A \partial_{\nu} \mathbf{u}_{\eta}^{k+1, \mathrm{I}} \cdot \partial_{t} \mathbf{e}_{\eta}^{(k)} \mathrm{d} \mathbf{y}=-\int_{\Omega_{\eta}^{\mathrm{I}}} A \partial_{\nu} \mathbf{u}_{\eta}^{k+1, \mathrm{I}} \cdot \partial_{t} \partial_{\nu} \mathbf{e}_{\eta}^{(k)} \mathrm{d} \mathbf{x},
$$

obtained via an integration by parts in the variable $\nu$, so that we get the new identity

$$
\frac{\mathrm{d} \mathcal{E}_{\eta}^{k}}{\mathrm{~d} t}=\eta^{k} \int_{\Omega_{\eta}^{\mathrm{I}}}\left(\rho^{\mathrm{I}} \partial_{t}^{2}-\mathcal{P}_{2}\right) \mathbf{u}_{\eta}^{k, \mathrm{I}} \cdot \partial_{t} \mathbf{e}_{\eta}^{(k)} \mathrm{d} \mathbf{x}-\eta^{k+1} \int_{\Omega_{\eta}^{\mathrm{I}}} A \partial_{\nu} \mathbf{u}_{\eta}^{k+1, \mathrm{I}} \cdot \partial_{t} \partial_{\nu} \mathbf{e}_{\eta}^{(k)} \mathrm{d} \mathbf{x} .
$$

By doing so, the previous difficulty has been replaced by another: the term $\partial_{t} \partial_{\nu} \mathbf{e}_{\eta}^{(k)}$ can not be estimated, in the $L^{2}\left(\Omega_{\eta}^{\mathrm{I}}\right)$-norm for instance, with the energy. Instead, we use a different trick which exploits an integration by parts in time, allowing to "replace" $\partial_{t} \partial_{\nu} \mathbf{e}_{\eta}^{(k)}$ by $\partial_{\nu} \mathbf{e}_{\eta}^{(k)}$ which can then be estimated using the energy. More precisely, since

$$
\int_{0}^{t} \int_{\Omega_{\eta}^{\mathrm{I}}} A \partial_{\nu} \mathbf{u}_{\eta}^{k+1, \mathrm{I}} \cdot \partial_{t} \partial_{\nu} \mathbf{e}_{\eta}^{(k)} \mathrm{d} \mathbf{x} \mathrm{d} \tau=-\int_{0}^{t} \int_{\Omega_{\eta}^{\mathrm{I}}} A \partial_{t} \partial_{\nu} \mathbf{u}_{\eta}^{k+1, \mathrm{I}} \cdot \partial_{\nu} \mathbf{e}_{\eta}^{(k)} \mathrm{d} \mathbf{x} \mathrm{d} \tau+\left[\int_{\Omega_{\eta}^{\mathrm{I}}} A \partial_{\nu} \mathbf{u}_{\eta}^{k+1, \mathrm{I}} \cdot \partial_{\nu} \mathbf{e}_{\eta}^{(k)} \mathrm{d} \mathbf{x}\right]_{0}^{t},
$$

and noting that $\mathbf{e}_{\eta}^{(k) \mathrm{I}}$ has homogeneous initial conditions (implying $\left.\mathcal{E}_{\eta}^{k}(0)=0\right), \mathcal{E}_{\eta}^{k}(t)$ is found to be given by

$$
\begin{aligned}
\mathcal{E}_{\eta}^{k}(t)= & \eta^{k} \int_{0}^{t} \int_{\Omega_{\eta}^{\mathrm{I}}}\left(\rho^{\mathrm{I}} \partial_{t}^{2}-\mathcal{P}_{2}\right) \mathbf{u}_{\eta}^{k, \mathrm{I}} \cdot \partial_{t} \mathbf{e}_{\eta}^{(k)} \mathrm{d} \mathbf{x} \mathrm{d} \tau \\
& +\eta^{k+1} \int_{0}^{t} \int_{\Omega_{\eta}^{\mathrm{I}}} A \partial_{t} \partial_{\nu} \mathbf{u}_{\eta}^{k+1, \mathrm{I}} \cdot \partial_{\nu} \mathbf{e}_{\eta}^{(k)} \mathrm{d} \mathbf{x} \mathrm{d} \tau-\eta^{k+1} \int_{\Omega_{\eta}^{\mathrm{I}}} A\left[\partial_{t} \partial_{\nu} \mathbf{u}_{\eta}^{k+1, \mathrm{I}} \cdot \partial_{\nu} \mathbf{e}_{\eta}^{(k)}\right](\cdot, t) \mathrm{d} \mathbf{x}
\end{aligned}
$$

Step 3. $O\left(\eta^{2 k+1}\right)$ estimation of $\mathcal{E}_{\eta}^{k}(t)$. This step consists in determining a uniform upper bound for $\mathcal{E}_{\eta}^{k}(t)$. In the sequel, to avoid notational inflation, we shall use the following conventions:

- $C$ denotes any positive constant whose value may vary from one line to another one but which only depends on the material properties of the propagation medium; 
- $C_{k}(T)$ denotes any positive constant whose value may vary from one line to another one and which depends on the material properties of the propagation medium, the initial data, $k$ and $T$.

We begin by estimating the various terms in the right hand side of (4.14). The first ingredient consists of deriving estimates that exploit the smallness of the domain $\Omega_{\eta}^{\mathrm{I}}$. From (4.5) we easily infer that

$$
\left(\rho^{\mathrm{I}} \partial_{t}^{2}-\mathcal{P}_{2}\right) \mathbf{u}_{\eta}^{k, \mathrm{I}}(\mathbf{y}, \nu, \tau)=\left(\rho^{\mathrm{I}} \partial_{t}^{2}-\mathcal{P}_{2}\right) \mathbf{U}_{\eta}^{k}\left(\mathbf{y}, \eta^{-1} \nu, \tau\right)
$$

which yields immediately, for any $\tau \leq T$

$$
\left\|\left(\rho^{\mathrm{I}} \partial_{t}^{2}-\mathcal{P}_{2}\right) \mathbf{u}_{\eta}^{k, \mathrm{I}}(\cdot, \tau)\right\|_{L^{2}\left(\Omega_{\eta}^{\mathrm{I}}\right)}=\eta^{1 / 2}\left\|\left(\rho^{\mathrm{I}} \partial_{t}^{2}-\mathcal{P}_{2}\right) \mathbf{U}_{\eta}^{k}(\cdot, \tau)\right\|_{L^{2}\left(\Omega_{1}^{\mathrm{I}}\right)} \leq \eta^{1 / 2} C_{k}(T)
$$

where, for the last inequality, we used Lemma 4.2. Proceeding in the same manner, we also obtain for any $\tau \leq T$

$$
\left\|\partial_{t} \partial_{\nu} \mathbf{u}_{\eta}^{k+1, \mathrm{I}}(\cdot, \tau)\right\|_{L^{2}\left(\Omega_{\eta}^{\mathrm{I}}\right)}=\eta^{-1 / 2}\left\|A \partial_{t} \partial_{s} \mathbf{U}_{\eta}^{k+1}(\cdot, \tau)\right\|_{L^{2}\left(\Omega_{1}^{\mathrm{I}}\right)} \leq \eta^{-1 / 2} C_{k}(T) .
$$

Since, by definition of the energy and Korn's inequality ([19], Thm. 10.2), we have

$$
\left\|\partial_{t} \mathbf{e}_{\eta}^{(k)}(\cdot, \tau)\right\|_{L^{2}\left(\Omega_{\eta}^{\mathrm{I}}\right)}+\left\|\partial_{\nu} \mathbf{e}_{\eta}^{(k)}(\cdot, \tau)\right\|_{L^{2}\left(\Omega_{\eta}^{\mathrm{I}}\right)} \leq C \mathcal{E}_{\eta}^{k}(\tau)^{1 / 2}
$$

we deduce from (4.14), (4.15) and (4.16) and the Cauchy-Schwartz inequality that

$$
\mathcal{E}_{\eta}^{k}(t) \leq \eta^{k+1 / 2} C_{k}(T)\left(\int_{0}^{t} \mathcal{E}_{\eta}^{k}(\tau)^{1 / 2} \mathrm{~d} \tau+\mathcal{E}_{\eta}^{k}(t)^{1 / 2}\right)
$$

which yields, by Young's inequality:

$$
\mathcal{E}_{\eta}^{k}(t) \leq \eta^{k+1 / 2} C_{k}(T) \int_{0}^{t} \mathcal{E}_{\eta}^{k}(\tau)^{1 / 2} \mathrm{~d} \tau+\eta^{2 k+1} C_{k}(T) .
$$

Then, a generalization of Gronwall's lemma, known as a Gronwall-Bellman-Bihari inequality ([4], Sect. 3), implies

$$
\mathcal{E}_{\eta}^{k}(t) \leq \eta^{2 k+1} C_{k}(T)
$$

Step 4. $O\left(\eta^{2 k+2}\right)$ estimate of $\mathcal{E}_{\eta}^{k}(t)$. We next claim that, as formally expected

$$
\mathcal{E}_{\eta}^{k}(t)^{1 / 2} \leq C_{k}(T) \eta^{k+1}
$$

From the previous step and from triangular inequality, we deduce that

$$
\mathbf{e}_{\eta}^{(k)}=\mathbf{e}_{\eta}^{(k+1)}-\eta^{k+1} \mathbf{u}_{\eta}^{k+1} \Longrightarrow \mathcal{E}_{\eta}^{k}(t)^{1 / 2} \leq \mathcal{E}_{\eta}^{k+1}(t)^{1 / 2}+\eta^{k+1} \mathcal{E}_{\eta}\left(\mathbf{u}_{\eta}^{k+1}, t\right)^{1 / 2} .
$$

The conclusion follows after having observed that, according to Lemmas 4.1 and 4.2,

$$
\mathcal{E}_{\eta}\left(\mathbf{u}_{\eta}^{k+1}, t\right)^{1 / 2} \leq C_{k}(T) .
$$

Lemma 4.4 finally follows from Korn's inequality. 


\subsection{Asymptotic expansion of the effective transmission solution}

We now introduce, for a given integer $k \geq 0$, an asymptotic expansion $\widetilde{\mathbf{u}}_{\eta}^{(k)}$ of the solution $\widetilde{\mathbf{u}}_{\eta}$ of problem $(2.3,2.4 \mathrm{a}, 2.10)$ through the ansatz

$$
\widetilde{\mathbf{u}}_{\eta}^{(k)}(\mathbf{x}, t)=\widetilde{\mathbf{u}}_{\eta}^{0}(\mathbf{x}, t)+\eta \widetilde{\mathbf{u}}_{\eta}^{1}(\mathbf{x}, t)+\ldots+\eta^{k} \widetilde{\mathbf{u}}_{\eta}^{k}(\mathbf{x}, t), \quad \text { in } \Omega_{\eta}^{\mathrm{E}} \times \mathbb{R}^{+},
$$

where each $\widetilde{\mathbf{u}}_{\eta}^{\ell}$ satisfies the field equation (4.2a) and initial conditions (4.2b). Injecting the ansatz (4.20) into the second-order ETC (2.10), the boundary conditions

$$
\text { (a) } A\left[\widetilde{\mathbf{u}}_{\eta}^{0}\right]_{\eta}=0, \quad \text { (b) }\left[\mathbf{t}\left(\widetilde{\mathbf{u}}_{\eta}^{0}\right)\right]_{\eta}=0
$$

are readily found to permit cancellation of the lowest-order contributions arising in (2.10) (whose order is $O(1)$ for $(2.10 \mathrm{a})$ and $O(\eta)$ for $(2.10 \mathrm{~b}))$. Then, recursively for any $\ell \geq 1$, the boundary conditions

$$
\begin{aligned}
\ell \geq 1: & \text { (a) } r & \left\langle\mathbf{t}\left(\widetilde{\mathbf{u}}_{\eta}^{\ell-1}\right)\right\rangle_{\eta} & =A\left[\widetilde{\mathbf{u}}_{\eta}^{\ell}\right]_{\eta}+\mathcal{B}\left\langle\widetilde{\mathbf{u}}_{\eta}^{\ell-1}\right\rangle_{\eta}, \\
& \text { (b) } & {\left[\mathbf{t}\left(\widetilde{\mathbf{u}}_{\eta}^{\ell}\right)\right]_{\eta} } & =\left(\rho^{\mathrm{I}} \partial_{t}^{2}-\mathcal{P}_{2}\right)\left\langle\widetilde{\mathbf{u}}_{\eta}^{\ell-1}\right\rangle_{\eta}+\mathcal{B}^{\star}\left[\widetilde{\mathbf{u}}_{\eta}^{\ell}\right]_{\eta} .
\end{aligned}
$$

achieve cancellation of the subsequent contributions (of order $O\left(\eta^{\ell}\right)$ for $(2.10 \mathrm{a})$ and $O\left(\eta^{\ell+1}\right)$ for (2.10b)). We then let each $\widetilde{\mathbf{u}}_{\eta}^{\ell}$ in expansion (4.20) to be defined by equations ((2.3)) and (2.4b) and boundary conditions (4.21) (for $\ell=0$ ) or (4.22) (for $\ell \geq 1$ ). More precisely, like for the $\mathbf{u}_{\eta}^{\ell}$ (see (4.2f) and (4.2g)), the $\widetilde{\mathbf{u}}_{\eta}^{\ell}$ are defined recursively on $\ell$. Moreover, like the $\mathbf{u}_{\eta}^{\ell}$, the $\widetilde{\mathbf{u}}_{\eta}^{\ell}$ are "essentially independent" of $\eta$ (they merely depend on $\ell$ through the geometry of $\Omega_{\eta}^{\mathrm{E}}$ ). In particular, we have the following lemma which is the counterpart for the $\widetilde{\mathbf{u}}_{\eta}^{\ell}$ of Lemma 4.1 for the $\mathbf{u}_{\eta}^{\ell}$ :

Lemma 4.5. Assume that (2.21) and (2.24) are satisfied. Then, each function $\mathbf{u}_{\eta}^{\ell, \mathrm{E}}$ is of class $C^{\infty}$ (in space and time) in $\Omega_{\eta, \eta^{\star}} \times \mathbb{R}^{+}$. Moreover, for any $m \in \mathbb{N}$, any multi-index $\alpha \in \mathbb{N}^{d}$ and any $T>0$, there exists a constant $\widetilde{C}_{\ell, m, \alpha}^{\mathrm{E}}(T)$, independent of $\eta$ (but depending on the initial data) such that

$$
\forall t \in[0, T], \quad\left\|\partial_{t}^{m} \partial^{\alpha} \widetilde{\mathbf{u}}_{\eta}^{\ell,}(\cdot, t)\right\|_{L^{2}\left(\Omega_{\eta}^{\mathrm{I}}\right)} \leqslant \widetilde{C}_{\ell, m, \alpha}^{\mathrm{E}}(T)
$$

Now, we multiply equations (4.22a) by $\eta^{\ell}$, sum the resulting equalities for $1 \leq \ell \leq k$ and add equation (4.21a). This, and similar manipulations using equations (4.22b) and (4.21b), yields the relations

$$
\begin{aligned}
\left\langle\mathbf{t}\left(\widetilde{\mathbf{u}}_{\eta}^{(k)}\right)\right\rangle_{\eta} & =\eta^{-1} A\left[\widetilde{\mathbf{u}}_{\eta}^{(k+1)}\right]_{\eta}+\mathcal{B}\left\langle\widetilde{\mathbf{u}}_{\eta}^{(k)}\right\rangle_{\eta}, \\
{\left[\mathbf{t}\left(\widetilde{\mathbf{u}}_{\eta}^{(k)}\right]_{\eta}\right.} & =\eta\left(\rho^{\mathrm{I}} \partial_{t}^{2}-\mathcal{P}_{2}\right)\left\langle\widetilde{\mathbf{u}}_{\eta}^{(k-1)}\right\rangle_{\eta}+\mathcal{B}^{\star}\left[\widetilde{\mathbf{u}}_{\eta}^{k}\right]_{\eta} .
\end{aligned}
$$

Remark 4.6. Conditions (4.21) and (4.2c) are identical, and conditions (4.22) for $\ell=1,2$ are respectively identical to conditions (3.21a) and (3.21b) for $\ell=1,2$. Consequently, expansions (4.1) and (4.20) coincide up to order $k=2$ included.

We now justify expansion (4.20) as an approximation of the effective transmission solution $\widetilde{\mathbf{u}}_{\eta}^{\mathrm{E}}$ :

Lemma 4.7. Assume that (2.21), (2.24) are satisfied. Then, for any $k \in \mathbb{N}$ and $T>0$, there exists a constant $\widetilde{C}_{k}(T)$, independent of $\eta$ (but depending on the material properties of the propagation medium and the initial data) such that

$$
\forall t \in[0, T], \quad\left\|\left(\partial_{t} \widetilde{\mathbf{u}}_{\eta}-\partial_{t} \widetilde{\mathbf{u}}_{\eta}^{(k)}\right)(\cdot, t)\right\|_{L^{2}\left(\Omega_{\eta}^{\mathrm{E}}\right)}+\left\|\left(\widetilde{\mathbf{u}}_{\eta}-\widetilde{\mathbf{u}}_{\eta}^{(k)}\right)(\cdot, t)\right\|_{H^{1}\left(\Omega_{\eta}^{\mathrm{E}}\right)} \leqslant \widetilde{C}_{k}(T) \eta^{k+1} .
$$


Proof. The proof follows the same steps as that of Lemma 4.4.

Step 1. governing equations for the error field $\widetilde{\mathbf{e}}_{\eta}^{(k)}:=\widetilde{\mathbf{u}}_{\eta}-\widetilde{\mathbf{u}}_{\eta}^{(k)}$. On subtracting relations (4.24) from conditions (4.22) and rearranging terms, the error field is found to satisfy the following non-homogeneous boundary conditions

$$
\begin{aligned}
\left\langle\mathbf{t}\left(\widetilde{\mathbf{e}}_{\eta}^{(k)}\right)\right\rangle_{\eta} & =\eta^{-1} A\left[\widetilde{\mathbf{e}}_{\eta}^{(k)}\right]_{\eta}+\mathcal{B}\left\langle\widetilde{\mathbf{e}}_{\eta}^{(k)}\right\rangle_{\eta}-\eta^{k} A\left[\widetilde{\mathbf{u}}_{\eta}^{k+1}\right]_{\eta}, \\
{\left[\mathbf{t}\left(\widetilde{\mathbf{e}}_{\eta}^{(k)}\right]_{\eta}\right.} & =\eta\left(\rho^{\mathrm{I}} \partial_{t}^{2}-\mathcal{P}_{2}\right)\left\langle\widetilde{\mathbf{e}}_{\eta}^{(k)}\right\rangle_{\eta}+\mathcal{B}^{\star}\left[\widetilde{\mathbf{e}}_{\eta}^{k}\right]_{\eta}+\eta^{k+1}\left(\rho^{\mathrm{I}} \partial_{t}^{2}-\mathcal{P}_{2}\right)\left\langle\widetilde{\mathbf{u}}_{\eta}^{k}\right\rangle_{\eta} .
\end{aligned}
$$

whose homogeneous counterpart is seen to coincide with the ETC (2.10). The error field $\widetilde{\mathbf{e}}_{\eta}^{(k)}$ is moreover easily found to verify homogeneous field equations and initial conditions in $\Omega_{\eta}^{\mathrm{E}}$.

Step 2. Energy of $\widetilde{\mathbf{e}}_{\eta}^{(k)}$. By Lemma 2.4, the elastodynamic energy $\mathcal{E}_{\eta}^{\mathrm{E}}\left(\widetilde{\mathbf{e}}_{\eta}^{(k)}, t\right)$ of the error field then verifies

$$
\frac{\mathrm{d}}{\mathrm{d} t} \mathcal{E}_{\eta}^{\mathrm{E}}\left(\widetilde{\mathbf{e}}_{\eta}^{(k)}, t\right)=-\int_{\Gamma}\left(\left[\mathbf{t}\left(\widetilde{\mathbf{e}}_{\eta}^{(k)}\right)\right]_{\eta} \cdot\left\langle\partial_{t} \widetilde{\mathbf{e}}_{\eta}^{(k)}\right\rangle_{\eta}+\left\langle\mathbf{t}\left(\widetilde{\mathbf{e}}_{\eta}^{(k)}\right)\right\rangle_{\eta} \cdot\left[\partial_{t} \widetilde{\mathbf{e}}_{\eta}^{(k)}\right]_{\eta}\right) \mathrm{d} \mathbf{y} .
$$

We now insert the values of $\left[\mathbf{t}\left(\widetilde{\mathbf{e}}_{\eta}^{(k)}\right)\right]_{\eta}$ and $\left\langle\mathbf{t}\left(\widetilde{\mathbf{e}}_{\eta}^{(k)}\right)\right\rangle_{\eta}$ given by (4.25) into (4.26). The homogeneous part of (4.25) coincides with the ETC (2.10), and for that reason gives rise to the same additional contribution to the energy (cf. (2.30)) as in the proof of Theorem 2.3. As a consequence, we obtain after time integration:

$$
\widetilde{\mathcal{E}}_{\eta}^{k}(t):=\mathcal{E}_{\eta}^{\mathrm{E}}\left(\widetilde{\mathbf{e}}_{\eta}^{(k)}, t\right)+\eta \mathcal{E}_{\eta}^{\mathrm{I}}\left(\widetilde{\mathbf{e}}_{\eta}^{(k)}, t\right)=-\eta^{k+1} \int_{0}^{t} \int_{\Gamma} \mathbf{F}_{\eta}^{k} \cdot\left\langle\partial_{t} \widetilde{\mathbf{e}}_{\eta}^{(k)}\right\rangle_{\eta} \mathrm{d} \mathbf{y} \mathrm{d} s+\eta^{k} \int_{0}^{t} \int_{\Gamma} \mathbf{G}_{\eta}^{k} \cdot\left[\partial_{t} \widetilde{\mathbf{e}}_{\eta}^{(k)}\right]_{\eta} \mathrm{d} \mathbf{y} \mathrm{d} s .
$$

having set $\mathbf{F}_{\eta}^{k}:=\left(\rho^{\mathrm{I}} \partial_{t}^{2}-\mathcal{P}_{2}\right)\left\langle\widetilde{\mathbf{u}}_{\eta}^{k}\right\rangle_{\eta}$ and $\mathbf{G}_{\eta}^{k}:=A\left[\widetilde{\mathbf{u}}_{\eta}^{k+1}\right]_{\eta}$ for convenience. After integration by parts for the second term in the right hand side, we get

$$
\widetilde{\mathcal{E}}_{\eta}^{k}(t)=-\eta^{k+1} \int_{0}^{t} \int_{\Gamma} \mathbf{F}_{\eta}^{k} \cdot\left\langle\partial_{t} \widetilde{\mathbf{e}}_{\eta}^{(k)}\right\rangle_{\eta} \mathrm{d} \mathbf{y} \mathrm{d} s-\eta^{k} \int_{0}^{t} \int_{\Gamma} \partial_{t} \mathbf{G}_{\eta}^{k} \cdot\left[\widetilde{\mathbf{e}}_{\eta}^{(k)}\right]_{\eta} \mathrm{d} \mathbf{y} \mathrm{d} s+\eta^{k} \int_{\Gamma} \mathbf{G}_{\eta}^{k}(\cdot, t) \cdot\left[\widetilde{\mathbf{e}}_{\eta}^{(k)}(\cdot, t)\right]_{\eta} \mathrm{d} \mathbf{y} \mathrm{d} s .
$$

Step 3. $O\left(\eta^{2 k}\right)$ estimate of $\widetilde{\mathcal{E}}_{\eta}^{k}(t)$. Contrary to step 3 for Lemma 4.4, we do not get directly a $O\left(\eta^{2 k+1}\right)$ estimate. Thanks to Lemma 4.2, and adopting again the notational conventions for the constants $C$ and $C_{k}(T)$ introduced in the proof of Lemma 4.4, we get

$$
\widetilde{\mathcal{E}}_{\eta}^{k}(t) \leqslant C_{k}(T)\left(\eta^{k+1} \int_{0}^{t}\left\|\left\langle\partial_{t} \widetilde{\mathbf{e}}_{\eta}^{(k)}\right\rangle_{\eta}\right\|_{L^{2}(\Gamma)} \mathrm{d} s+\eta^{k} \int_{0}^{t}\left\|\left[\widetilde{\mathbf{e}}_{\eta}^{(k)}\right]_{\eta}\right\|_{L^{2}(\Gamma)} \mathrm{d} s+\eta^{k}\left\|\left[\widetilde{\mathbf{e}}_{\eta}^{(k)}(\cdot, t)\right]_{\eta}\right\|_{L^{2}(\Gamma)}\right) .
$$

We now compare the terms in the right hand side to $\widetilde{\mathcal{E}}_{\eta}(t)$. From the definition $(2.30)$ of $\mathcal{E}_{\eta}^{\text {I }}$, we get:

$$
\frac{\eta \rho^{\mathrm{I}}}{2}\left\|\left\langle\partial_{t} \widetilde{\mathbf{e}}_{\eta}^{(k)}(\cdot, t)\right\rangle_{\eta}\right\|_{L^{2}(\Gamma)}^{2} \leqslant \eta \mathcal{E}_{\eta}^{\mathrm{I}}\left(\widetilde{\mathbf{e}}_{\eta}^{(k)}, t\right) \leqslant \widetilde{\mathcal{E}}_{\eta}^{k}(t) \Rightarrow\left\|\left\langle\partial_{t} \widetilde{\mathbf{e}}_{\eta}^{(k)}(\cdot, t)\right\rangle_{\eta}\right\|_{L^{2}(\Gamma)} \leqslant C \eta^{-1 / 2} \widetilde{\mathcal{E}}_{\eta}^{k}(t)^{1 / 2},
$$

while, using the trace theorem and Korn's inequality, we can claim that

$$
\left\|\left[\widetilde{\mathbf{e}}_{\eta}^{(k)}(\cdot, t)\right]_{\eta}\right\|_{L^{2}(\Gamma)}^{2} \leqslant C\left(\mathcal{E}_{\eta}^{\mathrm{E}}\left(\widetilde{\mathbf{e}}_{\eta}^{(k)}, t\right)^{1 / 2}+\int_{0}^{t} \mathcal{E}_{\eta}^{\mathrm{E}}\left(\widetilde{\mathbf{e}}_{\eta}^{(k)}, s\right)^{1 / 2} \mathrm{~d} s\right) \leqslant C\left(\widetilde{\mathcal{E}}_{\eta}^{k}(t)^{1 / 2}+\int_{0}^{t} \widetilde{\mathcal{E}}_{\eta}^{k}(s)^{1 / 2} \mathrm{~d} s\right) .
$$

Using (4.28) and (4.29) in (4.27) yields

$$
\widetilde{\mathcal{E}}_{\eta}^{k}(t) \leqslant C_{k}(T) \eta^{k}\left(\int_{0}^{t} \widetilde{\mathcal{E}}_{\eta}^{k}(s)^{1 / 2} \mathrm{~d} s+\widetilde{\mathcal{E}}_{\eta}^{k}(t)^{1 / 2}\right) .
$$

Then, proceeding as in the proof of Lemma 4.4 (Step 3), we conclude that

$$
\widetilde{\mathcal{E}}_{\eta}^{k}(t) \leqslant T C_{k}(T) \eta^{2 k}
$$

Step 4. $O\left(\eta^{2 k+2}\right)$ estimate of $\widetilde{\mathcal{E}}_{\eta}^{k}(t)$. This step is identical to step 4 of the proof of Lemma 4.4. 


\subsection{Proof of Theorem 2.5}

Proof of Theorem 2.5. The proof proceeds by recasting $\mathbf{u}_{\eta}^{\mathrm{E}}-\widetilde{\mathbf{u}}_{\eta}$ in the form

$$
\mathbf{u}_{\eta}^{\mathrm{E}}-\widetilde{\mathbf{u}}_{\eta}=\left(\mathbf{u}_{\eta}^{\mathrm{E}}-\mathbf{u}_{\eta}^{(2) \mathrm{E}}\right)+\left(\mathbf{u}_{\eta}^{(2) \mathrm{E}}-\widetilde{\mathbf{u}}_{\eta}^{(2)}\right)+\left(\widetilde{\mathbf{u}}_{\eta}^{(2)}-\widetilde{\mathbf{u}}_{\eta}\right) \quad \text { in } \Omega_{\eta}^{\mathrm{E}},
$$

where $\mathbf{u}_{\eta}^{(2) \mathrm{E}}$ and $\widetilde{\mathbf{u}}_{\eta}^{(2)}$ are the asymptotic approximations (4.1) and (4.20) with $k=2$. Each bracketed term is then estimated. The second term actually vanishes, since $\mathbf{u}_{\eta}^{(k) \mathrm{E}}=\widetilde{\mathbf{u}}_{\eta}^{(k)}$ for $k=2$, whereas estimates for the first and third terms are given by Lemmas 4.4 and 4.7 (with $k=2$ ), respectively. Theorem 2.5 then follows at once.

\section{Numerical approximation AND VALidation of the ETCs}

The aim of this section is to implement and demonstrate the ETCs (2.10). The semi-discretization in space is based on a finite element approximation of the elastodynamic problem in weak form. An energy analysis is used to establish the stability in time of this model. Several partially-implicit variants of an original explicit timestepping scheme are considered, with attention focused on estimating and comparing their respective critical time steps.

\subsection{Variational formulation and space discretization}

The spatial discretization is based on a weak formulation of problem (2.3)-(2.4a) and (2.10), for which a discrete equivalent of the energy conservation result of Theorem (2.3) will be shown to hold. The weak formulation is obtained by using the same approach as for obtaining the energy identity (2.31). The use of the second order ETC entails the introduction of a suitable functional space, namely:

$$
V=\left\{\mathbf{v} \in H^{1}\left(\Omega_{\eta}^{\mathrm{E}}\right)^{d} /\langle\mathbf{v}\rangle_{\eta} \in H^{1}(\Gamma)^{d}\right\}
$$

The resulting weak formulation reads:

$$
\frac{\mathrm{d}^{2}}{\mathrm{~d} t^{2}}\left(m\left(\widetilde{\mathbf{u}}_{\eta}, \mathbf{v}\right)+\eta m_{\Gamma}\left(\widetilde{\mathbf{u}}_{\eta}, \mathbf{v}\right)\right)+k\left(\widetilde{\mathbf{u}}_{\eta}, \mathbf{v}\right)+k_{\Gamma}^{\eta}\left(\widetilde{\mathbf{u}}_{\eta}, \mathbf{v}\right)=0, \quad \forall \mathbf{v} \in V .
$$

where we have introduced four continuous bilinear forms in $V$. The forms $m$ and $k$ involve volume integrals:

$$
m(\mathbf{u}, \mathbf{v}):=\int_{\Omega_{\eta}^{\mathrm{E}}} \rho \mathbf{u} \cdot \mathbf{v} \mathrm{d} \mathbf{x}, \quad k(\mathbf{u}, \mathbf{v}):=\int_{\Omega_{\eta}^{\mathrm{E}}} \boldsymbol{\sigma}(\mathbf{u}): \varepsilon(\mathbf{v}) \mathrm{d} \mathbf{x}
$$

and treat the two subdomains $\Omega_{\eta}^{ \pm}$as separated. By contrast, the forms $m_{\Gamma}$ and $k_{\Gamma}^{\eta}$, which involve surface integrals over $\Gamma$, couple the subdomains $\Omega_{\eta}^{ \pm}$(through the generalized jumps and averages):

$$
m_{\Gamma}(\mathbf{u}, \mathbf{v}):=\int_{\Gamma} \rho^{\mathrm{I}}\langle\mathbf{u}\rangle_{\eta} \cdot\langle\mathbf{v}\rangle_{\eta} \mathrm{d} \mathbf{y}, \quad k_{\Gamma}^{\eta}(\mathbf{u}, \mathbf{v}):=\eta^{-1} a_{\Gamma}(\mathbf{u}, \mathbf{v})+b_{\Gamma}(\mathbf{u}, \mathbf{v})+\eta p_{\Gamma}(\mathbf{u}, \mathbf{v})
$$

with the three bilinear forms $a_{\Gamma}(\cdot, \cdot), p_{\Gamma}(\cdot, \cdot)$ and $b_{\Gamma}(\cdot, \cdot)$ given by

$$
\begin{aligned}
a_{\Gamma}(\mathbf{u}, \mathbf{v}):= & \int_{\Gamma} A[\mathbf{u}]_{\eta} \cdot[\mathbf{v}]_{\eta} \mathrm{d} \mathbf{y} \\
p_{\Gamma}(\mathbf{u}, \mathbf{v}):= & \int_{\Gamma}\left(\left(\lambda^{\mathrm{I}}+\mu^{\mathrm{I}}\right) \operatorname{div}_{\Gamma}\langle\mathbf{u}\rangle_{\eta} \operatorname{div}_{\Gamma}\langle\mathbf{v}\rangle_{\eta}+\mu^{\mathrm{I}} \nabla_{\Gamma}\langle\mathbf{u}\rangle_{\eta}: \nabla_{\Gamma}\langle\mathbf{v}\rangle_{\eta}\right) \mathrm{d} \mathbf{y}, \\
b_{\Gamma}(\mathbf{u}, \mathbf{v}):= & \lambda^{\mathrm{I}} \int_{\Gamma}\left(\operatorname{div}_{\Gamma}\langle\mathbf{u}\rangle_{\eta}[\mathbf{v} \cdot \mathbf{n}]_{\eta}+\operatorname{div}_{\Gamma}\langle\mathbf{v}\rangle_{\eta}[\mathbf{u} \cdot \mathbf{n}]_{\eta}\right) \mathrm{d} \mathbf{y} \\
& +\mu^{\mathrm{I}} \int_{\Gamma}\left(\nabla_{\Gamma}\langle\mathbf{u} \cdot \mathbf{n}\rangle_{\eta} \cdot[\mathbf{v}]_{\eta}+\nabla_{\Gamma}\langle\mathbf{v} \cdot \mathbf{n}\rangle_{\eta} \cdot[\mathbf{u}]_{\eta}\right) \mathrm{d} \mathbf{y} .
\end{aligned}
$$


The reader will observe that $m(\cdot, \cdot), k(\cdot, \cdot), m_{\Gamma}(\cdot, \cdot), a_{\Gamma}(\cdot, \cdot), b_{\Gamma}(\cdot, \cdot)$, and $p_{\Gamma}(\cdot, \cdot)$ are symmetric and (with the exception of $\left.b_{\Gamma}(\cdot, \cdot)\right)$ positive. However, thanks to manipulatons similar to those made in the proof of Theorem 2.3, we also have that $k_{\Gamma}^{\eta}(\cdot, \cdot)$ is positive, since it is given (with $\varepsilon_{\eta, \Gamma}(\mathbf{u})$ given by $(2.27)$ ) by

$$
k_{\Gamma}^{\eta}(\mathbf{u}, \mathbf{u})=\int_{\Gamma}\left(\lambda^{\mathrm{I}} \operatorname{tr} \varepsilon_{\eta, \Gamma}(\mathbf{u}) \operatorname{tr} \varepsilon_{\eta, \Gamma}(\mathbf{u})+2 \mu^{\mathrm{I}} \varepsilon_{\eta, \Gamma}(\mathbf{u}): \varepsilon_{\eta, \Gamma}(\mathbf{u})\right) \mathrm{d} \mathbf{y} .
$$

Remark 5.1. Rigorously speaking, all bilinear forms depend on $\eta$. However, except for $k_{\Gamma}^{\eta}$, this dependence appears only through the integration domain $\Omega_{\eta}^{\mathrm{E}}$ or the generalized jumps and averages $[\cdot]_{\eta}$ and $\langle\cdot\rangle_{\eta}$, but this is not a real dependence since through a simple geometric transformation (translation), they coincide with bilinear forms that are independent of $\eta$. In particular, after finite element discretization in space, the associated matrices will depend only on the computational mesh. This is why we did not index them by $\eta$. In practice, if the overall computational domain is fixed, these matrices depend weakly on $\eta$ since the subdomain $\Omega_{\eta}^{\mathrm{E}}$ and its mesh do (through a slight stretching or compression along the through-thickness coordinate).

The finite element method is employed for the spatial discretization of (5.2) within a bounded region of interest (thereby setting an upper bound on the permissible analysis duration $T$ ). Let $V_{h}$ accordingly denote a subspace of $V$ of finite dimension $N_{h}$, where $h$ is an abstract approximation parameter: typically, any conforming Lagrange $\left(P_{k}\right.$ or $\left.Q_{k}\right)$ finite element space for $H^{1}\left(\Omega_{\eta}^{\mathrm{E}}\right)^{2}$ is suitable and $h$ denotes the mesh size of a (triangular or quadrangular) computational mesh $\mathcal{T}_{h}$ of $\Omega_{\eta}^{\mathrm{E}}$. Even though this is not mandatory, it is useful to require the facing meshes of $\partial \Omega_{\eta}^{+}$and $\partial \Omega_{\eta}^{-}$to have the same projection on the interface $\Gamma$ (see Fig. 2 for an illustration).

Denoting by $\mathbf{U}_{h}^{\eta} \in \mathbb{R}^{N_{h}}$ the vector gathering the coordinates of the semi-discrete approximation of $\widetilde{\mathbf{u}}_{\eta}$ in a chosen basis $\left\{\boldsymbol{\phi}_{i}\right\}$ of $V_{h}$ (in practice the standard Lagrange basis), a semi-discrete formulation of problem (5.2) can be expressed as a system of ODEs:

Find $\mathbf{U}_{h}$ in $V_{h}$ such that for all $t, 0 \leq t \leq T, \quad\left(\mathbf{M}_{h}+\eta \mathbf{M}_{h}^{\Gamma}\right) \frac{\mathrm{d}^{2}}{\mathrm{~d} t^{2}} \mathbf{U}_{h}^{\eta}(t)+\left(\mathbf{K}_{h}+\mathbf{K}_{h}^{\eta, \Gamma}\right) \mathbf{U}_{h}^{\eta}(t)=0$

where the matrices $\mathbf{M}_{h}$ and $\mathbf{K}_{h}$ are the standard (symmetric and positive) mass and stiffness matrices associated to the finite element approximation of the elastodynamics equations in $\Omega_{\eta}^{\mathrm{E}}$ :

$$
\left(\mathbf{M}_{h}\right)_{i j}=\oint_{\Omega_{\eta}^{\mathrm{E}}} \rho \phi_{i} \cdot \phi_{j} \mathrm{~d} \mathbf{x}, \quad\left(\mathbf{K}_{h}\right)_{i j}=\oint_{\Omega_{\eta}^{\mathrm{E}}} \boldsymbol{\sigma}\left(\phi_{i}\right): \varepsilon\left(\phi_{j}\right) \mathrm{d} \mathbf{x}
$$

$\mathbf{M}_{h}^{\Gamma}$ is a symmetric and positive matrix given by

$$
\left(\mathbf{M}_{h}^{\Gamma}\right)_{i j}=\oint_{\Gamma} \rho^{\mathrm{I}}\left\langle\phi_{i}\right\rangle_{\eta} \cdot\left\langle\phi_{j}\right\rangle_{\eta} \mathrm{d} \mathbf{y}
$$

while the "boundary stiffness matrix" (whose only nonzero entries are associated to degrees of freedom located on $\partial \Omega_{\eta}^{+}$or $\partial \Omega_{\eta}^{-}$) is also symmetric and positive thanks to (5.5) and can be decomposed as the sum of three symmetric matrices:

$$
\mathbf{K}_{h}^{\eta, \Gamma}=\eta^{-1} \mathbf{A}_{h}+\left(\mathbf{B}_{h}+\mathbf{B}_{h}^{T}\right)+\eta \mathbf{P}_{h}
$$

where respectively

$$
\begin{aligned}
\left(\mathbf{P}_{h}\right)_{i j} & =\oint_{\Gamma}\left(\left(\lambda^{\mathrm{I}}+\mu^{\mathrm{I}}\right) \operatorname{div}_{\Gamma}\left\langle\boldsymbol{\phi}_{i}\right\rangle_{\eta} \operatorname{div}_{\Gamma}\left\langle\boldsymbol{\phi}_{j}\right\rangle_{\eta}+\mu^{\mathrm{I}} \nabla_{\Gamma}\left\langle\boldsymbol{\phi}_{i}\right\rangle_{\eta}: \nabla_{\Gamma}\left\langle\boldsymbol{\phi}_{j}\right\rangle_{\eta}\right) \mathrm{d} \mathbf{y}, \\
\left(\mathbf{A}_{h}\right)_{i j} & =\oint_{\Gamma} A\left[\boldsymbol{\phi}_{i}\right] \cdot\left[\boldsymbol{\phi}_{j}\right] \mathrm{d} \mathbf{y}, \quad\left(\mathbf{B}_{h}\right)_{i j}=\lambda^{\mathrm{I}} \oint_{\Gamma} \operatorname{div}_{\Gamma}\left\langle\boldsymbol{\phi}_{i}\right\rangle_{\eta}\left[\boldsymbol{\phi}_{j} \cdot \mathbf{n}\right]_{\eta} \mathrm{d} \mathbf{y}+\mu^{\mathrm{I}} \oint_{\Gamma} \nabla_{\Gamma}\left\langle\boldsymbol{\phi}_{i} \cdot \mathbf{n}\right\rangle_{\eta}\left[\boldsymbol{\phi}_{j}\right]_{\eta} \mathrm{d} \mathbf{y} .
\end{aligned}
$$



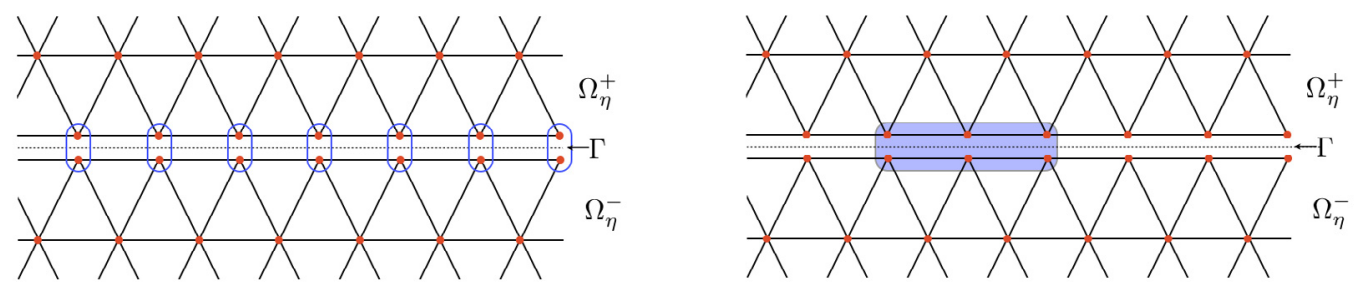

Figure 2. Connectivity of the matrix $\mathbf{M}_{h}^{\Gamma}$ and $\mathbf{A}_{h}$ (left), $\mathbf{B}_{h}$ and $\mathbf{P}_{h}$ (right) with $P_{1}$ finite elements.

In (5.7) and (5.9), the symbol $\oint$ refers to the fact that the integrals are evaluated (approximately) through quadrature formulas with positive quadrature weights, which are essential to ensure the announced positivity properties of the matrices and guarantee in the same way the stability of the semi-discrete problem through the conservation of the semi-discrete energy $\mathcal{E}_{h}^{\eta}$ defined by

$$
\mathcal{E}_{h}^{\eta}=\frac{1}{2}\left(\frac{\mathrm{d}}{\mathrm{d} t} \mathbf{U}_{h}^{\eta}\right)^{T}\left(\mathbf{M}_{h}+\eta \mathbf{M}_{h}^{\Gamma}\right) \frac{\mathrm{d}}{\mathrm{d} t} \mathbf{U}_{h}^{\eta}+\frac{1}{2}\left(\mathbf{U}_{h}^{\eta}\right)^{T}\left(\mathbf{K}_{h}+\mathbf{K}_{h}^{\eta, \Gamma}\right) \mathbf{U}_{h}^{\eta}
$$

In this work, we choose to focus on the use of higher-order spectral finite elements [6], whose geometrical degrees of freedom (Lagrange interpolation points) are chosen to coincide with the (Gauss-Lobatto) quadrature nodes, because this is the situation where ETCs are expected to be the most useful. All integrals over $\Gamma$ are discretized with 2D versions of spectral elements, so that mass lumping is achieved for $\mathbf{M}_{h}^{\Gamma}$ and $\mathbf{A}_{h}$, in addition to $\mathbf{M}_{h}$ [6]. Indeed, such finite element methods can achieve satisfactory accuracy with relatively large elements, so that situations where $h$ is significantly larger than $\eta$ may arise. It is then important to avoid the geometrical constraint induced by the length scale $\eta$. Moreover, spectral elements provide mass lumping and, importantly for our present purposes, particular connectivity properties for the boundary matrices. More precisely:

(i) The matrices $\mathbf{M}_{h}^{\Gamma}$ and $\mathbf{A}_{h}$ are block-diagonal with $2 d \times 2 d$ blocks (remember that two unknowns - one per displacement component - are attached to each geometrical node) since they only connect pairs of nodes on $\partial \Omega_{\eta}^{+}$and $\partial \Omega_{\eta}^{-}$that have the same projection on $\Gamma$ (Fig. 2 left). In fact, closer examination shows that (after renumbering the local degrees of freedom) they are even block-diagonal with $2 \times 2$ blocks. As a consequence, their inversion is explicit.

(ii) The matrices $\mathbf{B}_{h}$ and $\mathbf{P}_{h}$ connect all unknowns associated to neighbouring boundary nodes, i.e. nodes that either belong to the support of the same basis function, or have the same projection on $\Gamma$ (Fig. 2 right). As a consequence their inversion is implicit.

These connectivity properties are illustrated by Figure 2 in the case of $P_{1}$ finite elements.

\subsection{Time-domain approximation}

We investigate in this section several time stepping schemes whose common property is to lead to an explicit scheme for updating the unknowns associated to all nodes not located on one of the interfaces $\partial \Omega_{\eta}^{ \pm}$. To this end, we shall study schemes which are "interface modifications" of the standard leap-frog scheme used in the case where all boundary matrices are ignored. The latter corresponds to solving two uncoupled free boundary problems (one for each subdomain $\partial \Omega_{\eta}^{ \pm}$), yielding the semi-discrete problem:

$$
\mathbf{M}_{h} \frac{\mathrm{d}^{2}}{\mathrm{~d} t^{2}} \mathbf{U}_{h}(t)+\mathbf{K}_{h} \mathbf{U}_{h}(t)=0
$$

Let $\Delta t>0$ be a given time step and set $t^{n}=n \Delta t, \mathbf{U}_{h}^{n} \simeq \mathbf{U}_{h}\left(t^{n}\right)(n \in \mathbb{N})$. The corresponding fully discrete scheme (which is truly explicit thanks to mass lumping), thereafter referred to as "Scheme 0", is then

$$
\mathbf{M}_{h} \frac{\mathbf{U}_{h}^{n+1}-2 \mathbf{U}_{h}^{n}+\mathbf{U}_{h}^{n-1}}{\Delta t^{2}}+\mathbf{K}_{h} \mathbf{U}_{h}^{n}=0
$$




\subsubsection{Definition of three time stepping schemes}

Scheme 1 (fully explicit): this is scheme 0, i.e. (5.10), with the relevant layer contributions added to $\mathbf{M}_{h}$ and $\mathbf{K}_{h}$ :

$$
\left(\mathbf{M}_{h}+\eta \mathbf{M}_{h}^{\Gamma}\right) \frac{\left(\mathbf{U}_{h}^{\eta}\right)^{n+1}-2\left(\mathbf{U}_{h}^{\eta}\right)^{n}+\left(\mathbf{U}_{h}^{\eta}\right)^{n-1}}{\Delta t^{2}}+\left(\mathbf{K}_{h}+\mathbf{K}_{h}^{\eta, \Gamma}\right)\left(\mathbf{U}_{h}^{\eta}\right)^{n}=0
$$

Scheme 2 (quasi-explicit):

$$
\begin{aligned}
\left(\mathbf{M}_{h}+\eta \mathbf{M}_{h}^{\Gamma}\right) \frac{\left(\mathbf{U}_{h}^{\eta}\right)^{n+1}-2\left(\mathbf{U}_{h}^{\eta}\right)^{n}+\left(\mathbf{U}_{h}^{\eta}\right)^{n-1}}{\Delta t^{2}}+\mathbf{K}_{h}\left(\mathbf{U}_{h}^{\eta}\right)^{n} & \\
& +\eta^{-1} \mathbf{A}_{h} \frac{\left(\mathbf{U}_{h}^{\eta}\right)^{n+1}+2\left(\mathbf{U}_{h}^{\eta}\right)^{n}+\left(\mathbf{U}_{h}^{\eta}\right)^{n-1}}{4}+\left(\mathbf{B}_{h}+\mathbf{B}_{h}^{T}\right)\left(\mathbf{U}_{h}^{\eta}\right)^{n}+\eta \mathbf{P}_{h}\left(\mathbf{U}_{h}^{\eta}\right)^{n}=0 .
\end{aligned}
$$

In this scheme, only the term $\mathbf{A}_{h} \mathbf{U}_{h}$ is treated in an implicit way. However, solving (5.12) for $\left(\mathbf{U}_{h}^{\eta}\right)^{n+1}$ remains explicit in practice because of the block-diagonal structure of $\mathbf{A}_{h}$ (see item (i) in Sect. 5.1).

Scheme 3 (semi-implicit):

$$
\left(\mathbf{M}_{h}+\eta \mathbf{M}_{h}^{\Gamma}\right) \frac{\left(\mathbf{U}_{h}^{\eta}\right)^{n+1}-2\left(\mathbf{U}_{h}^{\eta}\right)^{n}+\left(\mathbf{U}_{h}^{\eta}\right)^{n-1}}{\Delta t^{2}}+\mathbf{K}_{h}\left(\mathbf{U}_{h}^{\eta}\right)^{n}+\mathbf{K}_{h}^{\eta, \Gamma} \frac{\left(\mathbf{U}_{h}^{\eta}\right)^{n+1}+2\left(\mathbf{U}_{h}^{\eta}\right)^{n}+\left(\mathbf{U}_{h}^{\eta}\right)^{n-1}}{4}=0 .
$$

The whole term $\mathbf{K}_{h}^{\eta, \Gamma} \mathbf{U}_{h}$ is treated implicitly. This scheme is truly implicit on the interface since the updating of the interface unknowns requires the resolution of a (banded) linear system (see item (i) in Sect. 5.1). For this reason, if the same time step $\Delta t$ is chosen, scheme 3 is moderately more expensive than schemes 1 or 2 (assuming the number of interfacial DOFs to be a small fraction of the total number of DOFs).

\subsubsection{A comparative stability analysis of the three schemes.}

Our main concern in the forthcoming discussion is the robustness of our numerical scheme with respect to the small parameter $\eta$. More precisely, we aim at determining whether the time step limit arising from the CFL stability condition of the scheme is sensitive to $\eta$ in the limit $\eta \rightarrow 0$. Depending on the outcome (yes or no), the scheme shall be deemed robust or not robust.

Theoretical stability analysis. Since what follows is quite classical, some details will be omitted. We start from the well-known result stating that any numerical scheme of the form:

$$
\mathbf{M}_{h}^{\star} \frac{\mathbf{U}_{h}^{n+1}-2 \mathbf{U}_{h}^{n}+\mathbf{U}_{h}^{n-1}}{\Delta t^{2}}+\mathbf{K}_{h}^{\star} \frac{\mathbf{U}_{h}^{n+1}+2 \mathbf{U}_{h}^{n}+\mathbf{U}_{h}^{n-1}}{4}=0,
$$

is stable as soon as $\mathbf{M}_{h}^{\star}$ and $\mathbf{K}_{h}^{\star}$ are positive. To be easily convinced, it suffices to refer to the conservation of the discrete energy

$$
\left(\mathcal{E}_{h}^{\star}\right)^{n+1 / 2}:=\frac{1}{2}\left(\frac{\mathbf{U}_{h}^{n+1}-\mathbf{U}_{h}^{n}}{\Delta t}\right) \mathbf{M}_{h}^{\star}\left(\frac{\mathbf{U}_{h}^{n+1}-\mathbf{U}_{h}^{n}}{\Delta t}\right)+\frac{1}{2}\left(\frac{\mathbf{U}_{h}^{n+1}+\mathbf{U}_{h}^{n}}{2}\right) \mathbf{K}_{h}^{\star}\left(\frac{\mathbf{U}_{h}^{n+1}+\mathbf{U}_{h}^{n}}{2}\right) .
$$

Hence, if the positivity of $\mathbf{K}_{h}^{\star}$ is known beforehand, the positivity of $\mathbf{M}_{h}^{\star}$ is a necessary and sufficient stability condition. Indeed, if not, there exists a strictly negative eigenvalue $\lambda$ for the generalized eigenvalue problem

$$
\mathbf{K}_{h}^{\star} \mathbf{U}_{h}=\lambda \mathbf{M}_{h}^{\star} \mathbf{U}_{h}, \quad \mathbf{U}_{h} \neq 0 .
$$

Next, we observe that each of our schemes can be put in the form (5.14) by choosing $\mathbf{M}_{h}^{\star}$ and $\mathbf{K}_{h}^{\star}$ as follows:
(a) Scheme 0: $\quad \mathbf{M}_{h}^{\star}=\mathbf{M}_{h}-\frac{1}{4} \Delta t^{2} \mathbf{K}_{h}$,
(b) Scheme 1: $\quad \mathbf{M}_{h}^{\star}=\mathbf{M}_{h}+\eta \mathbf{M}_{h}^{\Gamma}-\frac{1}{4} \Delta t^{2}\left(\mathbf{K}_{h}+\mathbf{K}_{h}^{\eta, \Gamma}\right)$,
$\mathbf{K}_{h}^{\star}=\mathbf{K}_{h}$
(c) Scheme 2: $\quad \mathbf{M}_{h}^{\star}=\mathbf{M}_{h}+\eta \mathbf{M}_{h}^{\Gamma}-\frac{1}{4} \Delta t^{2}\left(\mathbf{K}_{h}+\mathbf{B}_{h}+\mathbf{B}_{h}^{T}+\eta \mathbf{P}_{h}\right)$,
$\mathbf{K}_{h}^{\star}=\mathbf{K}_{h}+\mathbf{K}_{h}^{\eta, \Gamma}$
(d) Scheme 3: $\mathbf{M}_{h}^{\star}=\mathbf{M}_{h}+\eta \mathbf{M}_{h}^{\Gamma}-\frac{1}{4} \Delta t^{2} \mathbf{K}_{h}$,
$\mathbf{K}_{h}^{\star}=\mathbf{K}_{h}+\mathbf{K}_{h}^{\eta, \Gamma}$
$\mathbf{K}_{h}^{\star}=\mathbf{K}_{h}+\mathbf{K}_{h}^{\eta, \Gamma}$ 
In all cases, due to the positivity of both $\mathbf{K}_{h}^{\eta, \Gamma}$ and $\mathbf{K}_{h}$, the matrix $\mathbf{K}_{h}^{\star}$ is positive. Thus, demanding that the matrix $\mathbf{M}_{h}^{\star}$ be positive provides the sought stability condition.

- For the (reference) scheme 0, the stability condition reads $\mathbf{M}_{h}-4 \Delta t^{2} \mathbf{K}_{h} \geq 0$, implying the well-known CFL condition

$$
\Delta t \leq \Delta t_{0}^{c}(h):=2 \operatorname{sr}\left(\mathbf{M}_{h}^{-1} \mathbf{K}_{h}\right)^{-1 / 2}
$$

where $\operatorname{sr}(\cdot)$ refers to the spectral radius of a matrix and the superscript $c$ stands for "critical".

- For the (semi-implicit) scheme 3, the stability condition becomes

$$
\Delta t \leq \Delta t_{3}^{c}(\eta, h):=2 \operatorname{sr}\left(\left[\mathbf{M}_{h}+\eta \mathbf{M}_{h}^{\Gamma}\right]^{-1} \mathbf{K}_{h}\right)^{-1 / 2}
$$

which is strictly less severe than (5.16), since $\mathbf{M}_{h}^{\Gamma}$ is positive. In particular (5.16) is a uniform (in $\eta$ ) sufficient stability condition for scheme 3 . Moreover, for small $\eta(5.17)$ reads

$$
\Delta t_{3}^{c}(\eta, h)=\Delta t_{0}^{c}(h)(1+O(\eta)) \quad(\text { with } O(\eta) \geq 0) .
$$

This establishes the robustness with respect to $\eta$ of the scheme 3 .

- By contrast, for the (fully explicit) scheme 1, the stability condition becomes

$$
\Delta t \leq \Delta t_{1}^{c}(\eta, h):=2 \operatorname{sr}\left(\left[\mathbf{M}_{h}+\eta \mathbf{M}_{h}^{\Gamma}\right]^{-1}\left[\mathbf{K}_{h}+\mathbf{K}_{h}^{\eta, \Gamma}\right]\right)^{-1 / 2} .
$$

It is then easy to see that the scheme is not robust. Indeed,

$$
\lim _{\eta \rightarrow 0} \eta\left(\mathbf{M}_{h}+\eta \mathbf{M}_{h}^{\Gamma}\right)^{-1}\left(\mathbf{K}_{h}+\mathbf{K}_{h}^{\eta, \Gamma}\right)=\mathbf{M}_{h}^{-1} \mathbf{A}_{h}
$$

means that

$$
\Delta t_{1}^{c}(\eta, h) \leq 2 \eta^{1 / 2} \operatorname{sr}\left(\mathbf{M}_{h}^{-1} \mathbf{A}_{h}\right)^{-1 / 2}(1+O(\eta))
$$

- The properties of scheme 2 are in-between those of schemes 1 and 3 . The stability condition is

$$
\Delta t \leq \Delta t_{2}^{c}(\eta, h):=2 \operatorname{sr}\left(\left[\mathbf{M}_{h}+\eta \mathbf{M}_{h}^{\Gamma}\right]^{-1}\left[\mathbf{K}_{h}+\mathbf{B}_{h}+\mathbf{B}_{h}^{T}+\eta \mathbf{P}_{h}\right]\right)^{-1 / 2} .
$$

The scheme 2 is robust since (5.21) reads

$$
\Delta t_{2}^{c}(\eta, h)=2 \operatorname{sr}\left(\mathbf{M}_{h}^{-1}\left[\mathbf{K}_{h}+\mathbf{B}_{h}+\mathbf{B}_{h}^{T}\right]\right)^{-1 / 2}(1+O(\eta)) .
$$

The symmetric matrix $\mathbf{B}_{h}+\mathbf{B}_{h}^{T}$ having no sign, it is difficult to compare easily $\Delta t_{2}^{c}(\eta, h)$ and $\Delta t_{3}^{c}(\eta, h)$. However, since the corresponding quadratic form can take positive values, one can conjecture that $\Delta t_{2}^{c}(\eta, h)<$ $\Delta t_{3}^{c}(\eta, h)$, i.e. that condition (5.21) is more severe than (5.17); this will be corroborated by our numerical results.

Remark 5.2. In the particular case where (i) the coefficients $(\rho, \lambda, \mu)$ are constant (homogeneous reference medium), (ii) a uniform mesh (made of rectangles or bricks, depending on the space dimension) is used and (iii) $Q_{k}$ finite elements are considered, it is easy to describe the asymptotic behaviour of the CFL limits $\Delta t_{j}^{c}(\eta, h)$ for small $\eta$ and $h$. Let $\nu:=\lambda /[2(\lambda+\mu)]$ and $V_{P}:=((\lambda+2 \mu) / \rho)^{1 / 2}$ respectively denote Poisson's ratio and the $\mathrm{P}$-wave velocity in the reference medium, and $\nu^{\mathrm{I}}, V_{P}^{\mathrm{I}}$ the corresponding quantities for the layer medium. Then:

(a) For schemes 0 and 3 , there exists a (dimensionless) constant $C_{j, k}(v)$ such that:

$$
\Delta t_{0}^{c}(h)=C_{k}(\nu)\left(h / V_{P}\right)(1+O(h)), \quad \Delta t_{3}^{c}(\eta, h)=C_{k}(\nu)\left(h / V_{P}\right)(1+O(h+\eta)) .
$$

(b) For the scheme 1, there exists a (dimensonless) constant $C_{1, k}\left(\nu^{\mathrm{I}}\right)$ such that:

$$
\Delta t_{1}^{c}(\eta, h)=C_{1, k}\left(\nu^{\mathrm{I}}\right)\left(\sqrt{h \eta} / V_{P}^{\mathrm{I}}\right)(1+O(h+\eta)) .
$$

(c) Finally, for the scheme 2, there exists a (dimensonless) constant $C_{2, k}\left(\nu, \nu^{\mathrm{I}}, V_{P}^{\mathrm{I}} / V_{P}\right)$ such that:

$$
\Delta t_{2}^{c}(\eta, h)=C_{2, k}\left(\nu, \nu^{\mathrm{I}}, V_{P}^{\mathrm{I}} / V_{P}\right)\left(h / V_{P}\right)(1+O(h+\eta)) .
$$




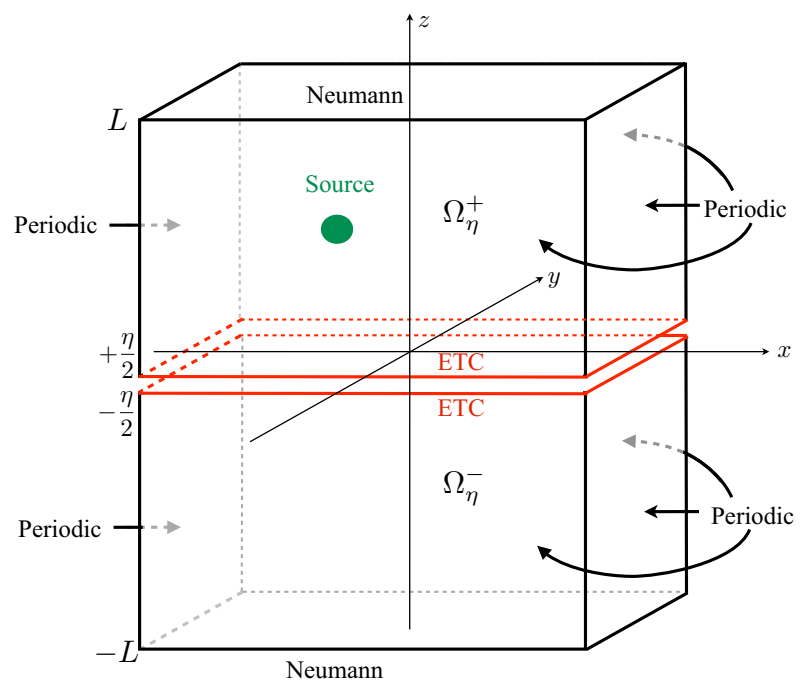

Figure 3. Configuration for the computational experiments (3D case).

\subsection{Setup for numerical experiments}

Numerical experiments were conducted for a bounded domain $\Omega=]-L, L[$, with the layer region defined by $\left.\left.\Omega_{\eta}^{\mathrm{I}}:=\right]-L, L{ }^{d-1} \times\right]-\eta / 2, \eta / 2\left[\right.$ and $\Omega_{\eta}^{\mathrm{E}}=\Omega \backslash \overline{\Omega_{\eta}^{\mathrm{I}}}$ (see Fig. 3). Homogeneous Neumann boundary conditions are assumed on the top and bottom surfaces $]-L, L{ }^{d-1} \times\{ \pm L\}$, the remaining parts of $\partial \Omega$ supporting periodic conditions. Regular meshes made of quadrilateral $(d=2)$ or hexahedral $(d=3)$ elements are used, with Lagrange interpolation, Gauss-Lobatto interpolation points and Gauss-Lobatto quadrature formulas.

Unless stated otherwise, the excitation consists of a body force distribution $\mathbf{g}$ along the $\mathbf{e}_{1}$ direction, having a truncated Gaussian spatial variation and with a Ricker pulse time modulation, i.e. of the form

$$
\begin{aligned}
\mathbf{g}(\mathbf{x}, t) & =\exp \left(-\alpha\left\|\mathbf{x}-\mathbf{x}_{0}\right\|^{2}\right) \frac{\mathrm{d}^{2}}{\mathrm{~d} t^{2}} \exp \left(-\pi^{2}\left(f_{0} t-1\right)^{2}\right) \mathbf{e}_{1} & & \left\|\mathbf{x}-\mathbf{x}_{0}\right\| \leq 1 \\
& =0 & & \left\|\mathbf{x}-\mathbf{x}_{0}\right\|>1
\end{aligned}
$$

where $\mathbf{x}_{0}$ is the source center (chosen so that the source support does not intersect the layer), $f_{0}$ the central frequency, and with $\alpha=6 \ln 10$ (so that $|\mathbf{g}(\cdot, t)| /|\mathbf{g}(0, t)|=10^{-6}$ along the edge of the truncated Gaussian)

The computational domain $\Omega_{\eta}^{\mathrm{E}}$ is meshed by starting from a regular mesh of $\Omega$ with nodes located on $\Gamma$. The latter are then duplicated and translated by vectors $\pm \frac{\eta}{2} \mathbf{n}$. Figure 4 shows meshes made using this procedure.

\subsection{Comparative study of CFL conditions}

For the case of a two-dimensional domain $(d=2)$ with $L=5$, the critical time steps $\Delta t^{c}(\eta, h)$, respectively defined by (5.17), (5.19) or (5.21)) for scheme 1, 2 or 3, are computed as functions of $\eta$ and compared to the reference value $\Delta t_{0}^{c}(h)$ given by (5.16). The material parameters are set to $\left(\rho^{-}, \mu^{-}, \lambda^{-}\right)=\left(\rho^{+}, \mu^{+}, \lambda^{+}\right)=(1,1,1)$ outside the layer, and $\left(\rho^{\mathrm{I}}, \lambda^{\mathrm{I}}, \mu^{\mathrm{I}}\right)=(2,10,6)$ inside the layer. The spatial discretization uses quadrilateral finite element of order 10 and size $\Delta x=\Delta z=1$ (see Fig. 4).

Figure 5a shows the relative CFL limit $\Delta t_{j}^{c}(\eta, h) / \Delta t_{0}^{c}(h)$ as a function of $\eta$ for each scheme (the values of $\eta$ have to be compared typically with $h=1$ ). The numerical results reproduce the theoretical expectations of Section 5.2. The CFL of the fully-explicit scheme (5.11) sharply deteriorates in the thin-layer limit, making it unsuitable, as expected (the slope of the leftmost part of the corresponding curve in log-log scale is in fact found to be numerically close to $1 / 2$, consistently with the predicted asymptotic behavior $(5.20)$ of $\left.\Delta t_{1}^{c}(\eta, h)\right)$. 


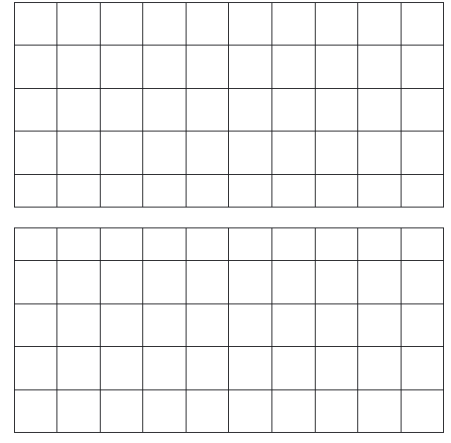

(a) 2D mesh (used in Sect. 5.4)

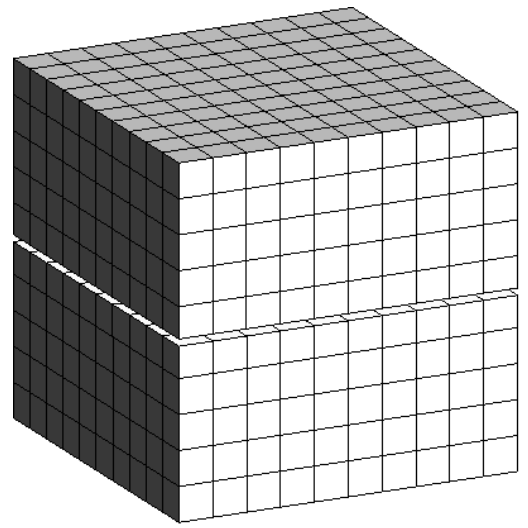

(b) 3D mesh (used in Sect. 5.5.2)

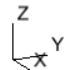

Figure 4. 2D and 3D meshes.

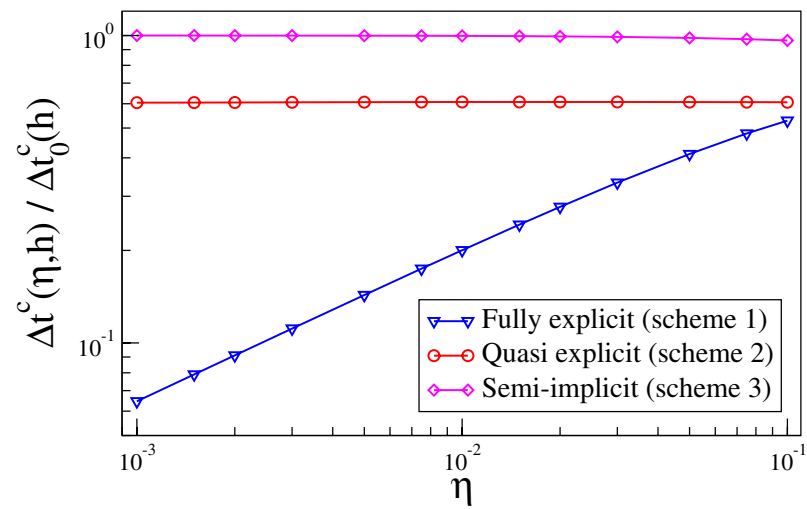

(a) as a function of $\eta$

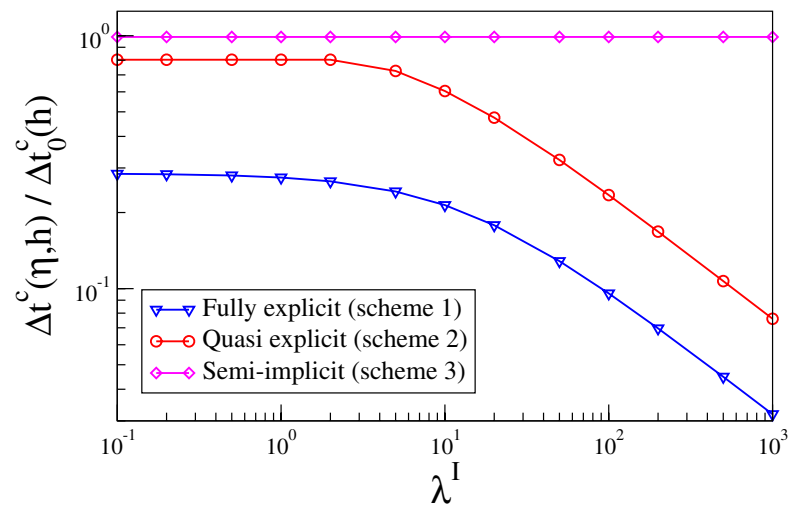

(b) as a function of $\lambda^{\mathrm{I}}$, with $\eta=0.01$

Figure 5. Critical time step $\Delta t^{c}(\eta, h)$ for schemes 1,2 and 3.

By contrast, the CFL of the semi-implicit scheme (5.13) is only weakly dependent on $\eta$ and converges to the reference CFL $\Delta t_{0}^{c}$ in the thin-layer limit. Finally, the CFL of the quasi-explicit scheme (5.12), while independent of $\eta$, has a lower value $\Delta t_{2}^{c} \approx 0.6 \Delta t_{0}^{c}$ which makes it somewhat less attractive than (5.13).

It is also interesting to evaluate the influence of the material parameters inside the layer on the stability condition. Figure 5b shows for each scheme the dependence of the critical time step on the Lamé modulus $\lambda^{\mathrm{I}}$ (which only influences the boundary matrices $\mathbf{A}_{h}, \mathbf{B}_{h}$ and $\mathbf{P}_{h}$ ), for $\eta=0.01$ and $\rho^{\mathrm{I}}, \mu^{\mathrm{I}}$ kept fixed. Only the semi-implicit scheme (5.13) appears to be essentially independent of the layer material. This is fully consistent with theoretical expectations, as equations (5.17), (5.19) and (5.21) predict the stability condition of scheme 3 to be insensitive to $\lambda^{\mathrm{I}}$, but that of scheme 1 or 2 to deteriorate for large values of $\lambda^{\mathrm{I}}$ (i.e. very stiff layers).

To conclude, the (semi-implicit) scheme 3 is best, by virtue of being fairly insensitive to either the thickness or the material parameters of the layer and having a CFL limit $\Delta t^{c}$ close in all cases to the reference value $\Delta t_{0}^{c}$, despite the fact that it requires the solution of a (small and sparse) linear system for updating the interface unknowns. The quasi-explicit scheme is a reasonable compromise, since it is robust in $\eta$, provided that the material contrast is not too large (otherwise, the gain brought by avoiding the solution of a linear system does 

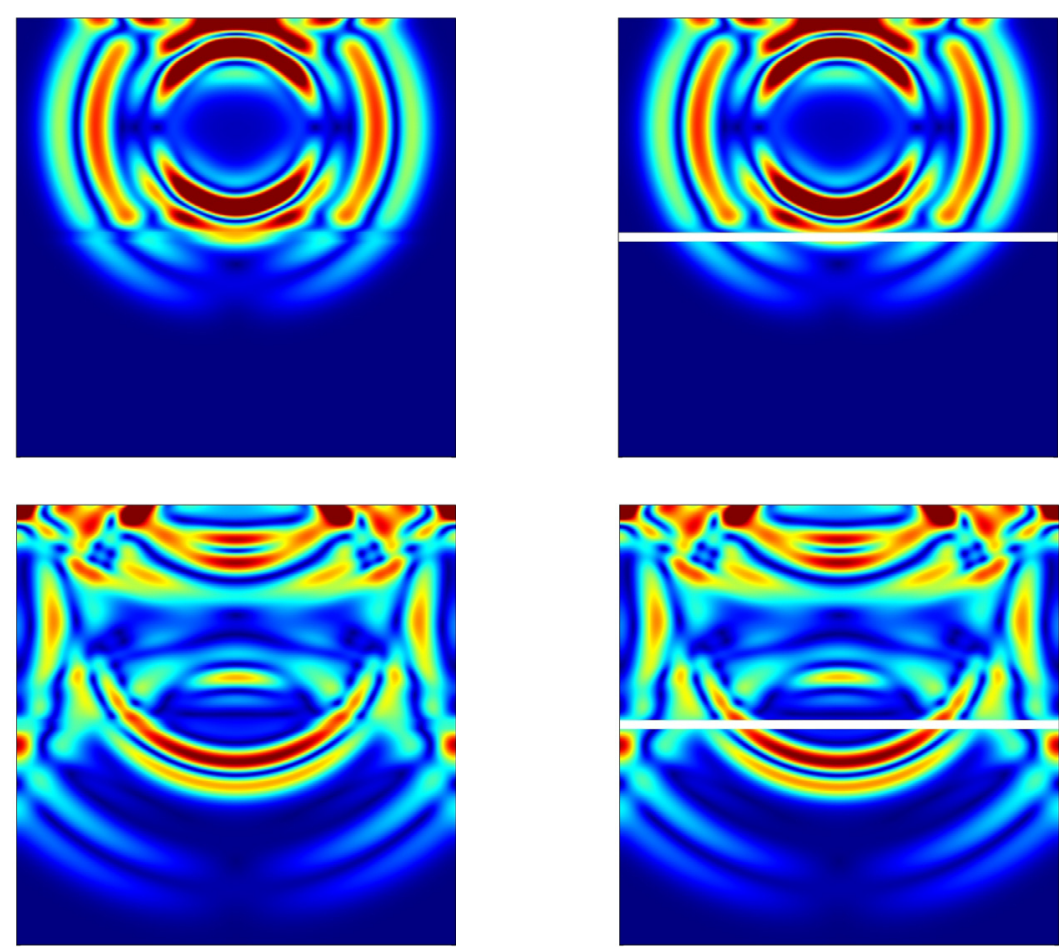

FiguRE 6. 2-D case: displacement moduli of the exact (left) and effective (right) transmission solutions (with $\eta=0.2$ ), evaluated at $t=3($ top $)$ and $t=4.5$ (bottom).

not compensate the reduction of the time step). Finally, the fully explicit scheme is unsuitable because its stability condition is not robust with respect to variations of the layer thickness and material parameters.

\subsection{A numerical validation of the ETCs}

\subsubsection{2-D numerical experiments}

The computational domain is again such that $L=H=5$. The material parameters are chosen as $\left(\rho^{-}, \lambda^{-}, \mu^{-}\right)=\left(\rho^{+}, \lambda^{+}, \mu^{+}\right)=(1,1,1)$ outside the layer, and $\left(\rho^{\mathrm{I}}, \lambda^{\mathrm{I}}, \mu^{\mathrm{I}}\right)=(2,4,3)$ inside the layer. The source is of the form (5.23), with $\mathbf{x}_{0}=(0,2.5)$ and $f_{0}=0.98$. The spatial discretization uses quadrilateral finite element of order 10 and size $\Delta x=\Delta z=1 / 3$. The time-stepping scheme (5.13) is used, with a time step $\Delta t=5 \times 10^{-5}$. The solution $\mathbf{u}_{\eta}$ computed using this discretization is very close (within a relative error smaller than $10^{-6}$ ) to a reference solution computed using a highly refined space and time discretization. The effective transmission solution for $\eta=0.2$ is shown for illustration purposes on Figure 6 , for times $t=3.5$ and $t=5$.

We now compare the computed exact and effective transmission solutions, by evaluating for a range of values of $\eta$ the relative difference $E(\eta)$ in $L^{2}$ norm between the computed approximations of $\mathbf{u}_{\eta}$ and $\widetilde{\mathbf{u}}_{\eta}$, defined by

$$
E(\eta ; t):=\left\|\mathbf{u}_{h}^{\eta}(\cdot, t)-\tilde{\mathbf{u}}_{h}^{\eta}(\cdot, t)\right\|_{L^{2}\left(\Omega_{\eta}^{\mathrm{E}}\right)} /\left\|\mathbf{u}_{h}^{\eta}(\cdot, t)\right\|_{L^{2}\left(\Omega_{\eta}^{\mathrm{E}}\right)} .
$$

Figure 7 shows that $E(\eta ; 5)=O\left(\eta^{3}\right)$, hence giving a numerical validation of Theorem 2.5, whereas the solution $\check{\mathbf{u}}_{\eta}$ for the order 0 ETC, which just assumes $\left\{\check{\mathbf{u}}_{\eta}\right\}_{\eta}=0$, is seen to yield a $O(\eta)$ error, also as expected. 


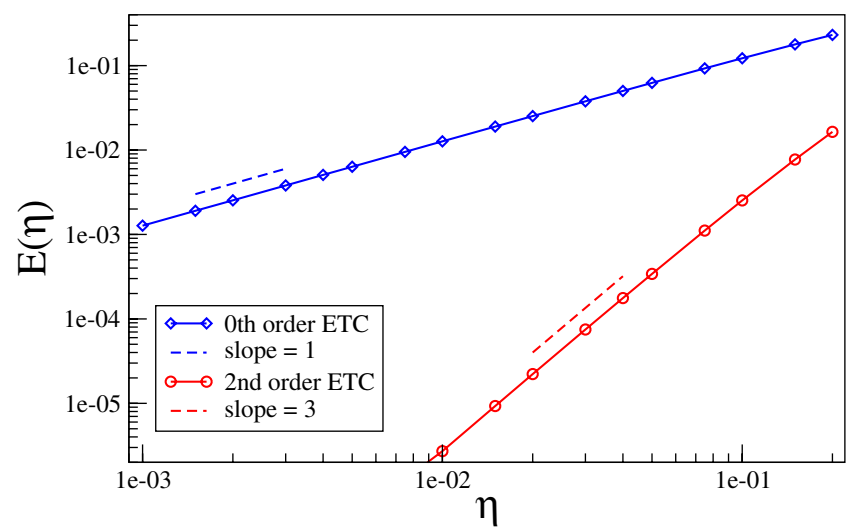

FiguRE 7. 2-D case: relative $L^{2}$ difference $E(\eta ; 5)$ as a function of $\eta$ for (i) the second-order ETC model and (ii) the model where the layer is ignored.

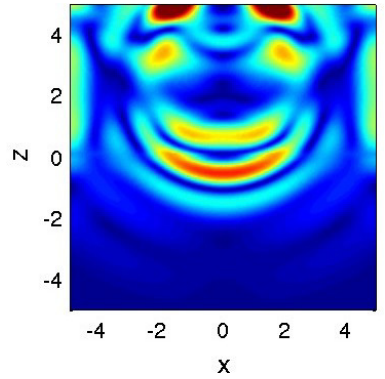

(a) $\left|\mathbf{u}_{\eta}(x, 0, z, 5)\right|$

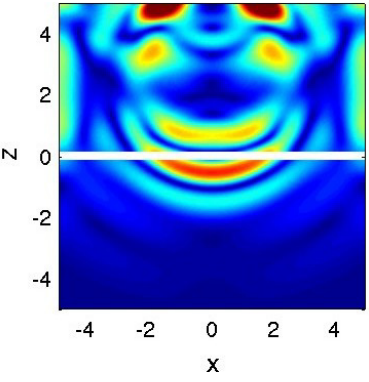

(b) $\left|\widetilde{\mathbf{u}}_{\eta}(x, 0, z, 5)\right|$

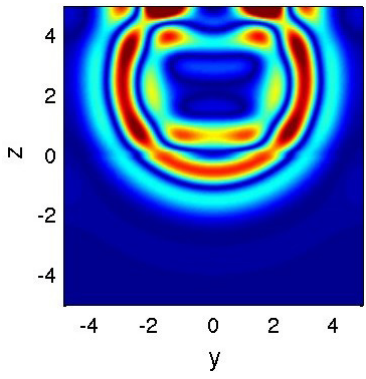

(c) $\left|\mathbf{u}_{\eta}(0, y, z, 5)\right|$

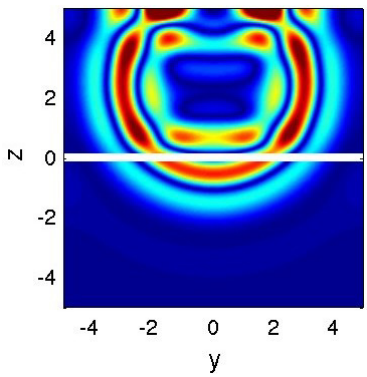

(d) $\left|\widetilde{\mathbf{u}}_{\eta}(0, y, z, 5)\right|$

Figure 8. 3-D case: exact (a,c) and effective (b,d) transmission solutions for $\eta=0.2$ in coordinate planes $y=0(\mathrm{a}, \mathrm{b})$ and $x=0(\mathrm{c}, \mathrm{d})$, evaluated at $t=5$.

\subsubsection{3-D numerical experiments}

3 -D results are given with the computational domain again defined by $L=5$. The material parameters are set to $\left(\rho^{-}, \mu^{-}, \lambda^{-}\right)=(1.8,1.6,2.2)$ and $\left(\rho^{+}, \mu^{+}, \lambda^{+}\right)=(1,1,1)$ outside the layer, and $\left(\rho^{\mathrm{I}}, \lambda^{\mathrm{I}}, \mu^{\mathrm{I}}\right)=(2,4,3)$ inside the layer. The source is again of the form (5.23), with $\mathbf{x}_{0}=(0,0,2.5)$ and $f_{0}=0.5$. The mesh (Fig. $\left.4 \mathrm{~b}\right)$ is made of cubic elements of size $h=1.0$ and of order 6 . The time discretization uses the semi-implicit scheme (5.13), with the time step set to $\Delta t=0.0002$. The exact and effective transmission solutions for $\eta=0.2$ are shown (in terms of respective horizontal displacements at $t=5$ ) on Figure 8. Then, computing the $L^{2}$ relative error $(5.24)$ at $t=5$ for different values of $\eta$, Figure 9 shows that again $E(\eta)=O\left(\eta^{3}\right)$, consistently with Theorem 2.5.

\section{Conclusion And OUtLOOK}

In this paper we have developed the basic tools for a treatment of thin interfaces via effective transmission conditions for isotropic elastodynamics, their theoretical justification, and their numerical treatment. As it stands here, the work is complete for the rather academic case of a homogeneous and planar interface with constant thickness. However, many useful extensions can be considered.

First, the treatment of (smooth) curved layers is of interest. The only (albeit substantial) additional difficulties are purely technical and related to the use of tools of differential geometry. 


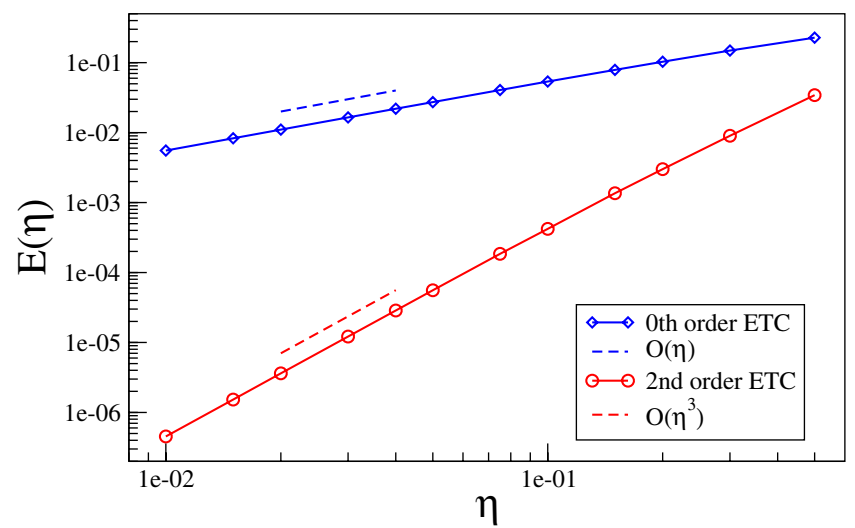

Figure 9. 3-D case: relative $L^{2}$ difference $E(\eta ; 5)$ as a function of $\eta$ for (i) the second-order ETC model and (ii) the model where the layer is ignored.

A rather direct extension of this article is the treatment of layered interfaces, where the material properties inside the layer are no longer constant but of the form

$$
\lambda_{\eta}^{\mathrm{I}}(\mathbf{y}, \nu)=\lambda^{\mathrm{I}}(\nu / \eta), \quad \mu_{\eta}^{\mathrm{I}}(\mathbf{y}, \nu)=\mu^{\mathrm{I}}(\nu / \eta), \quad \rho_{\eta}^{\mathrm{I}}(\mathbf{y}, \nu)=\rho^{\mathrm{I}}(\nu / \eta),
$$

where $\lambda^{\mathrm{I}}(s), \mu^{\mathrm{I}}(s), \rho^{\mathrm{I}}(s)$ are reference (non necessarily smooth) functions defined for $-\frac{1}{2}<s<\frac{1}{2}$, i.e. in the normalized layer. In the same spirit, it should be easy to incorporate smooth lateral variations of the the layer constitutive parameters through

$$
\lambda_{\eta}^{\mathrm{I}}(\mathbf{y}, \nu)=\lambda^{\mathrm{I}}(\mathbf{y}, \nu / \eta), \quad \mu_{\eta}^{\mathrm{I}}(\mathbf{y}, \nu)=\mu^{\mathrm{I}}(\mathbf{y}, \nu / \eta), \quad \rho_{\eta}^{\mathrm{I}}(\mathbf{y}, \nu)=\rho^{\mathrm{I}}(\mathbf{y}, \nu / \eta),
$$

where $\lambda^{\mathrm{I}}(\mathbf{y}, s), \mu^{\mathrm{I}}(\mathbf{y}, s), \rho^{\mathrm{I}}(\mathbf{y}, s)$ now are reference functions defined in the normalized layer $\Omega_{1}^{\mathrm{I}}$ and smooth with respect to the lateral coordinate $\mathbf{y}$.

The case of a layer of non-constant thickness, of the form $\Omega_{\eta}^{\mathrm{E}}:=\left\{(\mathbf{y}, \nu),-\eta f_{-}(\mathbf{y}) \leq \nu \leq \eta f_{+}(\mathbf{y})\right\}$ (where $f_{ \pm}$are smooth positive functions), is trickier but amenable to an approach similar to that developed here.

Once available, formulations for the above three cases treated separately would provide natural ingredients towards the treatment of curved interfaces with non-uniform thickness and constitutive properties (with smooth lateral variations of the geometry and coefficients). On the other hand, the treatment of non-smooth and / or singular lateral variations of the geometry and coefficients requires new ideas and techniques.

Another challenging topic is the derivation of higher-order equivalent transmission conditions, even for the present case of a homogeneous and planar layer with constant thickness. As already mentioned in Remark 3.6, such a derivation should exploit the asymptotic expansion of $\mathbf{u}_{\eta}$ obtained in this paper, but we anticipate some difficulties in the construction of stable higher-order ETCs.

\section{APPENDIX A. INTERIOR ASYMPTOTIC EXPANSION}

The sequence of Dirichlet problem $(3.12 \mathrm{a}, \mathrm{b})$ may be recast in the form

$$
\partial_{s}^{2} \mathbf{U}^{\ell}(s)=\mathbf{F}^{\ell}(s) \quad\left(-\frac{1}{2} \leq s \leq \frac{1}{2}\right) \quad \text { with }\left\{\mathbf{U}^{\ell}(s)\right\}_{1}=\left\{\mathbf{u}^{\ell}(s)\right\},
$$

(where $\left\{\mathbf{u}^{\ell}(s)\right\}$ denotes an arbitrary Dirichlet data expressed in generalized jump form), having set

$$
\mathbf{F}^{\ell}(s)=\mathcal{Q}_{1} \partial_{s} \mathbf{U}^{\ell-1}(s)+\mathcal{Q}_{2} \mathbf{U}^{\ell-2}(s), \quad \text { with } \mathcal{Q}_{1}:=-A^{-1} \mathcal{P}_{1}, \mathcal{Q}_{2}:=A^{-1}\left(\rho^{\mathrm{I}} \partial_{t}^{2}-\mathcal{P}_{2}\right)
$$


The solution to any problem of the form (A.1a) is given by

$$
\mathbf{U}^{\ell}(s)=\left\langle\mathbf{u}^{\ell}\right\rangle+\left[\mathbf{u}^{\ell}\right] s+S \mathbf{F}^{\ell}(s), \quad S \mathbf{F}(s):=\int_{-1 / 2}^{s}\left(s-\frac{1}{2}\right)\left(t+\frac{1}{2}\right) \mathbf{F}(t) \mathrm{d} t+\int_{s}^{1 / 2}\left(s+\frac{1}{2}\right)\left(t-\frac{1}{2}\right) \mathbf{F}(t) \mathrm{d} t
$$

where $\mathbf{V}(s)=S \mathbf{F}(s)$ is, by construction, the solution of $\partial_{s}^{2} \mathbf{V}(s)=\mathbf{F}(s), \mathbf{V}\left( \pm \frac{1}{2}\right)=0$.

We now prove Lemma 3.3 by induction. We first note that, using (3.18a), equation (3.17) with $\ell=0,1$ agrees with expressions (3.13) of $\mathbf{U}^{0}$ and (3.14) of $\mathbf{U}^{1}$. Now, assume that $\mathbf{U}^{\ell-2}$ and $\mathbf{U}^{\ell-1}$ are of the form (3.17) with (3.18a). Using(3.17) in $\mathbf{F}^{\ell}$ defined by (A.1b), one finds

$$
\begin{aligned}
\mathbf{F}^{\ell}(s) & =\mathcal{Q}_{1}\left\{\sum_{j=0}^{\ell-1} \partial_{s} \mathcal{X}_{j}(s)\left\langle\mathbf{u}^{\ell-j-1}\right\rangle+\partial_{s} \mathcal{Y}_{j-1}(s)\left[\mathbf{u}^{\ell-j}\right]\right\}+\mathcal{Q}_{2}\left\{\sum_{j=0}^{\ell-2} \mathcal{X}_{j}(s)\left\langle\mathbf{u}^{\ell-j-2}\right\rangle+\mathcal{Y}_{j-1}(s)\left[\mathbf{u}^{\ell-j-1}\right]\right\} \\
& =\sum_{j=0}^{\ell-2}\left\{\left(\mathcal{Q}_{1} \partial_{s} \mathcal{X}_{j+1}(s)+\mathcal{Q}_{2} \mathcal{X}_{j}(s)\right)\left\langle\mathbf{u}^{\ell-j-2}\right\rangle+\left(\mathcal{Q}_{1} \partial_{s} \mathcal{Y}_{j}(s)+\mathcal{Q}_{2} \mathcal{Y}_{j-1}(s)\right)\left[\mathbf{u}^{\ell-j-1}\right]\right\}
\end{aligned}
$$

where the last equality result from shifting the summation index by one and taking advantage of (3.18a). Invoking (A.2) and shifting the summation index, the solution $\mathbf{U}^{\ell}$ of $(\mathrm{A} .1 \mathrm{a}, \mathrm{b})$ is then obtained as

$\mathbf{U}^{\ell}(s)=\left\langle\mathbf{u}^{\ell}\right\rangle+\left[\mathbf{u}^{\ell}\right] s+\sum_{j=2}^{\ell}\left\{S\left(\mathcal{Q}_{1} \partial_{s} \mathcal{X}_{j-1}(s)+\mathcal{Q}_{2} \mathcal{X}_{j-2}(s)\right)\left\langle\mathbf{u}^{\ell-j}\right\rangle+S\left(\mathcal{Q}_{1} \partial_{s} \mathcal{Y}_{j-2}(s)+\mathcal{Q}_{2} \mathcal{Y}_{j-3}(s)\right)\left[\mathbf{u}^{\ell-j+1}\right]\right\}$.

It is therefore of the form (3.17), provided the tangential operators $\mathcal{X}_{j}$ and $\mathcal{Y}_{j}$ are linked by the recurrence relations

$$
\mathcal{X}_{j}(s)=S\left(\mathcal{Q}_{1} \partial_{s} \mathcal{X}_{j-1}(s)+\mathcal{Q}_{2} \mathcal{X}_{j-2}(s)\right), \quad \mathcal{Y}_{j-1}(s)=S\left(\mathcal{Q}_{1} \partial_{s} \mathcal{Y}_{j-2}(s)+\mathcal{Q}_{2} \mathcal{Y}_{j-3}(s)\right) \quad(j \geq 2)
$$

which, with the help of initialization (3.18a), recursively determine all $\mathcal{X}_{j}$ and $\mathcal{Y}_{j}$. In particular, setting $f_{0}(s)=1$, $f_{1}(s)=s, f_{2}(s)=s^{2}$, one finds

$$
\left[S f_{0}\right](s)=\frac{s^{2}}{2}-\frac{1}{8}, \quad\left[S f_{1}\right](s)=\frac{s^{3}}{6}-\frac{s}{24}, \quad\left[S f_{2}\right](s)=\frac{s^{4}}{12}-\frac{1}{192} .
$$

The first few operators $\mathcal{X}_{j}$ and $\mathcal{Y}_{j}$ are then easily found to be given by

$$
\begin{aligned}
& \mathcal{X}_{2}(s)=\left(\frac{s^{2}}{2}-\frac{1}{8}\right) \mathcal{Q}_{2}, \quad \mathcal{X}_{3}(s)=\left(\frac{s^{3}}{6}-\frac{s}{24}\right) \mathcal{Q}_{1} \mathcal{Q}_{2}, \\
& \mathcal{X}_{4}(s)=\left(\frac{s^{4}}{24}-\frac{1}{384}\right)\left(\mathcal{Q}_{1} \mathcal{Q}_{1}+\mathcal{Q}_{2}\right) \mathcal{Q}_{2}-\left(\frac{s^{2}}{48}-\frac{1}{192}\right)\left(\mathcal{Q}_{1} \mathcal{Q}_{1}+3 \mathcal{Q}_{2}\right) \mathcal{Q}_{2},
\end{aligned}
$$




$$
\begin{aligned}
& \mathcal{Y}_{1}(s)=\left(\frac{s^{2}}{2}-\frac{1}{8}\right) \mathcal{Q}_{1}, \quad \mathcal{Y}_{2}(s)=\left(\frac{s^{3}}{6}-\frac{s}{24}\right)\left[\mathcal{Q}_{1} \mathcal{Q}_{1}+\mathcal{Q}_{2}\right] \\
& \mathcal{Y}_{3}(s)=\left(\frac{s^{4}}{24}-\frac{1}{384}\right)\left(\mathcal{Q}_{1} \mathcal{Q}_{1} \mathcal{Q}_{1}+\mathcal{Q}_{2} \mathcal{Q}_{1}+\mathcal{Q}_{1} \mathcal{Q}_{2}\right)-\left(\frac{s^{2}}{48}-\frac{1}{192}\right)\left(\mathcal{Q}_{1} \mathcal{Q}_{1} \mathcal{Q}_{1}+\mathcal{Q}_{1} \mathcal{Q}_{2}+3 \mathcal{Q}_{2} \mathcal{Q}_{1}\right) .
\end{aligned}
$$

Finally, the boundary values $\mathcal{X}_{j}\left( \pm \frac{1}{2}\right)=\mathcal{Y}_{j}\left( \pm \frac{1}{2}\right)=0(j \geq 2)$ result directly from relations (A.3) and the definition (A.2) of $S$, while the polynomial nature in $s$ and the parity properties (3.18c) are easily established by straightforward induction on $j$ based on (A.2) and relations (3.18b).

\section{REFERENCES}

[1] H. Ammari and C. Latiri-Grouz, Conditions aux limites approchées pour les couches minces périodiques. ESAIM: M2AN 33 (1999) 673-692.

[2] X. Antoine and H. Barucq, Approximation by generalized impedance boundary conditions of a transmission problem in acoustic scattering. ESAIM: M2AN 39 (2005) 1041-1059.

[3] A. Bendali and K. Lemrabet, The effect of a thin coating on the scattering of a time-harmonic wave for the helmholtz equation. SIAM J. Appl. Math. 56 (1996) 1664-1693.

[4] I. Bihari, A generalization of a lemma of bellman and its application to uniqueness problems of differential equations. Acta Math. Hungarica 7 (1956) 81-94.

[5] S. Chun, H. Haddar J.S. Hesthaven, High-order accurate thin layer approximations for time-domain electromagnetics, part ii: transmission layers. J. Comput. Appl. Math. 234 (2010) 2587-2608.

[6] G Cohen, Higher-order numerical methods for transient wave equations. Springer-Verlag (2001).

[7] M. Dauge, S. Tordeux and G. Vial, Self-similar perturbation near a corner: matching versus multiscale expansions for a model problem. Around the Research of Vladimir Maz'ya II, Partial Differential Equations. Vol. 12 of International Mathematical Series. Springer (2010) 95-134.

[8] B. Delourme, H. Haddar and P. Joly, Approximate models for wave propagation across thin periodic interfaces. J. Math. pures Appl. 98 (2012) 28-71.

[9] B. Engquist and A. Majda, Absorbing boundary conditions for the numerical simulation of waves. Math. Comput. 31 (1977) 629-651.

[10] B. Engquist and J.-C. Nédélec, Effective boundary conditions for acoustic and electromagnetic scattering in thin layers. Technical report, Technical Report of CMAP, 278 (1993).

[11] L.C. Evans, Partial differential equations. American Mathematical Society (1998).

[12] G. Geymonat, S. Hendili, F. Krasucki and M. Vidrascu, Matched asymptotic expansion method for a homogenized interface model. 24 (2014) 573-597.

[13] D. Gilbarg and N.S. Trudinger, Elliptic partial differential equations of second order. Springer-Verlag (2001).

[14] H. Haddar and P. Joly, Stability of thin layer approximation of electromagnetic waves scattering by linear and non linear coatings. Stud. Math. Appl. 31 (2002) 415-456.

[15] H Haddar and P. Joly, Stability of thin layer approximation of electromagnetic waves scattering by linear and nonlinear coatings. J. Comput. Appl. Math. 143 (2002) 201-236.

[16] H. Haddar, P. Joly and H.-M. Nguyen, Generalized impedance boundary conditions for scattering problems from strongly absorbing obstacles: the case of Maxwell's equations. Math. Models Methods Appl. Sci. 18 (2008) 1787-1827.

[17] P. Joly, Analyse et approximation de modèles de propagation d'ondes. analyse mathématique. Lecture notes, Ecole polytechnique, Palaiseau, France (2002).

[18] V. Maz'ya, S.A. Nazarov and B.A. Plamenevskii, Asymptotic theory of elliptic boundary value problems under a singular perturbation of the domains, vols. 1 and 2. Birkhaüser (2000).

[19] W. McLean, Strongly elliptic systems and boundary integral equations. Cambridge (2000).

[20] V. Péron, Equivalent boundary conditions for an elasto-acoustic problem set in a domain with a thin layer. ESAIM: M2AN 48 (2014) 1431-1449.

[21] K. Schmidt and A. Chernov, A unified analysis of transmission conditions for thin conducting sheets in the time-harmonic eddy current model. SIAM J. Appl. Math. 73 (2013) 1980-2003.

[22] T.B.A. Senior and J.L. Volakis, Generalized impedance boundary conditions in scattering. Proc. IEEE 79 (1991) 1413-1420.

[23] T.B.A Senior and J.L. Volakis, Approximate boundary conditions in electromagnetics. Institution of Electrical Engineers, London, UK (1995).

[24] L.N. Trefethen and L. Halpern, Well-posedness of one-way wave equations and absorbing boundary conditions. Math. Comput. 47 (1986) 421-435. 